\title{
Pointwise Green's Function Estimates Toward Stability for Degenerate Viscous Shock Waves
}

\author{
PETER HOWARD
}

Department of Mathematics, Texas A \& M University, College Station, Texas, USA

\begin{abstract}
We consider degenerate viscous shock waves arising in systems of two conservation laws, where degeneracy describes viscous shock waves for which the asymptotic endstates are sonic to the hyperbolic system (the shock speed is equal to one of the characteristic speeds). In particular, we develop detailed pointwise estimates on the Green's function associated with the linearized perturbation equation, sufficient for establishing that spectral stability implies nonlinear stability. The analysis of degenerate viscous shock waves involves several new features, such as algebraic (nonintegrable) convection coefficients, loss of analyticity of the Evans function at the leading eigenvalue, and asymptotic time decay of perturbations intermediate between that of the Lax case and that of the undercompressive case.
\end{abstract}

Keywords Conservation laws; Degenerate shock waves; Stability.

Mathematics Subject Classification 35L65; 35K 55.

\section{Introduction}

We consider degenerate viscous shock waves arising in the system:

$$
\begin{gathered}
u_{t}+f(u)_{x}=u_{x x}, \quad u, f \in \mathbb{R}^{2}, \\
u(0, x)=u_{0}(x)
\end{gathered}
$$

that is, solutions of the form $\bar{u}(x-s t)=\left(\bar{u}_{1}(x-s t), \bar{u}_{2}(x-s t)\right)^{\operatorname{tr}}, \quad \bar{u}( \pm \infty)=$ $\left(u_{1}^{ \pm}, u_{2}^{ \pm}\right)^{\operatorname{tr}}$, that satisfy the Rankine-Hugoniot condition

$$
s=\frac{f_{k}\left(u_{1}^{+}, u_{2}^{+}\right)-f_{k}\left(u_{1}^{-}, u_{2}^{-}\right)}{u_{k}^{+}-u_{k}^{-}}, \quad k=1,2
$$

Received April 1, 2004; Accepted August 17, 2005

Address correspondence to Peter Howard, Department of Mathematics, Texas A \& M University, College Station, TX 77843, USA; E-mail: phoward@math.tamu.edu 
and for which $s \in \operatorname{Spectrum}\left(d f\left(u_{ \pm}\right)\right)=\left\{a_{k}^{ \pm}\right\}_{k=1}^{2}$. Throughout the analysis, we will make the following assumptions on the structure of (1.1) and the wave $\bar{u}$ :

(H0) $f \in C^{2}(\mathbb{R})$;

(H1) (Lax degeneracy) Either $a_{1}^{-}<s<a_{2}^{-}$and $a_{1}^{+}<s=a_{2}^{+}$(right side degenerate) or $a_{1}^{-}=s<a_{2}^{-}$and $a_{1}^{+}<s<a_{2}^{+}$(left side degenerate);

(H2) (First order degeneracy) For either case $a_{k}^{ \pm}=s$ (right or left side degeneracy), we assume there holds

$$
l_{k}^{ \pm} d^{2} f\left(u_{ \pm}\right)\left(r_{k}^{ \pm}, r_{k}^{ \pm}\right) \neq 0
$$

where $l_{k}^{ \pm}$and $r_{k}^{ \pm}$denote the left and right eigenvectors of $d f\left(u_{ \pm}\right)$respectively, and $d^{2} f\left(u_{ \pm}\right)$denotes the operator

$$
\begin{aligned}
& d^{2} f\left(u_{ \pm}\right)(v, v) \\
& \quad=\left(\begin{array}{l}
\frac{1}{2} \partial_{u_{1} u_{1}} f_{1}\left(u_{1}^{ \pm}, u_{2}^{ \pm}\right) v_{1}^{2}+\partial_{u_{1} u_{2}} f_{1}\left(u_{1}^{ \pm}, u_{2}^{ \pm}\right) v_{1} v_{2}+\frac{1}{2} \partial_{u_{2} u_{2}} f_{1}\left(u_{1}^{ \pm}, u_{2}^{ \pm}\right) v_{2}^{2} \\
\frac{1}{2} \partial_{u_{1} u_{1}} f_{2}\left(u_{1}^{ \pm}, u_{2}^{ \pm}\right) v_{1}^{2}+\partial_{u_{1} u_{2}} f_{2}\left(u_{1}^{ \pm}, u_{2}^{ \pm}\right) v_{1} v_{2}+\frac{1}{2} \partial_{u_{2} u_{2}} f_{2}\left(u_{1}^{ \pm}, u_{2}^{ \pm}\right) v_{2}^{2}
\end{array}\right)
\end{aligned}
$$

Under assumption (H2), both $\bar{u}_{1}(x-s t)$ and $\bar{u}_{2}(x-s t)$ decay to the degenerate side endstate with rate $|x-s t|^{-1}$ (see Howard and Zumbrun, 2004). Though (H1)-(H2) are stated for clarity in terms of an arbitrary wave speed $s$, the generality of $f$ allows us to take $s=0$ without loss of generality by shifting to a moving coordinate frame $x \mapsto x-s t$ and replacing $f$ with $f-s u$, which continues to satisfy our only general requirement $(\mathrm{H} 0)$. Throughout the article, then, we will consider a standing wave $\bar{u}(x)$.

We note that assumptions (H1) and (H2) describe the most generic degenerate case. In particular, (H1) asserts that there is only degeneracy on one side, and not associated with both characteristics, while (H2) is analogous to the condition for single equations $f^{\prime \prime}\left(u_{ \pm}\right) \neq 0$ (see Howard, 2002a,b). Finally, we mention that our restriction to the case of identity viscosity and two equations follows from technical restrictions in the Evans function analysis of Howard and Zumbrun (2004), and that we regard the cases of general viscosity (including partial regularization) and an arbitrary number of equations as interesting directions for future work.

Under the assumption of conditions ( $\mathrm{H} 0)-(\mathrm{H} 2)$, we develop detailed estimates on the Green's function of the linearized perturbation equation sufficient for establishing that spectral stability implies nonlinear stability for degenerate viscous shock waves arising in (1.1). The full development of nonlinear stability is carried out in a companion article, (Howard, 2005). We remark that the only systems analysis to date in the case of degenerate viscous shock profiles regards the partially regularized $p$-system,

$$
\begin{gathered}
v_{t}-u_{x}=0 \\
u_{t}-p(v)_{x}=u_{x x},
\end{gathered}
$$

which can be reduced through coupling to a scalar analysis and analyzed by energy methods (see Nishihara, 1995).

Our interest in the degenerate case is motivated both by the physical application to detonation waves (see Howard, 2002a and the references therein) and by 
its position as a boundary case between Lax and undercompressive waves. The critical new feature in the analysis of degenerate waves, necessarily absent in the case of nondegenerate waves (for which $s \notin \operatorname{Spectrum}\left(d f\left(u_{ \pm}\right)\right)$), is the algebraic (nonintegrable) decay to endstate of the degenerate wave (see hypothosis $(\mathrm{H} 2)$ ). As a consequence of this slow decay, the convection coefficients for the perturbation equation found by linearizing (1.1) about $\bar{u}(x)$ are also nonintegrable, and the consequent asymptotic analysis is considerably more complicated than that of the nondegenerate case. More important, the Evans function associated with the linearized operator has terms of the form $\sqrt{\lambda} \ln \lambda$, and hence analyticity is lost at the critical point $\lambda=0$, around which long time behavior is determined. This loss of analyticity has two critical consequences: (1) the study of the point spectrum of the linear operator near $\lambda=0$ is more delicate; and (2) the time decay of the linear semigroup operator $e^{L t}$ is reduced. While the former of these has been considered in Howard and Zumbrun (2004) - in the case of conservation laws-and in Sandstede and Scheel (2004) - in the case of reaction-diffusion systems-the latter has not (to our knowledge) been addressed. This, then, is the critical issue of the current analysis.

It is well known that solutions $u(t, x)$ of $(1.1)$, initialized by $u(0, x)$ near a standing wave solution $\bar{u}(x)$, will not generally approach $\bar{u}(x)$, but rather will approach a translate of $\bar{u}(x)$ determined by the amount of mass (measured by $\left.\int_{\mathbb{R}}(u(0, x)-\bar{u}(x)) d x\right)$ carried into the shock as well as the amount carried out to the far field along outgoing characteristics. In our framework, a local tracking function $\delta(t)$ will serve to approximate the shift of this translate at each time $t$. Following Howard and Zumbrun (2000), we build this shift into our model by defining our perturbation $v(t, x)$ as $v(t, x):=u(t, x+\delta(t))-\bar{u}(x)$. We will say that $\bar{u}(x)$ is (orbitally) stable with respect to some measure if for $v(0, x)$ sufficiently small in that measure we have $v(t, x) \rightarrow 0$ as $t \rightarrow \infty$.

Substituting $v(t, x)=u(t, x+\delta(t))-\bar{u}(x)$ into (1.1), we obtain the perturbation equation

$$
v_{t}=L v+Q(v)_{x}+\dot{\delta}(t)\left(\bar{u}_{x}(x)+v_{x}\right)
$$

where $L v:=v_{x x}-(A(x) v)_{x}, \quad A(x)=d f(\bar{u}(x))$, and $Q(v)=\mathbf{O}\left(v^{2}\right)$ is a smooth function of $v$. Restricting the discussion without loss of generality to the case of right-side degeneracy (see (H1)), we observe that according to hypotheses (H0)-(H2), we have, for $A_{ \pm}:=\lim _{x \rightarrow \pm \infty} A(x)$, that $A(x) \in C^{1}(\mathbb{R})$ and

$$
\begin{aligned}
& \left|\partial_{x}^{k}\left(A(x)-A_{+}\right)\right|=\mathbf{O}\left(|x|^{-k-1}\right), \quad k=0,1,(\text { degenerate side }) \\
& \left|\partial_{x}^{k}\left(A(x)-A_{-}\right)\right|=\mathbf{O}\left(e^{-\alpha|x|}\right), \quad k=0,1, \text { (nondegenerate side) }
\end{aligned}
$$

for some $\alpha>0$.

Integrating (1.2), we have (after integration by parts on the second integral and observing that $\left.e^{L t} \bar{u}_{x}(x)=\bar{u}_{x}(x)\right)$,

$$
\begin{aligned}
v(t, x)= & \int_{-\infty}^{+\infty} G(t, x ; y) v_{0}(y) d y+\delta(t) \bar{u}_{x}(x) \\
& -\int_{0}^{t} \int_{-\infty}^{+\infty} G_{y}(t-s, x ; y)[Q(v(s, y))+\dot{\delta}(s) v(s, y)] d y d s,
\end{aligned}
$$


where $G(t, x ; y)$ represents a Green's function for the linear part of (1.2):

$$
G_{t}+(A(x) G)_{x}=G_{x x} ; \quad G(0, x ; y)=\delta_{y}(x) I .
$$

The goal of the current analysis is to develop pointwise estimates on $G(t, x ; y)$ sufficient for establishing that an iteration on $v(t, x)$ will close. The estimates on $G(t, x ; y)$ will be divided into those terms for which the $x$ dependence is exactly $\bar{u}_{x}(x)$ (referred to as the excited terms, and denoted $E(t, x ; y)=\bar{u}_{x}(x) e(t, y)$ ) and those for which the $x$ dependence is not exactly $\bar{u}_{x}(x)$. Typically, the excited terms do not decay in $t$, and represent mass that accumlates in the shock layer, shifting the shock. Our approach will be to choose our shift $\delta(t)$ to annihilate this mass, so that we track the shock in time. Following Howard and Zumbrun (2000), we write

$$
G(t, x ; y)=\widetilde{G}(t, x ; y)+\bar{u}_{x}(x) e(t, y),
$$

for which we have

$$
\begin{aligned}
v(t, x)= & \int_{-\infty}^{+\infty} \widetilde{G}(t, x ; y) v_{0}(y) d y+\bar{u}_{x}(x) \int_{-\infty}^{+\infty} e(t, y) v_{0}(y) d y+\delta(t) \bar{u}_{x}(x) \\
& -\int_{0}^{t} \int_{-\infty}^{+\infty} G_{y}(t-s, x ; y)[Q(v(s, y))+\dot{\delta}(s) v(s, y)] d y d s .
\end{aligned}
$$

Choosing $\delta(t)$ to eliminate the linear excited terms, we have

$$
\delta(t):=-\int_{-\infty}^{+\infty} e(t, y) v_{0}(y) d y
$$

and consequently

$v(t, x)=\int_{-\infty}^{+\infty} \widetilde{G}(t, x ; y) v_{0}(y) d y-\int_{0}^{t} \int_{-\infty}^{+\infty} G_{y}(t-s, x ; y)[Q(v(s, y))+\dot{\delta}(s) v(s, y)] d y d s$.

We mention that in the iteration on $v(t, x)$, we only require an estimate on $\dot{\delta}(t)$, so in practice we consider the time derivative of (1.4).

Typically, we analyze $G(t, x ; y)$ through its Laplace transform, $G_{\lambda}(x, y)$, which satisfies the $\operatorname{ODE}(t \rightarrow \lambda)$

$$
G_{\lambda_{x x}}-\left(A(x) G_{\lambda}\right)_{x}-\lambda G_{\lambda}=-\delta_{y}(x) I
$$

and can be estimated by standard methods. Letting $\varphi_{1}^{+}$and $\varphi_{2}^{+}$represent the (necessarily) two linearly independent asymptotically decaying solutions at $+\infty$ of the eigenvalue ODE

$$
L \varphi=\lambda \varphi
$$

( $L$ as in (1.2)) and $\varphi_{1}^{-}$and $\varphi_{2}^{-}$similarly the two linearly independent asymptotically decaying solutions at $-\infty$, we write $G_{\lambda}(x, y)$ as a linear combination,

$$
G_{\lambda}(x, y)= \begin{cases}\varphi_{1}^{+}(x) N_{1}^{-}(y)+\varphi_{2}^{+}(x) N_{2}^{-}(y), & x>y \\ \varphi_{1}^{-}(x) N_{1}^{+}(y)+\varphi_{2}^{-}(x) N_{2}^{+}(y), & x<y\end{cases}
$$


where we observe the notation

$$
\varphi_{k}^{ \pm} N_{k}^{\mp}=\left(\begin{array}{c}
\varphi_{k 1}^{ \pm} \\
\varphi_{k 2}^{ \pm}
\end{array}\right)\left(\begin{array}{ll}
N_{k 1}^{\mp} & N_{k 2}^{\mp}
\end{array}\right)=\left(\begin{array}{cc}
\varphi_{k 1}^{ \pm} N_{k 1}^{\mp} & \varphi_{k 1}^{ \pm} N_{k 2}^{\mp} \\
\varphi_{k 2}^{ \pm} N_{k 1}^{\mp} & \varphi_{k 2}^{ \pm} N_{k 2}^{\mp}
\end{array}\right) .
$$

Insisting on the continuity of $G_{\lambda}(x, y)$ across $y=x$ and a step in $\partial_{x} G_{\lambda}(x, y)$ (leading to the Dirac delta), we have

$$
\begin{gathered}
\left(\varphi_{1}^{+} N_{1}^{-}+\varphi_{2}^{+} N_{2}^{-}-\varphi_{1}^{-} N_{1}^{+}-\varphi_{2}^{-} N_{2}^{+}\right)=0 \\
\left(\varphi_{1}^{+^{\prime}} N_{1}^{-}+\varphi_{2}^{+^{\prime}} N_{2}^{-}-\varphi_{1}^{-\prime} N_{1}^{+}-\varphi_{2}^{-\prime} N_{2}^{+}\right)=-I .
\end{gathered}
$$

Equations (1.6) represent eight equations and eight unknowns, which decouple into two sets of four equations and four unknowns. Solving by Cramer's formula, we have, for example,

$$
N_{11}^{-}(y ; \lambda)=-\frac{\operatorname{det}\left(\begin{array}{ccc}
\varphi_{21}^{+} & \varphi_{11}^{-} & \varphi_{21}^{-} \\
\varphi_{22}^{+} & \varphi_{12}^{-} & \varphi_{22}^{-} \\
\varphi_{22}^{+^{\prime}} & \varphi_{12}^{-\prime} & \varphi_{22}^{-\prime}
\end{array}\right)}{\operatorname{det}\left(\begin{array}{llll}
\varphi_{1}^{+} & \varphi_{2}^{+} & \varphi_{1}^{-} & \varphi_{2}^{-} \\
\varphi_{1}^{+^{\prime}} & \varphi_{2}^{+^{\prime}} & \varphi_{1}^{-\prime^{\prime}} & \varphi_{2}^{-{ }^{\prime}}
\end{array}\right)}
$$

Clearly, then, $G_{\lambda}(x, y)$ will be well behaved so long as

$$
W(x ; \lambda):=\operatorname{det}\left(\begin{array}{llll}
\varphi_{1}^{+} & \varphi_{2}^{+} & \varphi_{1}^{-} & \varphi_{2}^{-} \\
\varphi_{1}^{+^{\prime}} & \varphi_{2}^{+^{\prime}} & \varphi_{1}^{-\prime^{\prime}} & \varphi_{2}^{-\prime}
\end{array}\right) \neq 0 .
$$

We define the Evans function as $D(\lambda):=W(0 ; \lambda)$, which in our setting is equivalent to the wedge-product formulations of Alexander et al. (1990), Evans (1972/1975), Gardner and Zumbrun (1998), Jones (1984), and Kapitula and Sandstede (1998). More precisely, in the wedge-product formulation, we denote

$$
\Phi_{k}^{ \pm}=\left(\begin{array}{c}
\varphi_{k}^{ \pm} \\
\varphi_{k}^{ \pm^{\prime}}
\end{array}\right)
$$

and set

$$
Y_{ \pm}(x ; \lambda)=\Phi_{1}^{ \pm}(x ; \lambda) \wedge \Phi_{2}^{ \pm}(x ; \lambda)
$$

where $\wedge$ denotes a general wedge product on the space of vectors in $\mathbb{R}^{4}$ (i.e., $\wedge$ denotes an associative, bilinear operation on vectors $v \in \mathbb{R}^{4}$ for which $v \wedge v=0$ ). In this case, we obtain through straightforward calculation that

$$
D(\lambda)=e^{-\int_{0}^{x} \operatorname{tr} \mathbb{M}(y ; \lambda) d y} Y_{-}(x ; \lambda) \wedge Y_{+}(x ; \lambda),
$$

which is the form considered in Alexander et al. (1990), Evans (1972/1975), Gardner and Zumbrun (1998), Jones (1984), and Kapitula and Sandstede (1998). (As described below, the matrix $\mathbb{M}$ is simply the matrix that arises when (1.5) 
is written as a first order system.) One advantage of (1.7) is that the two (sixcomponent) vectors $Y_{ \pm}$are the asymptotically decaying solutions of

$$
Y_{ \pm}^{\prime}=\mathscr{A}(x ; \lambda) Y_{ \pm}
$$

where $\mathscr{A}$ is defined by

$$
\begin{aligned}
Y_{ \pm}^{\prime} & =\left(\Phi_{1}^{ \pm} \wedge \Phi_{2}^{ \pm}\right)^{\prime}=\Phi_{1}^{ \pm^{\prime}} \wedge \Phi_{2}^{ \pm}+\Phi_{1}^{ \pm} \wedge \Phi_{2}^{ \pm^{\prime}} \\
& =\left(\mathbb{M} \Phi_{1}^{ \pm}\right) \wedge \Phi_{2}^{ \pm}+\Phi_{1}^{ \pm} \wedge \mathbb{M} \Phi_{2}^{ \pm}=\mathscr{A}_{ \pm} \Phi_{1}^{ \pm} \wedge \Phi_{2}^{ \pm} .
\end{aligned}
$$

In this way, the formulation (1.7) may remain valid in cases when our formulation is not. In the current setting, however, the vectors $\Phi_{k}^{ \pm}$can be distinctly defined, and our formulation is valid.

In order to understand the behavior of the Evans function, consider an eigenvector, $V(x ; \lambda)$, of the linear operator

$$
L v=v_{x x}-(A(x) v)_{x} .
$$

Since $V(x ; \lambda)$ must decay at both $\pm \infty$, it must be a linear combination of $\varphi_{1}^{+}$, and $\varphi_{2}^{+}$, and also of $\varphi_{1}^{-}$and $\varphi_{2}^{-}$. Consequently, these four solutions must be linearly dependent, and their Wronskian must be zero. In general, away from essential spectrum, zeros of the Evans function correspond with eigenvalues of the operator $L$, an observation that has been made precise in Alexander et al. (1990) in the case-pertaining to reaction diffusion equations-of isolated eigenvalues, and in Zumbrun and Howard (1998/2002), Gardner and Zumbrun (1998) in the casepertaining to conservation laws-of nonstandard "effective" eigenvalues embedded in essential spectrum of $L$. (The latter correspond with resonant poles of $L$, as examined in the scalar context in Pego and Weinstein, 1992.)

In Howard and Zumbrun (2004), the authors established that under assumptions $(\mathrm{H} 0)-(\mathrm{H} 2), D(\lambda)$ can be written as an analytic function plus a small error,

$$
D(\lambda)=D_{a}(\lambda)+\mathbf{O}\left(\left|\lambda^{3 / 2} \ln \lambda\right|\right), \quad \text { as } \lambda \rightarrow 0,
$$

where $D_{a}(\lambda)=\mathbf{O}(|\lambda|)$ is analytic in a neighborhood of $\lambda=0$. Following Howard and Zumbrun (2004), we introduce the following stability condition (D).

(D) : $D(\lambda)$ has precisely one zero in $\{\operatorname{Re} \lambda \geq 0\}$, necessarily at $\lambda=0$, and $D_{a}^{\prime}(0) \neq 0$.

While condition (D) is generally quite difficult to verify analytically (see, for example, Jones, 1984 in the context of the FitzHugh-Nagumo equations, and Dodd, 1996; Freistuhler and Szmolyan, 2002; Goodman, 1986; Humpherys and Zumbrun, 2002; Kawashima and Matsumura, 1985; Kawashima et al., 1986; Matsumura and Nishihara, 1985, and Plaza and Zumbrun, 2004 in the case of conservation laws), it can be checked numerically (see Brin, 2001; Oh and Zumbrun, 2003). A condition that lends itself more readily to exact study is the stability index, typically defined as

$$
\Gamma:=\operatorname{sgn} D_{a}^{\prime}(0) \times \operatorname{sgn} \lim _{\mathbb{R} \ni \lambda \rightarrow \infty} D(\lambda) .
$$


For $\lambda \in \mathbb{R}_{+}$, we have $D_{a}(\lambda) \in \mathbb{R}$, so that in the event that $\Gamma=-1, D(\lambda)$ must have a positive real root, which guarantees instability. In the case that $\Gamma=+1$, the question of stability remains undecided. It is shown, for example, in Howard and Zumbrun (2004) that the degenerate wave arising in the context of the fully regularized $p$-system

$$
\begin{aligned}
v_{t}-u_{x} & =v_{x x} \\
u_{t}-\left(v+v^{3}\right)_{x} & =u_{x x},
\end{aligned}
$$

satisfies $\Gamma=+1$.

Finally, we observe that if stability condition (D) holds, there exists a contour $\Gamma_{d}$ defined through

$$
\lambda_{d}(k)=-d_{0}+i d_{1} k-d_{2} k^{2}
$$

with $d_{0}$ and $d_{2}$ both positive constants, so that aside from the eigenvalue $\lambda=0$, the point spectrum of $L$ lies entirely to the left of $\Gamma_{d}$. We are now in a position to state the main result of the article.

Theorem 1.1. Suppose $\bar{u}(x)$ is a standing wave solution to (1.1) and suppose $(\mathrm{H} 0)-(\mathrm{H} 2)$ hold, as well as stability criterion (D). Then for some positive constants $M$, $K, P_{+}, \epsilon_{0}$ and $\eta$, depending only on $d f(\bar{u}(x))$ and the spectrum of $L$, the Green's function $G(t, x ; y)$ described through (1.3) satisfies the following estimates.

(i) $y \leq x \leq 0$ :

$$
\begin{aligned}
G(t, x ; y)= & \mathbf{O}\left(t^{-1 / 2}\right) e^{-\frac{\left(x-y-a_{2}^{-} t\right)^{2}}{M t}}+\mathbf{O}\left(t^{-1 / 2}\right) e^{\left.-\frac{\left(x-\frac{a_{1}^{-}}{a_{2}} y-a_{1}^{-} t\right.}{M t}\right)^{2}}+\bar{u}_{x}(x) e_{-}(t, y) \\
& +\mathbf{O}\left(\left(1+\left|x-\frac{a_{1}^{-}}{a_{2}^{-}} y-a_{1}^{-} t\right|+t^{1 / 2}\right)^{-3 / 2} \ln (e+t)\right) I_{\left\{\left|x-\frac{a_{1}^{-}}{a_{2}^{2}} y\right| \leq\left|a_{1}^{-}\right| t\right\}}, \\
G_{y}(t, x ; y)= & \mathbf{O}\left(t^{-1}\right) e^{-\frac{\left(x-y-a_{2}^{-} t\right)^{2}}{M t}}+\mathbf{O}\left(t^{-1}\right) e^{\left.-\frac{\left(x-\frac{a_{1}^{-}}{a_{2}} y-a_{1}^{-}\right.}{M t}\right)^{2}} \\
& +\mathbf{O}\left(\left(1+\left|x-\frac{a_{1}^{-}}{a_{2}^{-}} y-a_{1}^{-} t\right|+t^{1 / 2}\right)^{-5 / 2} \ln (e+t)\right) \partial_{y} e_{-}(t, y)
\end{aligned}
$$

where

$$
\begin{gathered}
e_{-}(t, y)=\mathbf{O}(1) e^{-\frac{\left(y+a_{2}^{-} t\right)^{2}}{M t}}+\mathbf{O}(1) I_{\left\{|y| \leq\left|a_{2}^{-}\right| t\right\}}, \\
\partial_{y} e_{-}(t, y)=\mathbf{O}\left(t^{-1 / 2}\right) e^{-\frac{\left(y+a_{2}^{-} t\right)^{2}}{M t}}+\mathbf{O}\left(\left(1+\left|y+a_{2}^{-} t\right|+t^{-1 / 2}\right)^{-3 / 2} \ln (e+t)\right) I_{\left\{|y| \leq\left|a_{2}^{-}\right| t\right\}}, \\
\partial_{t} e_{-}(t, y)=\mathbf{O}\left(t^{-1 / 2}\right) e^{-\frac{\left(y+a_{2}^{-} t\right)^{2}}{M t}}+\mathbf{O}\left(\left(1+\left|y+a_{2}^{-} t\right|+t^{-1 / 2}\right)^{-3 / 2} \ln (e+t)\right) I_{\left\{|y| \leq\left|a_{2}^{-}\right| t\right\}} ;
\end{gathered}
$$

(ii) $x \leq y \leq 0$ :

$$
\begin{aligned}
G(t, x ; y)= & \left.\mathbf{O}\left(t^{-1 / 2}\right) e^{-\frac{\left(x-y-a_{1}^{-} t\right)^{2}}{M t}}+\mathbf{O}\left(t^{-1 / 2}\right) e^{-\frac{\left(x-\frac{a_{1}^{-}}{a_{2}} y-a_{1}^{-} t\right.}{M t}}\right)^{2} \\
& +\mathbf{O}\left(\left(1+\left|x-\frac{a_{1}^{-}}{a_{2}^{-}} y-a_{1}^{-} t\right|+t^{1 / 2}\right)^{-3 / 2} \ln (e+t)\right) e_{-}(t, y) \\
& I_{\left\{\left|x-\frac{a_{1}^{-}}{a_{2}} y\right| \leq\left|a_{1}^{-}\right| t\right\}},
\end{aligned}
$$




$$
\begin{aligned}
G_{y}(t, x ; y)= & \mathbf{O}\left(t^{-1}\right) e^{-\frac{\left(x-y-a_{1}^{-} t\right)^{2}}{M t}}+\mathbf{O}\left(t^{-1}\right) e^{-\frac{\left(x-\frac{a_{1}^{-}}{a_{2}} y-a_{1}^{-} t\right.}{M t}}+\bar{u}_{x}(x) \partial_{y} e_{-}(t, y) \\
& +\mathbf{O}\left(\left(1+\left|x-\frac{a_{1}^{-}}{a_{2}^{-}} y-a_{1}^{-} t\right|+t^{1 / 2}\right)^{-5 / 2} \ln (e+t)\right) I_{\left\{\left|x-\frac{a_{1}^{-}}{a_{2}^{2}} y\right| \leq\left|a_{1}^{-}\right| t\right\}}
\end{aligned}
$$

(iii) $x \leq 0<K \leq y$ :

$$
\begin{aligned}
G(t, & ; y) \\
= & \mathbf{O}\left(t^{-1 / 2}\right) e^{-\frac{\left(x-a_{1}^{-} \int_{K}^{y} \frac{d s}{a_{1}(s)}-a_{1}^{-} t\right.}{M t}}+\bar{u}_{x}(x) e_{+}(t, y)+\mathbf{O}\left(t^{-1 / 2}\right) e^{-\frac{\left(x-a_{1}^{-} t\right)^{2}}{M t}} I_{\left\{\left|x-\int_{K}^{y} \frac{d s}{a_{1}(s)}\right| \leq\left|a_{1}^{-}\right| t\right\}} \\
& +\mathbf{O}\left(\left(1+\left|x-a_{1}^{-} \int_{K}^{y} \frac{d s}{a_{1}(s)}-a_{1}^{-} t\right|+t^{1 / 2}\right)^{-3 / 2} \ln (e+t)\right) I_{\left\{\left|x-a_{1}^{-} \int_{K}^{y} \frac{d s}{a_{1}(s)}\right| \leq\left|a_{1}^{-}\right| t\right\}} \\
& +\mathbf{O}\left(\left(1+\left|x-a_{1}^{-} t\right|+t^{1 / 2}\right)^{-3 / 2}\right) \mathbf{O}(1+|y|) I_{\left\{\left|x-\int_{K}^{y} \frac{d s}{a_{1}(s)}\right| \leq\left|a_{1}^{-}\right| t\right\}}, \\
G_{y}(t, x ; y) & \\
= & {\left[\mathbf{O}\left(t^{-1}(1+t)^{1 / 4}\right) \wedge \mathbf{O}\left(t^{-1}(1+|y|)\right)\right] e^{\left.-\frac{\left(x-a_{1}^{-} \int_{K}^{y} \frac{d s}{a_{1}(s)}-a_{1}^{-} t\right.}{M t}\right)^{2}}+\bar{u}_{x}(x) \partial_{y} e_{+}(t, y) } \\
& +\mathbf{O}\left(\left(1+\left|x-a_{1}^{-} \int_{K}^{y} \frac{d s}{a_{1}(s)}-a_{1}^{-} t\right|+t^{1 / 2}\right)^{-5 / 2} \ln (e+t)\right) I_{\left\{\left|x-a_{1}^{-} \int_{K}^{y} \frac{d s}{a_{1}(s)}\right| \leq\left|a_{1}^{-}\right| t\right\}} \\
& +\mathbf{O}\left(\left(1+\left|x-a_{1}^{-} t\right|+t^{1 / 2}\right)^{-3 / 2}\right) I_{\left\{\left|x-\int_{K}^{y} \frac{d s}{a_{1}(s)}\right| \leq\left|a_{1}^{-}\right| t\right\}},
\end{aligned}
$$

where

$$
\begin{aligned}
e_{+}(t, y)= & \mathbf{O}(1) e^{-\frac{\left(\int_{K}^{y} \frac{d s}{a_{1}(s)}+t\right)^{2}}{M t}}+\mathbf{O}(1) I_{\left\{\left|\int_{K}^{y} \frac{d s}{a_{1}(s)}\right| \leq t\right\}}+\mathbf{O}(1) e^{-\frac{y^{2}}{M t}}, \\
\partial_{y} e_{+}(t, y)= & \mathbf{O}\left(t^{-1 / 2}\right) e^{-\frac{\left(\int_{K}^{y} \frac{d s}{a_{1}(s)}+t\right)^{2}}{M t}}+\mathbf{O}\left(t^{-1 / 2}(1+t)^{-1 / 2}(1+|y|)\right) e^{-\frac{y^{2}}{M t}} \\
& +\left(\left(1+\left|\int_{K}^{y} \frac{d s}{a_{1}(s)}+t\right|+t^{1 / 2}\right)^{-3 / 2} \ln (e+t)\right) I_{\left\{\left|\int_{K}^{y} \frac{d s}{a_{1}(s)}\right| \leq t\right\}}, \\
\partial_{t} e_{+}(t, y)= & \mathbf{O}\left(t^{-1 / 2}\right) e^{-\frac{\left(\int_{K}^{y} \frac{d s}{a_{1}(s)}+t\right)^{2}}{M t}}+\mathbf{O}\left(t^{-1 / 2}(1+t)^{-1 / 2}\right) e^{-\frac{y^{2}}{M t}} \\
& +\left(\left(1+\left|\int_{K}^{y} \frac{d s}{a_{1}(s)}+t\right|+t^{1 / 2}\right)^{-3 / 2} \ln (e+t)\right) I_{\left\{\left|\int_{K}^{y} \frac{d s}{a_{1}(s)}\right| \leq t\right\}} ;
\end{aligned}
$$

(iv) $y \leq 0 \leq x$ :

$$
\begin{aligned}
G(t, x ; y)= & \mathbf{O}\left(t^{-1 / 2}(1+t)^{1 / 4}\right) \mathbf{O}\left((1+|x|)^{-1}\right) e^{-\frac{\left(x-y-a_{2}^{-} t\right)^{2}}{M t}} I_{\left\{|y| \geq a_{2}^{-} t\right\}}+\bar{u}_{x}(x) e_{-}(t, y) \\
& +\mathbf{O}\left(\left(1+\left|y+a_{2}^{-} t\right|\right)^{-1 / 2}\right) \mathbf{O}\left((1+|x|)^{-1}\right) e^{-\frac{x^{2}}{M t}} I_{\left\{|y| \leq a_{2}^{-} t\right\}}
\end{aligned}
$$




$$
\begin{aligned}
& +\mathbf{O}\left(t^{-1 / 2}(1+t)^{1 / 4} \ln (e+t)\right) \mathbf{O}\left((1+|x|)^{-2}\right) e^{-\frac{\left(x-y-a_{2}^{-} t\right)^{2}}{M t}} I_{\left\{|y| \geq a_{2}^{-} t\right\}} \\
& +\mathbf{O}\left(\left(1+\left|y+a_{2}^{-} t\right|\right)^{-1 / 2} \ln (e+t)\right) \mathbf{O}\left((1+|x|)^{-2}\right) e^{-\frac{x^{2}}{M t}} I_{\left\{|y| \leq a_{2}^{-} t\right\}}, \\
G_{y}(t, x ; y)= & \mathbf{O}\left(t^{-1}(1+t)^{1 / 4}\right) \mathbf{O}\left((1+|x|)^{-1}\right) e^{-\frac{\left(x-y-a_{2}^{-} t\right)^{2}}{M t}} I_{\left\{|y| \geq a_{2}^{-} t\right\}}+\bar{u}_{x}(x) \partial_{y} e_{-}(t, y) \\
& +\mathbf{O}\left(\left(1+\left|y+a_{2}^{-} t\right|\right)^{-3 / 2}\right) \mathbf{O}\left((1+|x|)^{-1}\right) e^{-\frac{x^{2}}{M t}} I_{\left\{|y| \leq a_{2}^{-} t\right\}} \\
& +\mathbf{O}\left(t^{-1}(1+t)^{1 / 4} \ln (e+t)\right) \mathbf{O}\left((1+|x|)^{-2}\right) e^{-\frac{\left(x-y-a_{2}^{-} t\right)^{2}}{M t}} I_{\left\{|y| \geq a_{2}^{-} t\right\}} \\
& +\mathbf{O}\left(\left(1+\left|y+a_{2}^{-} t\right|\right)^{-3 / 2} \ln (e+t)\right) \mathbf{O}\left((1+|x|)^{-2}\right) e^{-\frac{x^{2}}{M t}} I_{\left\{|y| \leq a_{2}^{-} t\right\}}
\end{aligned}
$$

(v) $0<K \leq y \leq x$ :

$$
\begin{aligned}
& G(t, x ; y) \\
& =\mathbf{O}\left(t^{-1 / 2}\right) \mathbf{O}\left((1+|x|)^{-1}\right) \mathbf{O}(1+|y|) e^{-\frac{(x-y)^{2}}{M t}}+P_{+} \bar{u}_{x}\left(I_{\left\{|x-y| \leq \epsilon_{0} \sqrt{t}\right\}}-I_{\left\{|y| \leq \epsilon_{0} \sqrt{t}\right\}}\right) \\
& +\bar{u}_{x}(x) e_{+}(t, y)+\mathbf{O}\left(t^{-1 / 2}[\ln (e+t)]^{2}\right) \mathbf{O}\left((1+|x|)^{-2}\right) \mathbf{O}(1+|y|) e^{-\frac{(x-y)^{2}}{M t}} \\
& +\mathbf{O}\left(t^{-1 / 2}(1+t)^{1 / 4}\right) \mathbf{O}\left((1+|x|)^{-1}\right) e^{-\frac{\left(\int_{K}^{x} \frac{d s}{a_{1}(s)}+\int_{K}^{y} \frac{d s}{a_{1}(s)}+t\right)^{2}}{M t}} I_{\left\{\left|\int_{K}^{y} \frac{d s}{a_{1}(s)}\right| \geq t\right\}} \\
& +\mathbf{O}\left(t^{-1 / 2}(1+t)^{1 / 4} \ln (e+t)\right) \mathbf{O}\left((1+|x|)^{-2}\right) e^{-\frac{\left(\int_{K}^{x} \frac{d s}{a_{1}(s)}+\int_{K}^{y} \frac{d s}{M} a_{1}^{(s)}+t\right)^{2}}{M t}} I_{\left\{\left|\int_{K}^{y} \frac{d s}{a_{1}(s)}\right| \geq t\right\}}, \\
& G_{y}(t, x ; y) \\
& =\mathbf{O}\left(t^{-1}\right) \mathbf{O}\left((1+|x|)^{-2}\right) \mathbf{O}(1+|y|) e^{-\frac{(x-y)^{2}}{M t}}+\bar{u}_{x}(x) \partial_{y} e_{+}(t, y), \\
& +\mathbf{O}\left(t^{-1}(1+t)^{-1 / 2} \ln (e+t)\right) \mathbf{O}\left((1+|x|)^{-1}\right) e^{-\frac{(x-y)^{2}}{M t}} \\
& +\mathbf{O}\left(t^{-1}(1+t)^{-1 / 2}\right) \mathbf{O}\left((1+|x|)^{-1}\right) \mathbf{O}(1+|y|) e^{-\frac{(x-y)^{2}}{M t}} \\
& +\mathbf{O}\left(t^{-1} \ln (e+t)\right) \mathbf{O}\left((1+|x|)^{-2}\right) e^{-\frac{(x-y)^{2}}{M t}}+\mathbf{O}\left(t^{-3 / 4} \ln (e+t)\right) \mathbf{O}\left(e^{-\eta|x-y|}\right) \\
& +\mathbf{O}\left(t^{-1}(1+t)^{1 / 4}\right) \mathbf{O}\left((1+|x|)^{-1}\right) e^{-\frac{\left(\int_{K}^{x} \frac{d s}{a_{1}(s)}+\int_{K}^{y} \frac{d s}{a_{1}(s)}+t\right)^{2}}{M t}} I_{\left\{\left|\int_{K}^{y} \frac{d s}{a_{1}(s)}\right| \geq t\right\}} ;
\end{aligned}
$$

(vi) $0<K \leq x \leq y$

$$
\begin{aligned}
G( & t, x ; y) \\
= & \mathbf{O}\left(t^{-1 / 2}\right) \mathbf{O}\left((1+|x|)^{-1}\right) \mathbf{O}(1+|y|) e^{-\frac{(x-y)^{2}}{M t}}+P_{+} \bar{u}_{x}\left(I_{\left\{|x-y| \leq \epsilon_{0} \sqrt{t}\right\}}-I_{\left\{|y| \leq \epsilon_{0} \sqrt{t}\right\}}\right) \\
& +\bar{u}_{x}(x) e_{+}(t, y)+\mathbf{O}\left(t^{-1 / 2}[\ln (e+t)]^{2}\right) \mathbf{O}\left((1+|x|)^{-1}\right) e^{-\frac{(x-y)^{2}}{M t}} \\
& +\mathbf{O}\left(t^{-1 / 2}\right) e^{\left.-\frac{\left(\int_{x}^{y} \frac{d s}{a_{1}(s)}+t\right.}{M t}\right)^{2}} \\
& +\mathbf{O}\left(\left(1+\left|\int_{x}^{y} \frac{d s}{a_{1}(s)}+t\right|+t^{1 / 2}\right)^{-3 / 2} \ln (e+t)\right) I_{\left\{\left|\int_{x}^{y} \frac{d s}{a_{1}(s)}\right| \leq t\right\}}
\end{aligned}
$$




$$
\begin{aligned}
& +\mathbf{O}\left(t^{-1 / 2}(1+t)^{1 / 4}\right) \mathbf{O}\left((1+|x|)^{-1}\right) e^{-\frac{\left(\int_{K}^{x} \frac{d s}{a_{1}(s)}+\int_{K}^{y} \frac{d s}{a_{1}(s)}+t\right)^{2}}{M t}} I_{\left\{\left|\int_{K}^{y} \frac{d s}{a_{1}(s)}\right| \geq t\right\}} \\
& +\mathbf{O}\left(t^{-1 / 2}(1+t)^{1 / 4} \ln (e+t)\right) \mathbf{O}\left((1+|x|)^{-2}\right) e^{-\frac{\left(\int_{K}^{x} \frac{d s}{a_{1}(s)}+\int_{K}^{y} \frac{d s}{a_{1}(s)}+t\right)^{2}}{M t}} I_{\left\{\left|\int_{K}^{y} \frac{d s}{a_{1}(s)}\right| \geq t\right\}} \\
& +\mathbf{O}\left(t^{-1 / 2}(1+t)^{1 / 4}\right) \mathbf{O}\left((1+|x|)^{-1}\right) e^{-\frac{\left(\int_{K}^{y} \frac{d s}{a_{1}(s)}+t\right)^{2}}{M t}} I_{\left\{\left|\int_{K}^{y} \frac{d s}{a_{1}(s)}\right| \leq t\right\}} \\
& +\mathbf{O}\left(t^{-1 / 2}(1+t)^{1 / 4} \ln (e+t)\right) \mathbf{O}\left((1+|x|)^{-2}\right) e^{-\frac{\left(\int_{K}^{y} \frac{d s}{a_{1}(s)}+t\right)^{2}}{M t}} I_{\left\{\left|\int_{K}^{y} \frac{d s}{a_{1}(s)}\right| \leq t\right\}} \\
& +\mathbf{O}\left(\left(1+\left|\int_{K}^{y} \frac{d s}{a_{1}(s)}+t\right|\right)^{-1 / 2}\right) \mathbf{O}\left((1+|x|)^{-1}\right) e^{-\frac{x^{2}}{M t}} I_{\left\{\left|\int_{K}^{y} \frac{d s}{a_{1}(s)}\right| \leq t\right\}} \\
& +\mathbf{O}\left(\left(1+\left|\int_{K}^{y} \frac{d s}{a_{1}(s)}+t\right|\right)^{-1 / 2} \ln (e+t)\right) \mathbf{O}\left((1+|x|)^{-2}\right) e^{-\frac{x^{2}}{M t}} I_{\left\{\left|\int_{K}^{y} \frac{d s}{a_{1}(s)}\right| \leq t\right\}}, \\
& G_{y}(t, x ; y) \\
& =\mathbf{O}\left(t^{-1}\right) \mathbf{O}\left((1+|x|)^{-1}\right) \mathbf{O}(1+|y|) e^{-\frac{(x-y)^{2}}{M t}}+\mathbf{O}\left(t^{-1}\right) e^{-\frac{\left(\int_{x}^{y} \frac{d s}{a_{1}(s)}+t\right)^{2}}{M t}} \\
& +\bar{u}_{x}(x) \partial_{y} e_{+}(t, y)+\mathbf{O}\left(\left(1+\left|\int_{x}^{y} \frac{d s}{a_{1}(s)}+t\right|+t^{1 / 2}\right)^{-5 / 2} \ln (e+t)\right) I_{\left\{\left|\int_{x}^{y} \frac{d s}{a_{1}(s)}\right| \leq t\right\}} \\
& +\mathbf{O}\left(t^{-1}(1+t)^{1 / 4}\right) \mathbf{O}\left((1+|x|)^{-1}\right) e^{-\frac{\left(\int_{K}^{x} \frac{d s}{a_{1}(s)}+\int_{K}^{y} \frac{d s}{a_{1}(s)}+t\right)^{2}}{M t}} I_{\left\{\left|\int_{K}^{y} \frac{d s}{a_{1}(s)}\right| \geq t\right\}} \\
& +\mathbf{O}\left(t^{-1}(1+t)^{1 / 4} \ln (e+t)\right) \mathbf{O}\left((1+|x|)^{-2}\right) e^{-\frac{\left(\int_{K}^{x} \frac{d s}{a_{1}(s)}+\int_{K}^{y} \frac{d s}{a_{1}(s)}+t\right)^{2}}{M{ }^{2}}} I_{\left\{\left|\int_{K}^{y} \frac{d s}{a_{1}(s)}\right| \geq t\right\}} \\
& +\mathbf{O}\left(t^{-1}(1+t)^{1 / 4}\right) \mathbf{O}\left((1+|x|)^{-1}\right) e^{-\frac{\left(\int_{K}^{y} \frac{d s}{a_{1}(s)}+t\right)^{2}}{M t}} I_{\left\{\left|\int_{K}^{y} \frac{d s}{a_{1}(s)}\right| \leq t\right\}} \\
& +\mathbf{O}\left(t^{-1}(1+t)^{1 / 4} \ln (e+t)\right) \mathbf{O}\left((1+|x|)^{-2}\right) e^{-\frac{\left(\int_{K}^{y} \frac{d s}{a_{1}(s)+t}\right)^{2}}{M t}} I_{\left\{\left|\int_{K}^{y} \frac{d s}{a_{1}(s)}\right| \leq t\right\}} \\
& +\mathbf{O}\left(\left(1+\left|\int_{K}^{y} \frac{d s}{a_{1}(s)}+t\right|\right)^{-3 / 2}\right) \mathbf{O}\left((1+|x|)^{-1}\right) e^{-\frac{x^{2}}{M t}} I_{\left\{\left|\int_{K}^{y} \frac{d s}{a_{1}(s)}\right| \leq t\right\}} \\
& +\mathbf{O}\left(\left(1+\left|\int_{K}^{y} \frac{d s}{a_{1}(s)}+t\right|\right)^{-3 / 2} \ln (e+t)\right) \mathbf{O}\left((1+|x|)^{-2}\right) e^{-\frac{x^{2}}{M t}} I_{\left\{\left|\int_{K}^{y} \frac{d s}{a_{1}(s)}\right| \leq t\right\}} .
\end{aligned}
$$

For $0 \leq y<K$, the integrals $\int_{K}^{y} \frac{d s}{a_{1}(s)}$ can be replaced by zero, and similarly for $x$.

We observe that the extensive number of summands in the cases $x, y \geq 0$ arises from the various interactions between the degenerate and nondegenerate characteristic speeds, and also from the two different decay rates $(1+|x|)^{-1}$ and $(1+|x|)^{-2} \ln (e+t)$. In practice, most of these terms give a negligible contribution in the nonlinear iteration of Howard (2005), but we record them here for completeness. 
Expressions of the form $\int_{K}^{x} \frac{d s}{a_{1}(s)}$ will be fully explained in the analysis; we point out here only that they are clearly necessary inasmuch as the behavior of $a_{1}(s)$ is only understood asymptotically, and the algebraic nature of the approach of $a_{1}(s)$ to $a_{1}^{+}$ precludes the possibilty of working (as in the nondegenerate case) with asymptotic endstates. We also mention that the behavior for small $t$ (that is, $|x-y| \geq \bar{K} t$ for some fixed constant $\bar{K}$ ) is of the form of heat kernels

$$
\mathbf{O}\left(t^{-1 / 2}\right) e^{-\frac{(x-y)^{2}}{M t}}
$$

which can be subsumed into the estimates of Theorem 1.1.

Of particular interest are the estimates on $G(t, x ; y)$ with decay rate $t^{-1 / 4}$. In the case $0<K \leq x \leq y$, the ODE Green's functions associated with degenerate decay in $x$ and nondegenerate decay in $y$ takes the form

$$
S_{\lambda}(x, y)=\mathbf{O}\left(\left|\lambda^{-1 / 2}\right|\right) \mathbf{O}\left(|x|^{-1}\right) e^{-\sqrt{\lambda} x-\int_{K}^{y} \mu_{3}(s ; \lambda) d s} .
$$

In the case $y=0$, we can proceed here as though analyzing the heat equation to immediately recover an estimate of the form

$$
\mathbf{O}\left(t^{-1 / 2}\right) \mathbf{O}\left(|x|^{-1}\right) e^{-\frac{x^{2}}{M t}} I_{\{y=0\}}
$$

The difficulty arises in the limit as $x$ goes to zero, in which case he have an estimate of the form

$$
S_{\lambda}(x, y)=\mathbf{O}\left(\left|\lambda^{-1 / 2}\right|\right) e^{-\int_{K}^{y} \mu_{3}(s ; \lambda) d s} .
$$

The exponent $\int_{K}^{y} \mu_{3}(s ; \lambda) d s$ is $\mathbf{O}(|\lambda|)$ and thus near $\lambda=0$ gives slower decay in $t$ than $e^{-\sqrt{\lambda} y}$. We observe, however, that this reduced $t$ decay occurs only for $|y| \geq a_{2}^{-} t$, and this allows the loss of decay in $t$ to be compensated by decay in $y$.

Regarding the excited terms, we note the relation

$$
e(t, y)=e_{-}(t, y) I_{\{y \leq 0\}}+e_{+}(t, y) I_{\{y>0\}} .
$$

Also, we observe that in the case $x \leq 0<K \leq y$, the term

$$
P_{+} \bar{u}_{x}(x) I_{\left\{|y| \leq \epsilon_{0} \sqrt{t}\right\}}
$$

arises naturally in the analysis, and is bounded by the expression

$$
\bar{u}_{x}(x) \mathbf{O}(1) e^{-\frac{y^{2}}{L t}} \text {. }
$$

In the cases $x, y \geq K>0$, the term that arises naturally in the analysis is

$$
P_{+} \bar{u}_{x}(x) I_{\left\{|x-y| \leq \epsilon_{0} \sqrt{t}\right\}},
$$

and we replace this with

$$
P_{+} \bar{u}_{x}(x) I_{\left\{|x-y| \leq \epsilon_{0} \sqrt{t}\right\}}=P_{+} \bar{u}_{x}(x) I_{\left\{|y| \leq \epsilon_{0} \sqrt{t}\right\}}+P_{+} \bar{u}_{x}(x)\left(I_{\left\{|x-y| \leq \epsilon_{0} \sqrt{t}\right\}}-I_{\left\{|y| \leq \epsilon_{0} \sqrt{t}\right\}}\right),
$$

where the first expression on the right-hand side of (1.9) is an expression of $e_{+}(y, t)$ and the second appears in $\widetilde{G}$. 


\section{ODE Estimates}

In this section we establish the critical estimates on $\varphi_{k}^{ \pm}(x ; \lambda)$, as well as on a choice of asymptotically growing solutions of (1.5) and dual solutions to both. Our eigenvalue ODE (1.5) takes the form

$$
\begin{aligned}
& v_{1_{x x}}-\left(a_{11}(x) v_{1}\right)_{x}-\left(a_{12}(x) v_{2}\right)_{x}=\lambda v_{1} \\
& v_{2_{x x}}-\left(a_{21}(x) v_{1}\right)_{x}-\left(a_{22}(x) v_{2}\right)_{x}=\lambda v_{2}
\end{aligned}
$$

where $a_{k j}(x)=\partial_{u_{j}} f_{k}\left(\bar{u}_{1}(x), \bar{u}_{2}(x)\right)$. Writing $V_{1}=v_{1}, V_{2}=v_{2}, V_{3}=v_{1_{x}}, V_{4}=v_{2_{x}}$, we have the first order system

$$
V^{\prime}=\mathbb{M}(x ; \lambda) V
$$

We will also be interested in the associated integrated equation

$$
\begin{aligned}
& w_{1_{x x}}-a_{11}(x) w_{1_{x}}-a_{12}(x) w_{2_{x}}=\lambda w_{1} \\
& w_{2_{x x}}-a_{21}(x) w_{1_{x}}-a_{22}(x) w_{2_{x}}=\lambda w_{2} .
\end{aligned}
$$

In particular, we will find it convenient when possible to compute growth and decay solutions of (2.1) by computing growth and decay solutions of (2.3) and computing their derivatives. We stress that from this point of view, no assumptions regarding integrability need be made. It is a question, rather, of scaling. we have

Writing (2.3) as a first order system with $W_{1}=w_{1}, W_{2}=w_{2}, W_{3}=w_{1_{x}}, W_{4}=w_{2_{x}}$,

$$
W^{\prime}=\mathbb{A}(x ; \lambda) W ; \quad ':=\partial_{x},
$$

where

$$
\mathbb{A}(x ; \lambda)=\left(\begin{array}{cccc}
0 & 0 & 1 & 0 \\
0 & 0 & 0 & 1 \\
\lambda & 0 & a_{11}(x) & a_{12}(x) \\
0 & \lambda & a_{21}(x) & a_{22}(x)
\end{array}\right),
$$

which has four eigenvalues $\mu_{k}(x ; \lambda)$ satisfying

$$
\begin{array}{ll}
\mu_{1}(x ; \lambda)=\frac{a_{1}(x)-\sqrt{a_{1}(x)^{2}+4 \lambda}}{2} ; & \mu_{2}(x ; \lambda)=\frac{a_{2}(x)-\sqrt{a_{2}(x)^{2}+4 \lambda}}{2}, \\
\mu_{3}(x ; \lambda)=\frac{a_{1}(x)+\sqrt{a_{1}(x)^{2}+4 \lambda}}{2} ; & \mu_{4}(x ; \lambda)=\frac{a_{2}(x)+\sqrt{a_{2}(x)^{2}+4 \lambda}}{2},
\end{array}
$$

with associated eigenvectors

$$
\begin{aligned}
P(x ; \lambda): & =\left(V_{1}, V_{2}, V_{3}, V_{4}\right) \\
& =\left(\begin{array}{cccc}
r_{1}(x) & r_{2}(x) & r_{1}(x) & r_{2}(x) \\
r_{1}(x) \mu_{1}(x, \lambda) & r_{2}(x) \mu_{2}(x ; \lambda) & r_{1}(x) \mu_{3}(x ; \lambda) & r_{2}(x) \mu_{4}(x ; \lambda)
\end{array}\right) .
\end{aligned}
$$


Here, $a_{1}(x) \leq a_{2}(x)$ are the eigenvalues of

$$
A(x)=\left(\begin{array}{ll}
a_{11}(x) & a_{12}(x) \\
a_{21}(x) & a_{22}(x)
\end{array}\right),
$$

namely

$$
\begin{aligned}
& a_{1}(x)=\frac{\operatorname{tr} A-\sqrt{(\operatorname{tr} A)^{2}-4 \operatorname{det} A}}{2}, \\
& a_{2}(x)=\frac{\operatorname{tr} A+\sqrt{(\operatorname{tr} A)^{2}-4 \operatorname{det} A}}{2},
\end{aligned}
$$

with associated eigenvectors $r_{k}=\left(1, \frac{a_{k}(x)-a_{11}(x)}{a_{12}(x)}\right)^{\operatorname{tr}}=\left(1, \frac{a_{k}(x) r_{k 2}(x)-a_{21}(x)}{a_{22}(x)}\right)^{\operatorname{tr}}$. At $x=+\infty$, we have $\mu_{1}^{+}(\lambda)=\mathbf{O}(1), \quad \mu_{2}^{+}(\lambda)=\mathbf{O}(\sqrt{\lambda}), \quad \mu_{3}(\lambda)=\mathbf{O}(\lambda)$, and $\mu_{4}^{+}(\lambda)=\mathbf{O}(\sqrt{\lambda})$, prompting our designation of $\mu_{1}$ and $\mu_{3}$ as nondegenerate modes, and $\mu_{2}$ and $\mu_{4}$ as degenerate modes. According to our assumption (H1) (and without loss of generality, taking $s=0$ and right side degeneracy), we have

$$
\operatorname{det} A_{+}=0, \quad \operatorname{tr} A_{+} \leq-\delta<0
$$

from which we observe that there exists a constant $K$ sufficiently large so that

$$
x>K \Rightarrow(\operatorname{tr} A)^{2}-4 \operatorname{det} A>0 .
$$

In the analysis that follows, we often proceed in the case $x \geq K$, observing that for $x \leq K$, all growth and decay solutions can be regarded as $\mathbf{O}(1)$ in $x$.

We note, prior to the statements of our lemmas, that the asymptotic limits $x \rightarrow \pm \infty$ of the $\mu_{k}(x ; \lambda)$ represent the growth and decay rates of solutions to both our unintegrated equation (2.1) and our integrated equation (2.3). Setting

$$
\mu_{k}^{ \pm}(\lambda)=\lim _{x \rightarrow \pm \infty} \mu_{k}(x ; \lambda)
$$

we have, for $|\lambda|$ sufficiently small,

$$
\begin{array}{ll}
\operatorname{Re} \mu_{1}^{-}(\lambda) \leq-\delta<0 & \operatorname{Re} \mu_{2}^{-}(\lambda)=\mathbf{O}(|\lambda|) \\
\operatorname{Re} \mu_{4}^{-}(\lambda) \geq+\delta>0 & \operatorname{Re} \mu_{3}^{-}(\lambda)=\mathbf{O}(|\lambda|) \\
\operatorname{Re} \mu_{1}^{+}(\lambda) \leq-\delta<0 & \operatorname{Re} \mu_{3}^{+}(\lambda)=\mathbf{O}(|\lambda|)
\end{array}
$$

Finally, we mention that throughout the analysis order, terms involving $|x|$ refer to behavior as $|x| \rightarrow \infty$, while order terms involving $\lambda$ refer to behavior as $|\lambda| \rightarrow 0$.

The following lemma is proven in Howard and Zumbrun (2004).

Lemma 2.1. Under the assumptions of Theorem 1.1, there exists some constant $r$ sufficiently small so that for $|\lambda| \leq r \lambda \neq \mathbb{R}_{-}$we have the following estimates on degenerate solutions of the integrated equation (2.3). 
For $x \geq 0, k=1,2$

$$
\begin{aligned}
W_{2 k}^{+}(x ; \lambda)= & e^{-\sqrt{\lambda} x}\left(\bar{u}_{k}(x)-u_{k}^{+}\right)\left(1+E_{2 k}(x ; \lambda)\right), \\
W_{2(k+2)}^{+}(x ; \lambda)= & e^{-\sqrt{\lambda} x}\left(\bar{u}_{k}(x)-u_{k}^{+}\right) \\
& \times\left(-\sqrt{\lambda}+\frac{\bar{u}_{k_{x}}(x)}{\bar{u}_{k}(x)-u_{k}^{+}}+E_{2(k+2)}(x ; \lambda)+\frac{\bar{u}_{k_{x}}(x)}{\bar{u}_{k}(x)-u_{k}^{+}} E_{2 k}(x ; \lambda)\right), \\
W_{4 k}^{+}(x ; \lambda)= & e^{+\sqrt{\lambda} x}\left(\bar{u}_{k}(x)-u_{k}^{+}\right)\left(1+E_{4 k}(x ; \lambda)\right), \\
W_{4(k+2)}^{+}(x ; \lambda)= & e^{+\sqrt{\lambda} x}\left(\bar{u}_{k}(x)-u_{k}^{+}\right) \\
& \times\left(+\sqrt{\lambda}+\frac{\bar{u}_{k_{x}}(x)}{\bar{u}_{k}(x)-u_{k}^{+}}+E_{4(k+2)}(x ; \lambda)+\frac{\bar{u}_{k_{x}}(x)}{\bar{u}_{k}(x)-u_{k}^{+}} E_{4(k-2)}(x ; \lambda)\right),
\end{aligned}
$$

where

$$
E_{2 k}(x ; \lambda), E_{4 k}(x ; \lambda)=\mathbf{O}\left(\left|\lambda^{1 / 2} \ln \lambda\right|\right) \wedge \mathbf{O}\left(|x|^{-1}\right), \quad k=1,2
$$

$(\wedge$ represents $\min )$, and

$$
E_{2 k}(x ; \lambda), E_{4 k}(x ; \lambda)=\mathbf{O}\left(\left|\lambda^{1 / 2}\right|\right) \mathbf{O}\left(|x|^{-1}\right), \quad k=3,4 .
$$

Additionally, we have for $\left|\lambda^{1 / 2} x\right| \leq 1$, the error estimates

$$
E_{j k}(x ; \lambda)=E_{j k}(0 ; \lambda)+\mathbf{O}\left(\left|\lambda^{1 / 2}\right|\right) \mathbf{O}(|x|) .
$$

Proof. The only statement in Lemma 2.1 not established in Howard and Zumbrun (2004) is the $\left|\lambda^{1 / 2} x\right| \leq 1$ estimate on $E_{j k}(x ; \lambda)$. Following the proof of Lemma 4.1 in Howard and Zumbrun (2004), we write $w_{1}(x)$ from Equation (2.3) as $w_{1}(x)=$ $\left(\bar{u}_{1}(x)-u_{1}^{+}\right) u_{1}(x)$, for which

$$
\begin{aligned}
u_{1}(x) & =e^{-\sqrt{\lambda} x}\left(1+E_{21}(x ; \lambda)\right), \\
u_{1_{x}}(x) & =e^{-\sqrt{\lambda} x}\left(-\sqrt{\lambda}+E_{23}(x ; \lambda)\right) .
\end{aligned}
$$

We have, then, the ODE for $E_{21}(x ; \lambda), \partial_{x} E_{21}(x ; \lambda)-\sqrt{\lambda} E_{21}(x ; \lambda)=E_{23}(x ; \lambda)$. Integrating, we find

$$
E_{21}(x ; \lambda)=e^{\sqrt{\lambda} x} E_{21}(0 ; \lambda)+\int_{0}^{x} e^{\sqrt{\lambda}(x-\xi)} E_{23}(\xi, \lambda) d \xi
$$

from which the estimate is clear. The remaining cases are similar.

We remark that the $\ln \lambda$ behavior in Lemma 2.1 appears in the analysis of single equations as well, and appears to be sharp (see Howard, 2002a; Howard, 2002b; Pego and Weinstein, 1992; Sandstede and Scheel, 2004).

Differentiating the estimates of Lemma 2.1, we obtain estimates on growth and decay solutions of the unintegrated equation (2.1).

Lemma 2.2. Under the assumptions of Theorem 1.1 and for $K$ large enough so that $x \geq K$ assures $(\operatorname{tr} A)^{2}-4 \operatorname{det} A \geq 0$, there exists some constant $r$ sufficiently small 
so that for $|\lambda| \leq r$, we have the following estimates on solutions of the unintegrated equation (2.2).

(i) $x \leq 0$ :

$$
\begin{aligned}
& \phi_{k}^{-}(x ; \lambda)=e^{\mu_{k+2}^{-}(\lambda) x}\left(V_{k+2}^{-}(\lambda)+\mathbf{O}\left(e^{-\alpha|x|}\right)\right), \\
& \psi_{k}^{-}(x ; \lambda)=e^{\mu_{k}^{-}(\lambda) x}\left(V_{k}^{-}(\lambda)+\mathbf{O}\left(e^{-\alpha|x|}\right)\right),
\end{aligned}
$$

where $k=1,2$, and $\mu_{k}^{-}(\lambda)$ and $V_{k}^{-}(\lambda)$ are eigenvalue-eigenvector pairs for the matrix $\mathbb{A}_{-}(\lambda):=\lim _{x \rightarrow-\infty} \mathbb{A}(x ; \lambda)$.

(ii) (nondegenerate solutions) For $x \geq K$ :

$$
\begin{aligned}
& \left(\phi_{11}^{+}, \phi_{12}^{+}\right)^{\operatorname{tr}}=: \Phi_{1}^{+}(x ; \lambda)=e^{\int_{K}^{x} \mu_{1}(s ; \lambda) d s}\left(r_{1}^{+}+\mathbf{O}\left(|x|^{-1}\right)\right), \\
\left(\phi_{11}^{+\prime}, \phi_{12}^{+\prime}\right)^{\operatorname{tr}}= & \left(\phi_{13}^{+}, \phi_{14}^{+}\right)^{\operatorname{tr}}=: \Phi_{1}^{+\prime}(x ; \lambda)=e^{\int_{K}^{x} \mu_{1}(s ; \lambda) d s}\left(r_{1}^{+} \mu_{1}^{+}(\lambda)+\mathbf{O}\left(|x|^{-1}\right)\right), \\
& \left(\psi_{11}^{+}, \psi_{12}^{+}\right)^{\operatorname{tr}}=: \Psi_{1}^{+}(x ; \lambda)=e^{\int_{K}^{x} \mu_{3}(s ; \lambda) d s}\left(r_{1}^{+}+\mathbf{O}\left(|x|^{-1}\right)\right), \\
\left(\psi_{11}^{+\prime}, \psi_{12}^{+\prime}\right)^{\operatorname{tr}}= & \left(\psi_{13}^{+}, \psi_{14}^{+}\right)^{\operatorname{tr}}=: \Psi_{1}^{+\prime}(x ; \lambda)=e^{\int_{K}^{x} \mu_{3}(s ; \lambda) d s}\left(r_{1}^{+} \mu_{3}^{+}(\lambda)+\mathbf{O}\left(|x|^{-1}\right)\right),
\end{aligned}
$$

while for $0 \leq x \leq K$ each term is $\mathbf{O}(1)$.

(iii) (degenerate solutions) For $x \geq 0, k=1,2$, and $\lambda \notin \mathbb{R}_{-}$:

$$
\begin{aligned}
\phi_{2 k}^{+}(x ; \lambda)= & e^{-\sqrt{\lambda} x}\left(\bar{u}_{k}(x)-u_{k}^{+}\right) \\
& \times\left(-\sqrt{\lambda}+\frac{\bar{u}_{k_{x}}(x)}{\bar{u}_{k}(x)-u_{k}^{+}}+E_{2(k+2)}(x ; \lambda)+\frac{\bar{u}_{k_{x}}(x)}{\bar{u}_{k}(x)-u_{k}^{+}} E_{2 k}(x ; \lambda)\right), \\
\phi_{2 k}^{+\prime}(x ; \lambda)= & e^{-\sqrt{\lambda} x}\left(\bar{u}_{k_{x x}}+\left(a_{k 1}(x) \bar{u}_{1_{x}} E_{21}+a_{k 2}(x) \bar{u}_{2_{x}} E_{22}\right)+\mathbf{O}\left(\left|\lambda^{1 / 2}\right|\right) \mathbf{O}\left(|x|^{-2}\right)\right. \\
& \left.+\mathbf{O}(|\lambda|) \mathbf{O}\left(|x|^{-1}\right)\right), \\
\psi_{2 k}^{+}(x ; \lambda)= & e^{+\sqrt{\lambda} x}\left(\bar{u}_{k}(x)-u_{k}^{+}\right) \\
& \times\left(+\sqrt{\lambda}+\frac{\bar{u}_{k_{x}}(x)}{\bar{u}_{k}(x)-u_{k}^{+}}+E_{4(k+2)}(x ; \lambda)+\frac{\bar{u}_{k_{x}}(x)}{\bar{u}_{k}(x)-u_{k}^{+}} E_{4 k}(x ; \lambda)\right), \\
\psi_{2 k}^{+\prime}(x ; \lambda)= & e^{+\sqrt{\lambda} x}\left(\bar{u}_{k_{x x}}+\left(a_{k 1}(x) \bar{u}_{1_{x}} E_{41}+a_{k 2}(x) \bar{u}_{2_{x}} E_{42}\right)\right. \\
& \left.+\mathbf{O}\left(\left|\lambda^{1 / 2}\right|\right) \mathbf{O}\left(|x|^{-2}\right)+\mathbf{O}(|\lambda|) \mathbf{O}\left(|x|^{-1}\right)\right),
\end{aligned}
$$

where the $E_{k j}(x ; \lambda)$ are as in Lemma 2.1 .

Proof. The estimates of Lemma 2.2 on degenerate solutions follow directly from Lemma 2.1 and Equation (2.3). For the nondegenerate solutions, standard theory suffices. (See, for example, Zumbrun and Howard, 1998/2002.)

Lemma 2.3. The Evans function associated with Equation (2.2), defined by

$$
D(\lambda):=\operatorname{det}\left(\phi_{1}^{-}(0 ; \lambda) \quad \phi_{2}^{-}(0 ; \lambda) \quad \phi_{1}^{+}(0 ; \lambda) \quad \phi_{2}^{+}(0 ; \lambda)\right)
$$


satisfies

$$
\begin{aligned}
D(\lambda) & =\operatorname{det}\left(\begin{array}{cccc}
\Phi_{1}^{+}(0 ; 0) & \Phi_{2}^{+}(0 ; 0) & \Phi_{1}^{-}(0 ; 0) & \Phi_{2}^{-}(0 ; 0) \\
\lambda \mathscr{W}_{1}^{+}(0 ; 0) & \lambda \mathscr{W}_{2}^{+}(0 ; 0) & -a_{1}^{-} r_{1}^{-} & \lambda \mathscr{W}_{2}^{-}(0 ; 0)
\end{array}\right)+\mathbf{O}\left(\left|\lambda^{3 / 2} \ln \lambda\right|\right) \\
& =: D_{a}(\lambda)+\mathbf{O}\left(\left|\lambda^{3 / 2} \ln \lambda\right|\right), \quad|\lambda| \rightarrow 0,
\end{aligned}
$$

where

$$
\Phi_{k}^{ \pm}=\left(\phi_{k 1}^{ \pm}, \phi_{k 2}^{ \pm}\right)^{\mathrm{tr}}
$$

Proof. Though Lemma 2.3 has been proven in Howard and Zumbrun (2004), we restate a brief account of the argument for later reference. This development follows that of Gardner and Zumbrun (1998).

Fast decay ODE solutions $\left(\Phi_{k}^{ \pm}(x ; 0)=\mathbf{O}\left(e^{-\alpha|x|}\right)\right)$. The fast decay solutions in this analysis are $\Phi_{2}^{-}$and $\Phi_{1}^{+}$. For the first, proceeding as in Gardner and Zumbrun (1998), we integrate

$$
\Phi_{2}^{-\prime \prime}-\left(A(x) \Phi_{2}^{-}\right)_{x}=\lambda \Phi_{2}^{-}
$$

on $(-\infty, x]$ to obtain

$$
\Phi_{2}^{-\prime}(x ; \lambda)-A(x) \Phi_{2}^{-}(x ; \lambda)=\lambda \int_{-\infty}^{x} \Phi(s ; \lambda)=: \lambda \mathscr{W}_{4}^{-}(x ; \lambda),
$$

where $\mathscr{W}_{2}^{-}(x ; \lambda)$ is analytic in $\lambda$. Similarly, for $\Phi_{1}^{+}$we have

$$
\Phi_{1}^{+\prime}(x ; \lambda)-A(x) \Phi_{1}^{+}(x ; \lambda)=\lambda \mathscr{W}_{1}^{+}(x ; \lambda),
$$

where $\mathscr{W}_{1}^{+}(x ; \lambda)$ is analytic in $\lambda$.

Slow decay ODE solutions $\left(\Phi_{1}^{+}(x ; 0)=\mathbf{O}(1)\right)$. The only slow decay solution in this analysis is $\Phi_{1}^{-}(x ; \lambda)$. Integrating again on $(-\infty, x]$, we have

$$
\Phi_{1}^{-1}(x ; \lambda)-A(x) \Phi_{1}^{-}(x ; \lambda)+A_{-} r_{1}^{-}=\lambda \mathscr{W}_{3}^{-}(x ; \lambda),
$$

where $\mathscr{W}_{3}^{-}(x ; \lambda)$ is analytic in $\lambda$, and $r_{1}^{-}$is the eigenvector of $A_{-}$associated with the eigenvalue $a_{1}^{-}$.

Degenerate decay ODE solutions $\left(\Phi_{2}^{+}(x ; 0)=\bar{u}_{x}(x)\right)$. Integrating our degenerate ODE solution on $[x,+\infty)$ we have by construction,

$$
\Phi_{2}^{+\prime}(x ; \lambda)-A(x) \Phi_{2}^{+}(x ; \lambda)=\lambda \mathscr{W}_{2}^{+}(x ; \lambda)=\lambda\left(W_{21}^{+}, W_{22}^{+}\right)^{\operatorname{tr}},
$$

where by Lemma $2.1 \mathscr{W}_{2}^{+}(0 ; \lambda)=\mathscr{W}_{2}^{+}(0,0)+\mathbf{O}\left(\left|\lambda^{1 / 2} \ln \lambda\right|\right)$.

Now, we compute

$$
\begin{aligned}
D(\lambda) & =\left.\operatorname{det}\left(\begin{array}{cccc}
\Phi_{1}^{+} & \Phi_{2}^{+} & \Phi_{1}^{-} & \Phi_{2}^{-} \\
\Phi_{1}^{+\prime} & \Phi_{2}^{+\prime} & \Phi_{1}^{-\prime} & \Phi_{2}^{-\prime}
\end{array}\right)\right|_{y=0} \\
& =\left.\operatorname{det}\left(\begin{array}{cccc}
\Phi_{1}^{+} & \Phi_{2}^{+} & \Phi_{1}^{-} & \Phi_{2}^{-} \\
A \Phi_{1}^{+}+\lambda \mathscr{W}_{1}^{+} & A \Phi_{2}^{+}+\lambda \mathscr{W}_{2}^{+} & A \Phi_{1}^{-}-a_{1}^{-} r_{1}^{-}+\lambda \mathscr{W}_{3}^{-} & A \Phi_{2}^{-}+\lambda \mathscr{W}_{4}^{-}
\end{array}\right)\right|_{y=0}
\end{aligned}
$$




$$
\begin{aligned}
& =\left.\operatorname{det}\left[\left(\begin{array}{cc}
I & 0 \\
A & I
\end{array}\right)\left(\begin{array}{cccc}
\Phi_{1}^{+} & \Phi_{2}^{+} & \Phi_{1}^{-} & \Phi_{2}^{-} \\
\lambda \mathscr{W}_{1}^{+} & \lambda \mathscr{W}_{2}^{+} & -a_{1}^{-} r_{1}^{-}+\lambda \mathscr{W}_{3}^{-} & \lambda \mathscr{W}_{2}^{-}
\end{array}\right)\right]\right|_{y=0} \\
& =\left.\operatorname{det}\left[\left(\begin{array}{cccc}
\Phi_{1}^{+} & \Phi_{2}^{+} & \Phi_{1}^{-} & \Phi_{2}^{-} \\
\lambda \mathscr{W}_{1}^{+} & \lambda \mathscr{W}_{2}^{+} & -a_{1}^{-} r_{1}^{-}+\lambda \mathscr{W}_{3}^{-} & \lambda \mathscr{W}_{2}^{-}
\end{array}\right)\right]\right|_{y=0} .
\end{aligned}
$$

From this final expression we see immediately that $D(0)=0$. The standard approach toward gaining higher order information on the Evans function at $\lambda=0$ involves differentiating this final expression with respect to $\lambda$ (see for example, Gardner and Zumbrun, 1998). Since in the case of a degenerate wave, the Evans function is not analytic at $\lambda=0$, we cannot follow this approach. As observed in Howard and Zumbrun (2004), however, the claim of Lemma 2.3 can be established directly from our detailed ODE estimates of Lemma 2.2.

In addition to the estimates of Lemma 2.2, we require estimates on the solutions dual to $\phi_{k}^{ \pm}$and $\psi_{k}^{ \pm}$. The ODE dual to (2.1) takes the form

$$
\begin{aligned}
& z_{1_{x x}}+a_{11}(x) z_{1_{x}}+a_{21}(x) z_{2_{x}}=\lambda z_{1} \\
& z_{2_{x x}}+a_{12}(x) z_{1_{x}}+a_{22}(x) z_{2_{x}}=\lambda z_{2},
\end{aligned}
$$

whose solutions we will denote $\tilde{\phi}_{k}^{ \pm}(x ; \lambda), \tilde{\psi}_{k}^{ \pm}(x ; \lambda)$. Denoting $Z_{1}=z_{1}, Z_{2}=z_{2}$, $Z_{3}=z_{1_{x}}$, and $Z_{4}=z_{2_{x}}$, we have the associated first order system

$$
Z^{\prime}=Z \tilde{\mathbb{A}}(x ; \lambda)
$$

where

$$
\tilde{\mathbb{A}}(x ; \lambda)=\left(\begin{array}{cccc}
0 & 0 & 1 & 0 \\
0 & 0 & 0 & 1 \\
\lambda & 0 & -a_{11}(x) & -a_{21}(x) \\
0 & \lambda & -a_{12}(x) & -a_{22}(x)
\end{array}\right) .
$$

We will employ the following lemma from Zumbrun and Howard (1998/2002).

Lemma 2.4. Suppose $V(x ; \lambda)$ satisfies $(2.2)$. Then $Z(x ; \lambda)$ is a solution of $(2.5)$ if and only if $Z S V=$ constant, where

$$
S(x)=\left(\begin{array}{cc}
-A(x) & I \\
-I & 0
\end{array}\right)
$$

According to Lemma 2.4, we can describe dual solutions through the relations

$$
\begin{array}{ll}
\tilde{\phi}_{j}^{+}(x ; \lambda) S(x) \psi_{k}^{+}(x ; \lambda)=\tilde{\psi}_{j}^{+}(x ; \lambda) S(x) \phi_{k}^{+}(x ; \lambda)=0, \quad \forall j, k=1,2 \\
\tilde{\phi}_{j}^{+}(x ; \lambda) S(x) \phi_{k}^{+}(x ; \lambda)=\tilde{\psi}_{j}^{+}(x ; \lambda) S(x) \psi_{k}^{+}(x ; \lambda)=0, \quad \forall j \neq k \\
\tilde{\phi}_{1}^{+}(x ; \lambda) S(x) \phi_{1}^{+}(x ; \lambda)=\tilde{\psi}_{1}^{+}(x ; \lambda) S(x) \psi_{1}^{+}(x ; \lambda)=1 \\
\tilde{\phi}_{2}^{+}(x ; \lambda) S(x) \phi_{2}^{+}(x ; \lambda)=\tilde{\psi}_{2}^{+}(x ; \lambda) S(x) \psi_{2}^{+}(x ; \lambda)=\lambda^{3 / 2},
\end{array}
$$


and similarly,

$$
\begin{aligned}
& \tilde{\phi}_{j}^{-}(x ; \lambda) S(x) \psi_{k}^{-}(x ; \lambda)=\tilde{\psi}_{j}^{-}(x ; \lambda) S(x) \phi_{k}^{-}(x ; \lambda)=0, \quad \forall j, k=1,2 \\
& \tilde{\phi}_{j}^{-}(x ; \lambda) S(x) \phi_{k}^{-}(x ; \lambda)=\tilde{\psi}_{j}^{-}(x ; \lambda) S(x) \psi_{k}^{-}(x ; \lambda)=\delta_{j k}, \quad \forall j, k=1,2 .
\end{aligned}
$$

We remark that the distinguished relations

$$
\tilde{\phi}_{2}^{+}(x ; \lambda) S(x) \phi_{2}^{+}(x ; \lambda)=\tilde{\psi}_{2}^{+}(x ; \lambda) S(x) \psi_{2}^{+}(x ; \lambda)=\lambda^{3 / 2},
$$

are taken as a result of the coalescence of $\phi_{2}^{+}(x ; \lambda)$ and $\psi_{2}^{+}(x ; \lambda)$ at $\lambda=0$ (see Lemma 2.2(iii)).

Accordingly, we have the following estimates on solutions of (2.4).

Lemma 2.5. Under the assumptions of Theorem 1.1, there exists some constant $r$ sufficiently small so that for $|\lambda| \leq r$, we have the following estimates on solutions of (2.4). (The order expression $\mathbf{O}_{a}(\cdot)$ refers to a term meromorphic in $|\lambda| \leq \delta_{s}$.)

(i) $x \leq 0$ :

$$
\begin{aligned}
& \tilde{\phi}_{k}^{-}(x ; \lambda)=e^{-\mu_{k+2}^{-}(\lambda) x}\left(\widetilde{V}_{k+2}^{-}(\lambda)+\mathbf{O}\left(e^{-\alpha|x|}\right)\right), \\
& \tilde{\psi}_{k}^{-}(x ; \lambda)=e^{-\mu_{k}^{-}(\lambda) x}\left(\widetilde{V}_{k}^{-}(\lambda)+\mathbf{O}\left(e^{-\alpha|x|}\right)\right),
\end{aligned}
$$

where $k=1,2$, the $\mu_{k}^{-}(\lambda)$ are as in Lemma 2.2 , and $\widetilde{V}_{j}^{-}(\lambda)$ are the eigenvectors of $\lim _{x \rightarrow-\infty} \tilde{\mathbb{A}}(x ; \lambda)$, scaled so that $\widetilde{V}_{j}^{ \pm}(\lambda) V_{k}^{ \pm}(\lambda)=\delta_{k}^{j}$. Moreover, $\tilde{\psi}_{23}^{-}(x ; \lambda), \tilde{\psi}_{24}^{-}(x ; \lambda)$, $\tilde{\phi}_{13}^{-}(x ; \lambda)$, and $\tilde{\phi}_{14}^{-}(x ; \lambda)$ are all $\mathbf{O}_{a}(|\lambda|)$.

(ii) (nondegenerate solutions) For $x \geq K, k=1,2$ :

$$
\begin{aligned}
\tilde{\phi}_{1 k}^{+}(x ; \lambda) & =e^{-\int_{K}^{x} \mu_{1}(s ; \lambda) d s}\left[\mathbf{O}_{a}(1)+\mathbf{O}\left(\left|\lambda^{1 / 2} \ln \lambda\right|\right)\right] \\
\tilde{\phi}_{1 k}^{+\prime}(x ; \lambda)=\tilde{\phi}_{1(k+2)}^{+}(x ; \lambda) & =e^{-\int_{K}^{x} \mu_{1}(s ; \lambda) d s}\left[\mathbf{O}_{a}(1)+\mathbf{O}\left(\left|\lambda^{1 / 2} \ln \lambda\right|\right)\right] . \\
\tilde{\psi}_{1 k}^{+}(x ; \lambda) & =e^{-\int_{K}^{x} \mu_{3}(s ; \lambda) d s}\left[\mathbf{O}_{a}(1)+\mathbf{O}\left(\left|\lambda^{1 / 2} \ln \lambda\right|\right)\right] \\
\tilde{\psi}_{1 k}^{+\prime}=\tilde{\psi}_{1(k+2)}^{+}(x ; \lambda) & =e^{-\int_{K}^{x} \mu_{3}(s ; \lambda) d s}\left[\mathbf{O}_{a}(|\lambda|)+\mathbf{O}\left(\left|\lambda^{3 / 2} \ln \lambda\right|\right)\right],
\end{aligned}
$$

while for $x \leq K$ each expression is $\mathbf{O}(1)$, except $\tilde{\psi}_{1 k}^{+\prime}$, which is $\mathbf{O}(|\lambda|)$. (iii) degenerate solutions $x \geq 0$ :

$$
\begin{aligned}
& \tilde{\phi}_{2 k}^{+}(x ; \lambda)=e^{\sqrt{\lambda} x}\left(\mathbf{O}_{a}(1)+\mathbf{O}\left(\left|\lambda^{1 / 2}\right|\right) \mathbf{O}(|x|)+\mathbf{O}\left(\left|\lambda^{1 / 2} \ln \lambda\right|\right)\right) \\
& \tilde{\phi}_{2 k}^{+\prime}(x ; \lambda)=\tilde{\phi}_{2(k+1)}^{+}(x ; \lambda)=e^{\sqrt{\lambda} x} \mathbf{O}(|\lambda|) \mathbf{O}(|x|) \\
& \tilde{\psi}_{2 k}^{+}(x ; \lambda)=e^{-\sqrt{\lambda} x}\left(\mathbf{O}_{a}(1)+\mathbf{O}\left(\left|\lambda^{1 / 2}\right|\right) \mathbf{O}(|x|)+\mathbf{O}\left(\left|\lambda^{1 / 2} \ln \lambda\right|\right)\right) \\
& \tilde{\psi}_{2 k}^{+\prime}(x ; \lambda)=\tilde{\psi}_{2(k+2)}^{+}(x ; \lambda)=e^{-\sqrt{\lambda} x} \mathbf{O}(|\lambda|) \mathbf{O}(|x|),
\end{aligned}
$$

where $k=1,2$.

Proof. The case $x \leq 0$ is nondegenerate, and since the solutions do not coalesce at $\lambda=0$, straightforward (see, for example, Zumbrun and Howard, 1998/2002). The only new assertion regards the $\mathbf{O}(|\lambda|)$ behavior of $\widetilde{\Psi}_{2}^{-\prime}(y ; \lambda)$ and $\widetilde{\Phi}_{1}^{-\prime}(y ; \lambda)$. Such 
behavior is most directly observed by choosing the fast growth solution $\psi_{1}^{-}(x ; \lambda)$ as the derivative of a solution to the integrated equation (2.3). In this way, we insure by construction that

$$
\Psi_{1}^{-\prime}-A \Psi_{1}^{-}=\mathbf{O}(|\lambda|) e^{\mu_{1}^{-}(\lambda) x}
$$

Then, according to (2.7), we must have

$$
\widetilde{M}^{-}(x ; \lambda) \tilde{\psi}_{2}^{-}(x ; \lambda)^{\operatorname{tr}}=(0,0,0,1)^{\operatorname{tr}},
$$

where, omitting independent variables for brevity,

$$
\widetilde{M}^{ \pm}(x ; \lambda)=\left(\begin{array}{llll}
-a_{11} \phi_{11}^{ \pm}-a_{12} \phi_{12}^{ \pm}+\phi_{13}^{ \pm} & -a_{21} \phi_{11}^{ \pm}-a_{22} \phi_{12}^{ \pm}+\phi_{14}^{ \pm} & -\phi_{11}^{ \pm} & -\phi_{12}^{ \pm} \\
-a_{11} \phi_{21}^{ \pm}-a_{12} \phi_{22}^{ \pm}+\phi_{23}^{ \pm} & -a_{21} \phi_{21}^{ \pm}-a_{22} \phi_{22}^{ \pm}+\phi_{24}^{ \pm} & -\phi_{21}^{ \pm} & -\phi_{22}^{ \pm} \\
-a_{11} \psi_{11}^{ \pm}-a_{12} \psi_{12}^{ \pm}+\psi_{13}^{ \pm} & -a_{21} \psi_{11}^{ \pm}-a_{22} \psi_{12}^{ \pm}+\psi_{14}^{ \pm} & -\psi_{11}^{ \pm} & -\psi_{12}^{ \pm} \\
-a_{11} \psi_{21}^{ \pm}-a_{12} \psi_{22}^{ \pm}+\psi_{23}^{ \pm} & -a_{21} \psi_{21}^{ \pm}-a_{22} \psi_{22}^{ \pm}+\psi_{24}^{ \pm} & -\psi_{21}^{ \pm} & -\psi_{22}^{ \pm}
\end{array}\right) .
$$

Applying Cramer's rule and transposing the matrices, we find

$$
\begin{aligned}
& \tilde{\psi}_{23}^{-}(x ; \lambda)
\end{aligned}
$$

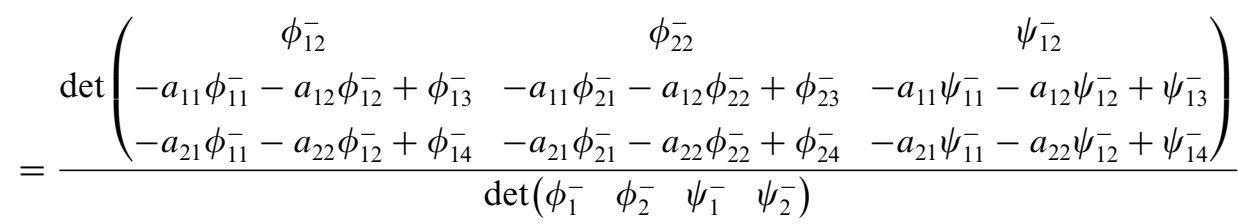

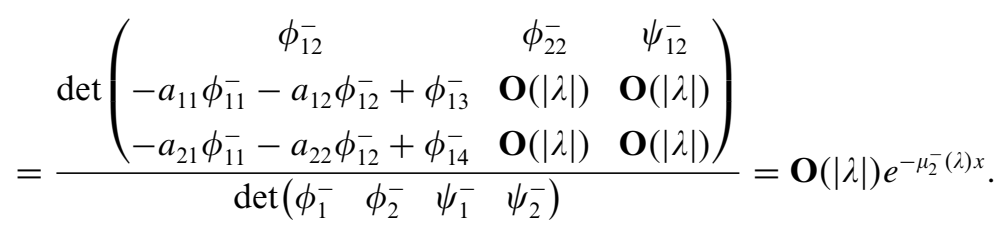

The calculations for $\tilde{\psi}_{24}^{-}(x ; \lambda), \tilde{\phi}_{13}^{-}(x ; \lambda)$, and $\tilde{\phi}_{14}^{-}(x ; \lambda)$ are similar.

For $x \geq 0$, the analysis is considerably more delicate, and we carry out a full analysis for the cases $\tilde{\phi}_{1}^{+}(x ; \lambda)$ and $\tilde{\phi}_{2}^{+}(x ; \lambda)$ only. For $\tilde{\phi}_{1}^{+}(x ; \lambda)$, from $(2.6)$ we have the relation

$$
\widetilde{M}(x ; \lambda) \tilde{\phi}_{1}^{+}(x ; \lambda)^{\operatorname{tr}}=(1,0,0,0)^{\operatorname{tr}} .
$$

Applying Cramer's rule, we find

$$
\begin{aligned}
& \tilde{\phi}_{13}^{+}(x ; \lambda) \\
& =\frac{\operatorname{det}\left(\begin{array}{ccc}
\phi_{22}^{+} & \psi_{12}^{+} & \psi_{21}^{+} \\
-a_{11} \phi_{21}^{+}-a_{12} \phi_{22}^{+}+\phi_{23}^{+} & -a_{11} \psi_{11}^{+}-a_{12} \psi_{12}^{+}+\psi_{13}^{+} & -a_{11} \psi_{21}^{+}-a_{12} \psi_{22}^{+}+\psi_{23}^{+} \\
-a_{21} \phi_{21}^{+}-a_{22} \phi_{22}^{+}+\phi_{24}^{+} & -a_{21} \psi_{11}^{+}-a_{22} \psi_{12}^{+}+\psi_{14}^{+} & -a_{21} \psi_{21}^{+}-a_{22} \psi_{22}^{+}+\psi_{24}^{+}
\end{array}\right)}{\operatorname{det}\left(\phi_{1}^{+} \phi_{2}^{+} \psi_{1}^{+} \psi_{2}^{+}\right)}
\end{aligned}
$$


We observe now that by our construction from the integrated equation (2.3), we have the reduced form

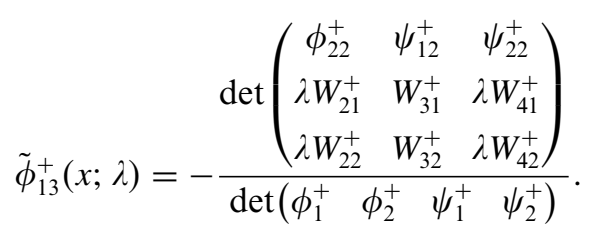

Computing directly, we observe that the numerator has the form

$$
\lambda W_{31}^{+}\left(\phi_{22}^{+} W_{42}^{+}-\psi_{22}^{+} W_{22}^{+}\right)+\lambda^{2} \psi_{12}^{+}\left(W_{41}^{+} W_{22}^{+}-W_{21}^{+} W_{42}^{+}\right)+\lambda W_{32}^{+}\left(\psi_{22}^{+} W_{21}^{+}-\phi_{22}^{+} W_{41}^{+}\right) .
$$

For the terms in parentheses, we have cancellation, exemplified by the calculation,

$$
\begin{aligned}
\phi_{22}^{+} W_{42}^{+}-\psi_{22}^{+} W_{22}^{+}=\left(\bar{u}_{2}(x)-u_{2}^{+}\right)^{2}\left[\left(-\sqrt{\lambda}+\frac{\bar{u}_{2_{x}}}{\bar{u}_{2}-u_{2}^{+}}+E_{24}+\frac{\bar{u}_{2_{x}}}{\bar{u}_{2}-u_{2}^{+}} E_{22}\right)\left(1+E_{42}\right)\right. \\
\left.-\left(\sqrt{\lambda}+\frac{\bar{u}_{2_{x}}}{\bar{u}_{2}-u_{2}^{+}}+E_{44}+\frac{\bar{u}_{2_{x}}}{\bar{u}_{2}-u_{2}^{+}} E_{42}\right)\left(1+E_{22}\right)\right] \\
=\left(\bar{u}_{2}(x)-u_{2}^{+}\right)^{2}\left[-2 \sqrt{\lambda}+\left(E_{24}-E_{44}\right)-\sqrt{\lambda}\left(E_{42}+E_{22}\right)\right. \\
\left.+\left(E_{42} E_{24}-E_{22} E_{44}\right)\right] \\
=\mathbf{O}\left(|x|^{-2}\right) \mathbf{O}\left(\left|\lambda^{1 / 2}\right|\right)+\mathbf{O}\left(|x|^{-2}\right) \mathbf{O}(|\lambda \ln \lambda|),
\end{aligned}
$$

with similar estimates on the remaining terms. We conclude that the numerator has the form

$$
e^{\int_{K}^{x} \mu_{3}(s ; \lambda) d s}\left[\mathbf{O}\left(|x|^{-2}\right) \mathbf{O}\left(\left|\lambda^{3 / 2}\right|\right)+\mathbf{O}\left(|x|^{-2}\right) \mathbf{O}\left(\left|\lambda^{2} \ln \lambda\right|\right)\right]
$$

Computing directly (i.e. expanding the determinants), according to the estimates of Lemmas 2.1 and 2.2, we find

$$
\begin{aligned}
& \operatorname{det}\left(\begin{array}{llll}
\phi_{1}^{+} & \phi_{2}^{+} & \psi_{1}^{+} & \psi_{2}^{+}
\end{array}\right) \\
& =e^{\int_{K}^{x} \mu_{1}(s ; \lambda)+\mu_{3}(s ; \lambda) d s}\left(\operatorname{ord}\left(\lambda^{3 / 2}\right) \operatorname{ord}\left(|x|^{-2}\right)+\operatorname{ord}(\lambda) \operatorname{ord}\left(|x|^{-3}\right) \widetilde{E}(x ; \lambda)\right),
\end{aligned}
$$

where by ord( ) we mean strict order, bounded above and below (hence a term we can divide by), and by $\widetilde{E}$ we mean

$$
\widetilde{E}(x ; \lambda)=E_{41}(x ; \lambda)+E_{22}(x ; \lambda)-E_{42}(x ; \lambda)-E_{21}(x ; \lambda)=\mathbf{O}\left(\left|\lambda^{1 / 2} \ln \lambda\right|\right) \wedge \mathbf{O}\left(|x|^{-1}\right) .
$$

Combining these representations, we find that

$$
\tilde{\phi}_{13}^{+}(x ; \lambda)=e^{-\int_{K}^{x} \mu_{1}(s ; \lambda) d s}\left[\mathbf{O}_{a}(1)+\mathbf{O}\left(\left|\lambda^{1 / 2} \ln \lambda\right|\right)\right] .
$$

Integrating $\tilde{\phi}_{13}^{+}(x ; \lambda)$ on $[x,+\infty)$, we obtain a similar estimate on $\tilde{\phi}_{11}^{+}(x ; \lambda)$. For $\tilde{\phi}_{2}^{+}(x ; \lambda)$, we have

$$
\widetilde{M}(x ; \lambda) \tilde{\phi}_{2}^{+}(x ; \lambda)^{\operatorname{tr}}=\left(0, \lambda^{3 / 2}, 0,0\right)^{\operatorname{tr}}
$$


Applying Cramer's rule, we find

$$
\begin{gathered}
\tilde{\phi}_{21}^{+}(x ; \lambda) \\
\operatorname{det}\left(\begin{array}{ccc}
\phi_{11}^{+} & \psi_{11}^{+} & \psi_{21}^{+} \\
\phi_{12}^{+} & \psi_{12}^{+} & \psi_{22}^{+} \\
-a_{21} \phi_{11}^{+}-a_{22} \phi_{12}^{+}+\phi_{14}^{+} & -a_{21} \psi_{11}^{+}-a_{22} \psi_{12}^{+}+\psi_{14}^{+} & -a_{21} \psi_{21}^{+}-a_{22} \psi_{22}^{+}+\psi_{24}^{+}
\end{array}\right) \\
\operatorname{det}\left(\phi_{1}^{+} \phi_{2}^{+} \psi_{1}^{+} \psi_{2}^{+}\right)
\end{gathered}
$$

We observe now that by our construction from the integrated equation (2.3), we have the reduced form

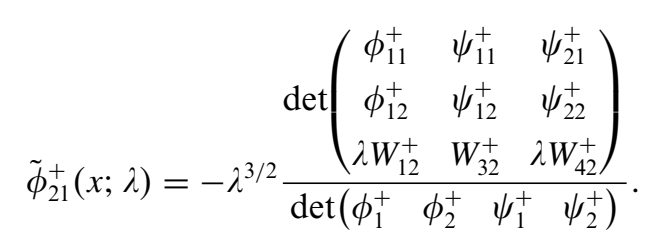

Expanding as in the case $\tilde{\phi}_{21}^{+}(x ; \lambda)$, we have

$$
\begin{aligned}
& \operatorname{det}\left(\begin{array}{ccc}
\phi_{11}^{+} & \psi_{11}^{+} & \psi_{21}^{+} \\
\phi_{12}^{+} & \psi_{12}^{+} & \psi_{22}^{+} \\
\lambda W_{12}^{+} & W_{32}^{+} & \lambda W_{42}^{+}
\end{array}\right) \\
& =e^{\sqrt{\lambda} x+\int_{K}^{x}\left(\mu_{1}(s ; \lambda)+\mu_{3}(x ; \lambda)\right) d s}\left(\mathbf{O}\left(|x|^{-2}\right)+\mathbf{O}\left(|\lambda|^{1 / 2}\right) \mathbf{O}\left(|x|^{-1}\right)+\mathbf{O}\left(\left|\lambda^{1 / 2} \ln \lambda\right|\right)\right),
\end{aligned}
$$

from which our estimate on $\tilde{\phi}_{21}^{+}(x ; \lambda)$ is immediate. The remaining cases are similar.

Lemma 2.6. Under the assumptions of Lemma 2.5, we observe the following critical cancellation estimates, for which, as in Lemma 2.5, the expression $\mathbf{O}_{a}(\cdot)$ refers to terms meromorphic in a neighborhood of $\lambda=0$.

(i) For $k=1,2$,

$$
\lambda^{-3 / 2}\left(E_{4 k}(0 ; \lambda)-E_{2 k}(0 ; \lambda)\right)+m(\lambda)=\mathbf{O}_{a}\left(\left|\lambda^{-1}\right|\right)+\mathbf{O}\left(\left|\lambda^{-1 / 2}(\ln \lambda)^{2}\right|\right) ;
$$

(ii) $d_{22}^{+}(\lambda) \Phi_{2}^{+}(x ; \lambda)-\lambda^{-3 / 2} \Psi_{2}^{+}(x ; \lambda)$

$$
=e^{\sqrt{\lambda} x}\left(\frac{P_{+} \bar{u}_{x}}{\lambda}+\mathbf{O}\left(\left|\lambda^{-1 / 2}(\ln \lambda)^{2}\right|\right) \mathbf{O}\left(|x|^{-1}\right)+\mathbf{O}\left(\left|\lambda^{-1 / 2}\right|\right) \wedge \mathbf{O}\left(\left|\lambda^{-1}\right|\right) \mathbf{O}\left(|x|^{-1}\right)\right)
$$

(iii) $d_{22}^{+}(\lambda) \widetilde{\Psi}_{2}^{+}(x ; \lambda)+\lambda^{-3 / 2} \widetilde{\Phi}_{2}^{+}(x ; \lambda)$

$$
\begin{gathered}
=e^{\sqrt{\lambda} x}\left(\mathbf{O}_{a}\left(\left|\lambda^{-1}\right|\right)+\mathbf{O}\left(\left|\lambda^{-1 / 2}(\ln \lambda)^{2}\right|\right) \mathbf{O}(|x|)+\left(\mathbf{O}\left(\left|\lambda^{-1 / 2}\right|\right) \mathbf{O}\left(|x|^{2}\right)\right)\right. \\
\left.\wedge\left(\mathbf{O}\left(\left|\lambda^{-1}\right|\right) \mathbf{O}\left(|x|^{-1}\right)\right)\right)
\end{gathered}
$$

(iv) $d_{22}^{+}(\lambda) \widetilde{\Psi}_{2}^{+\prime}(x ; \lambda)+\lambda^{-3 / 2} \widetilde{\Phi}_{2}^{+\prime}(x ; \lambda)$

$$
=e^{\sqrt{\lambda} x}\left(\mathbf{O}(|\ln \lambda|)+\mathbf{O}\left(\left|\lambda^{1 / 2}(\ln \lambda)^{2}\right|\right) \mathbf{O}(|x|)+\left(\mathbf{O}\left(\left|\lambda^{1 / 2}\right|\right) \mathbf{O}\left(|x|^{2}\right)\right) \wedge(\mathbf{O}(|x|))\right),
$$


where $m(\lambda)$ and $d_{22}^{+}(\lambda)$ are defined through the relation

$$
d_{22}^{+}(\lambda)=\lambda^{-3 / 2} \frac{\operatorname{det}\left(\begin{array}{llll}
\phi_{1}^{+} & \psi_{2}^{+} & \phi_{1}^{-} & \phi_{2}^{-}
\end{array}\right)}{\operatorname{det}\left(\begin{array}{llll}
\phi_{1}^{+} & \phi_{2}^{+} & \phi_{1}^{-} & \phi_{2}^{-}
\end{array}\right)}=\lambda^{-3 / 2}+m(\lambda),
$$

for which $m(\lambda)=\mathbf{O}\left(\left|\lambda^{-1} \ln \lambda\right|\right)$.

Proof. The cancellation in Lemma 2.6 arises from the coalescing of the decay and growth degenerate solutions. In the case of nondegenerate waves, independence of these solutions plays a critical role in the determination of the behavior of the scattering coefficients (see, for example, Zumbrun and Howard, 1998/2002). Here, rather, the scattering coefficients must be understood first so that the extent of the dependence can be observed.

We begin by observing that the estimate (2.8) on the scattering coefficient $d_{22}^{+}(\lambda)$ (and on $m(\lambda)$ ) is a direct result of the relationship

$$
\phi_{2}^{+}(0 ; \lambda)=\psi_{2}^{+}(0 ; \lambda)+\mathbf{O}\left(\left|\lambda^{1 / 2} \ln \lambda\right|\right)
$$

which is immediate from the estimates of Lemma 2.2. In particular, since $d_{22}^{+}(\lambda)$ does not depend on $x$, we can evaluate the determinant quotient at any value of $x$, and we evaluate it at $x=0$.

Taking $k=1$ for definiteness in (i), we first compute

$$
\begin{aligned}
d_{22}^{+} & (\lambda) \Phi_{21}^{+}(x ; \lambda)-\lambda^{-3 / 2} \Psi_{21}^{+}(x ; \lambda) \\
= & \left(\lambda^{-3 / 2}+m(\lambda)\right) \Phi_{21}^{+}(x ; \lambda)-\lambda^{-3 / 2} \Psi_{21}^{+}(x ; \lambda) \\
= & \left(\lambda^{-3 / 2}+m(\lambda)\right) e^{-\sqrt{\lambda} x}\left(\bar{u}_{1}(x)-u_{1}^{+}\right) \\
& \times\left(-\sqrt{\lambda}+\frac{\bar{u}_{1_{x}}}{\bar{u}_{1}-u_{1}^{+}}+E_{23}(x ; \lambda)+\frac{\bar{u}_{1_{x}}}{\bar{u}_{1}-u_{1}^{+}} E_{21}(x ; \lambda)\right) \\
& -\lambda^{-3 / 2} e^{\sqrt{\lambda} x}\left(\bar{u}_{1}(x)-u_{1}^{+}\right)\left(\sqrt{\lambda}+\frac{\bar{u}_{1_{x}}}{\bar{u}_{1}-u_{1}^{+}}+E_{43}(x ; \lambda)+\frac{\bar{u}_{1_{x}}}{\bar{u}_{1}-u_{1}^{+}} E_{41}(x ; \lambda)\right) .
\end{aligned}
$$

In the case $|\sqrt{\lambda} x| \leq 1$, we have

$$
\begin{aligned}
\left(\lambda^{-3 / 2}\right. & +m(\lambda))(1+\mathbf{O}(|\sqrt{\lambda} x|))\left(\bar{u}_{1}(x)-u_{1}^{+}\right) \\
& \times\left(-\sqrt{\lambda}+\frac{\bar{u}_{1_{x}}}{\bar{u}_{1}-u_{1}^{+}}+E_{23}(x ; \lambda)+\frac{\bar{u}_{1_{x}}}{\bar{u}_{1}-u_{1}^{+}} E_{21}(x ; \lambda)\right) \\
& -\lambda^{-3 / 2}(1+\mathbf{O}(|\sqrt{\lambda} x|))\left(\bar{u}_{1}(x)-u_{1}^{+}\right) \\
& \times\left(\sqrt{\lambda}+\frac{\bar{u}_{1_{x}}}{\bar{u}_{1}-u_{1}^{+}}+E_{43}(x ; \lambda)+\frac{\bar{u}_{1_{x}}}{\bar{u}_{1}-u_{1}^{+}} E_{41}(x ; \lambda)\right) \\
= & \mathbf{O}_{a}\left(\left|\lambda^{-1}\right|\right) \mathbf{O}\left(|x|^{-1}\right)+\bar{u}_{1_{x}}(x)\left(\left(E_{21}(x ; \lambda)-E_{41}(x ; \lambda)\right) \lambda^{-3 / 2}+m(\lambda)\right) \\
& +\mathbf{O}\left(\left|\lambda^{-1 / 2}(\ln \lambda)^{2}\right|\right) \mathbf{O}\left(|x|^{-1}\right)+\mathbf{O}\left(\left|\lambda^{-1 / 2}\right|\right) \wedge \mathbf{O}\left(\left|\lambda^{-1}\right|\right) \mathbf{O}\left(|x|^{-1}\right) .
\end{aligned}
$$

According to Lemma 2.3, spectral stability implies

$$
d_{22}^{+}(\lambda) \Phi_{21}^{+}(0 ; \lambda)-\lambda^{-3 / 2} \Psi_{21}^{+}(0 ; \lambda)=\mathbf{O}_{a}\left(\left|\lambda^{-1}\right|\right)+\mathbf{O}\left(\left|\lambda^{-1 / 2} \ln \lambda\right|\right) .
$$


Combining these last two equalities, we conclude (i). Recalling from Lemma 2.1 the relations $E_{j k}(x ; \lambda)=E_{j k}(0 ; \lambda)+\mathbf{O}\left(\left|\lambda^{1 / 2}\right|\right) \mathbf{O}(|x|)$, we have

$$
\begin{aligned}
& \bar{u}_{1_{x}}(x)\left(\left(E_{21}(x ; \lambda)-E_{41}(x ; \lambda)\right) \lambda^{-3 / 2}+m(\lambda)\right) \\
& =\bar{u}_{1_{x}}(x)\left(\left(E_{21}(0 ; \lambda)-E_{41}(0 ; \lambda)\right) \lambda^{-3 / 2}+m(\lambda)+\mathbf{O}\left(\left|\lambda^{1 / 2}\right|\right) \mathbf{O}(|x|) \lambda^{-3 / 2}\right) \\
& =\bar{u}_{1_{x}}(x)\left(\mathbf{O}_{a}\left(\left|\lambda^{-1}\right|\right)+\mathbf{O}\left(\left|\lambda^{-1 / 2} \ln \lambda\right|\right)+\mathbf{O}_{a}\left(\left|\lambda^{-1}\right|\right) \mathbf{O}(|x|)\right),
\end{aligned}
$$

from which we conclude (ii). In the case $|\sqrt{\lambda} x| \geq 1$, we observe that $|x|^{-1} \leq \sqrt{\lambda}$ from which (ii) follows immediately from (2.9).

For estimate (iii), we observe as in the proof of Lemma 2.5 that

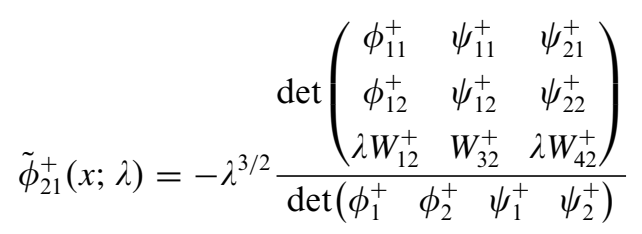

$$
\begin{aligned}
& =\frac{-\lambda^{3 / 2}}{\operatorname{det}\left(\phi_{1}^{+} \quad \phi_{2}^{+} \quad \psi_{1}^{+} \quad \psi_{2}^{+}\right)}\left[W_{32}^{+}\left(\psi_{21}^{+} \phi_{12}^{+}-\phi_{11}^{+} \psi_{22}^{+}\right)+\mathbf{O}(|\lambda|)\right]
\end{aligned}
$$

with also

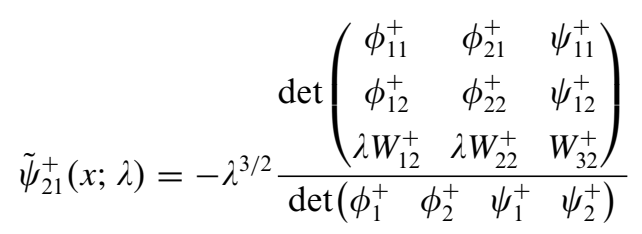

$$
\begin{aligned}
& =\frac{-\lambda^{3 / 2}}{\operatorname{det}\left(\phi_{1}^{+} \quad \phi_{2}^{+} \quad \psi_{1}^{+} \quad \psi_{2}^{+}\right)}\left[W_{32}^{+}\left(\phi_{11}^{+} \phi_{22}^{+}-\phi_{21}^{+} \phi_{12}^{+}\right)+\mathbf{O}(|\lambda|)\right] \text {. }
\end{aligned}
$$

We have, then,

$$
\begin{aligned}
d_{22}^{+}(\lambda)+\tilde{\psi}_{2}^{+}(x ; \lambda)+\lambda^{-3 / 2} \tilde{\phi}_{2}^{+}(x ; \lambda) \\
=-\frac{\lambda^{3 / 2}\left(\psi_{11}^{+}-a_{11}(x) \psi_{11}^{+}-a_{12}(x) \psi_{12}^{+}\right)}{\operatorname{det}\left(\phi_{1}^{+} \phi_{2}^{+} \psi_{1}^{+} \psi_{2}^{+}\right)} \\
\quad \times\left[\phi_{12}^{+}\left(d_{22}(\lambda) \psi_{21}^{+}-\lambda^{-3 / 2} \phi_{21}^{+}\right)-\phi_{11}^{+}\left(d_{22}(\lambda) \psi_{22}^{+}-\lambda^{-3 / 2} \phi_{22}^{+}\right)\right]+\mathbf{O}(|\lambda|) .
\end{aligned}
$$

Combining (ii) with the estimate

$$
\begin{aligned}
& \operatorname{det}\left(\begin{array}{llll}
\phi_{1}^{+} & \phi_{2}^{+} & \psi_{1}^{+} & \psi_{2}^{+}
\end{array}\right) \\
& =e^{\int_{K}^{x} \mu_{1}(s ; \lambda)+\mu_{3}(s ; \lambda) d s}\left(\operatorname{ord}\left(\lambda^{3 / 2}\right) \operatorname{ord}\left(|x|^{-2}\right)+\operatorname{ord}(\lambda) \operatorname{ord}\left(|x|^{-3}\right) \widetilde{E}\right)
\end{aligned}
$$

and repeating the analysis for $\tilde{\phi}_{22}^{+}$and $\tilde{\psi}_{22}^{+}$, we obtain (iii). 
Finally, for estimate (iv) we note that

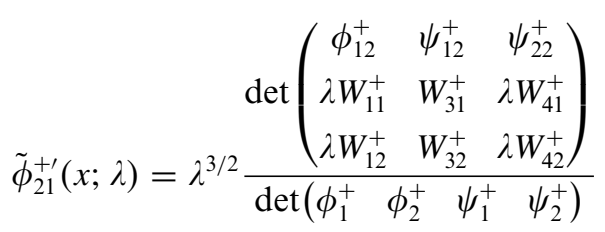

$$
\begin{aligned}
& =\frac{\lambda^{3 / 2}}{\operatorname{det}\left(\phi_{1}^{+} \quad \phi_{2}^{+} \quad \psi_{1}^{+} \quad \psi_{2}^{+}\right)} \\
& \times\left[\left(\lambda \phi_{12}^{+} W_{42}^{+}-\lambda \psi_{22}^{+} W_{12}^{+}\right) W_{31}^{+}+\left(\lambda \psi_{22}^{+} W_{11}^{+}-\lambda \phi_{12}^{+} W_{41}^{+}\right) W_{32}^{+}+\mathbf{O}\left(\left|\lambda^{2}\right|\right)\right],
\end{aligned}
$$

with also

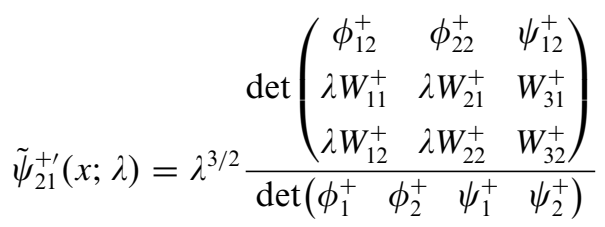

$$
\begin{aligned}
& =\frac{\lambda^{3 / 2}}{\operatorname{det}\left(\begin{array}{llll}
\phi_{1}^{+} & \phi_{2}^{+} & \psi_{1}^{+} & \psi_{2}^{+}
\end{array}\right)} \\
& \times\left[\left(\lambda \phi_{12}^{+} W_{21}^{+}-\lambda \phi_{22}^{+} W_{11}^{+}\right) W_{32}^{+}+\left(\lambda \phi_{22}^{+} W_{12}^{+}-\lambda \phi_{12}^{+} W_{22}^{+}\right) W_{31}^{+}+\mathbf{O}\left(\left|\lambda^{2}\right|\right)\right] .
\end{aligned}
$$

Combining, we have

$$
\begin{aligned}
d_{22}^{+}(\lambda) \tilde{\psi}_{2}^{+\prime}(x ; \lambda)+\lambda^{-3 / 2} \tilde{\phi}_{2}^{+\prime}(x ; \lambda)= & \frac{\lambda^{5 / 2} W_{31}^{+}}{\operatorname{det}\left(\phi_{1}^{+} \quad \phi_{2}^{+} \quad \psi_{1}^{+} \quad \psi_{2}^{+}\right)}\left[d_{22}(\lambda)\left(\phi_{22}^{+} W_{12}^{+}-\phi_{12}^{+} W_{22}^{+}\right)\right. \\
& \left.+\lambda^{-3 / 2}\left(\phi_{12}^{+} W_{42}^{+}-\psi_{22}^{+} W_{12}^{+}\right)+\mathbf{O}(|\lambda|)\right] \\
& -\frac{\lambda^{5 / 2} W_{31}^{+}}{\operatorname{det}\left(\phi_{1}^{+} \quad \phi_{2}^{+} \psi_{1}^{+} \quad \psi_{2}^{+}\right)}\left[d_{22}(\lambda)\left(\phi_{12}^{+} W_{21}^{+}-\phi_{22}^{+} W_{11}^{+}\right)\right. \\
& \left.+\lambda^{-3 / 2}\left(\psi_{22}^{+} W_{11}^{+}-\phi_{12}^{+} W_{41}^{+}\right)+\mathbf{O}(|\lambda|)\right] \\
= & \frac{\lambda^{5 / 2} W_{31}^{+}}{\operatorname{det}\left(\phi_{1}^{+} \phi_{2}^{+} \psi_{1}^{+} \psi_{2}^{+}\right)}\left[W_{12}^{+}\left(d_{22}(\lambda) \phi_{22}^{+}-\lambda^{-3 / 2} \psi_{22}^{+}\right)\right. \\
& \left.-\phi_{12}^{+}\left(d_{22}(\lambda) W_{22}^{+}-\lambda^{-3 / 2} W_{42}^{+}\right)+\mathbf{O}(|\lambda|)\right] \\
& -\frac{\lambda^{5 / 2} W_{32}^{+}}{\operatorname{det}\left(\phi_{1}^{+} \phi_{2}^{+} \psi_{1}^{+} \quad \psi_{2}^{+}\right)}\left[W_{11}^{+}\left(d_{22}(\lambda) \phi_{22}^{+}-\lambda^{-3 / 2} \psi_{22}^{+}\right)\right. \\
& \left.-\phi_{12}^{+}\left(d_{22}(\lambda) W_{21}^{+}-\lambda^{-3 / 2} W_{41}^{+}\right)+\mathbf{O}(|\lambda|)\right],
\end{aligned}
$$

from which we have (iv).

\section{Estimates on $G_{\lambda}(x ; y)$}

In this section we develop estimates on $G_{\lambda}(x, y)$, first for the critical case of $\lambda$ near zero (which will correspond with large $t$ behavior). 
Lemma 3.1 (Small $|\lambda| G_{\lambda}(x, y)$ estimates). Under the assumptions of Theorem 1.1, there exists $r$ sufficiently small so that for $|\lambda| \leq r$, and for some constant $K$ sufficiently large, we have the following estimates, for which terms containing $\mathbf{O}_{a}(\cdot)$ are meromorphic to the right of $\Gamma_{d}$, while the remaining terms are analytic to the right of $\Gamma_{d}$ and away from the negative real axis.

(i) $y \leq x \leq 0$ :

$$
\begin{aligned}
G_{\lambda}(x, y)= & \mathbf{O}_{a}(1) e^{\mu_{2}^{-}(\lambda)(x-y)}+\mathbf{O}_{a}(1) e^{\mu_{3}^{-}(\lambda) x-\mu_{2}^{-}(\lambda) y}+\mathbf{O}\left(\left|\lambda^{1 / 2} \ln \lambda\right|\right) e^{\mu_{3}^{-}(\lambda) x-\mu_{2}^{-}(\lambda) y} \\
& +\bar{u}_{x}(x)\left(\mathbf{O}_{a}\left(\left|\lambda^{-1}\right|\right)+\mathbf{O}\left(\left|\lambda^{-1 / 2} \ln \lambda\right|\right)\right) e^{-\mu_{2}^{-}(\lambda) y} \\
\partial_{y} G_{\lambda}(x, y)= & \mathbf{O}_{a}(|\lambda|) e^{\mu_{2}^{-}(\lambda)(x-y)}+\mathbf{O}_{a}(|\lambda|) e^{\mu_{3}^{-}(\lambda) x-\mu_{2}^{-}(\lambda) y}+\mathbf{O}_{a}(1) e^{\mu_{1}^{-}(\lambda)(x-y)} \\
& +\mathbf{O}\left(\left|\lambda^{3 / 2} \ln \lambda\right|\right) e^{\mu_{3}^{-}(\lambda) x-\mu_{2}^{-}(\lambda) y}+\bar{u}_{x}(x)\left(\mathbf{O}_{a}(1)+\mathbf{O}\left(\left|\lambda^{1 / 2} \ln \lambda\right|\right)\right) e^{-\mu_{2}^{-}(\lambda) y} .
\end{aligned}
$$

(ii) $x \leq y \leq 0$ :

$$
\begin{aligned}
G_{\lambda}(x, y)= & \mathbf{O}_{a}(1) e^{\mu_{3}^{-}(\lambda)(x-y)}+\mathbf{O}_{a}(1) e^{\mu_{3}^{-}(\lambda) x-\mu_{2}^{-}(\lambda) y}+\mathbf{O}\left(\left|\lambda^{1 / 2} \ln \lambda\right|\right) e^{\mu_{3}^{-}(\lambda) x-\mu_{2}^{-}(\lambda) y} \\
& +\bar{u}_{x}(x)\left(\mathbf{O}_{a}\left(\left|\lambda^{-1}\right|\right)+\mathbf{O}\left(\left|\lambda^{-1 / 2} \ln \lambda\right|\right)\right) e^{-\mu_{2}^{-}(\lambda) y} \\
\partial_{y} G_{\lambda}(x, y)= & \mathbf{O}_{a}(|\lambda|) e^{\mu_{3}^{-}(\lambda)(x-y)}+\mathbf{O}_{a}(|\lambda|) e^{\mu_{3}^{-}(\lambda) x-\mu_{2}^{-}(\lambda) y}+\mathbf{O}_{a}(1) e^{\mu_{4}^{-}(\lambda)(x-y)} \\
& +\mathbf{O}\left(\left|\lambda^{3 / 2} \ln \lambda\right|\right) e^{\mu_{3}^{-}(\lambda) x-\mu_{2}^{-}(\lambda) y}+\bar{u}_{x}(x)\left(\mathbf{O}_{a}(1)+\mathbf{O}\left(\left|\lambda^{1 / 2} \ln \lambda\right|\right)\right) e^{-\mu_{2}^{-}(\lambda) y} .
\end{aligned}
$$

(iii) $x \leq 0<K \leq y$ :

$$
\begin{aligned}
G_{\lambda}(x, y)= & \mathbf{O}_{a}(1) e^{\mu_{3}^{-}(\lambda) x-\int_{K}^{y} \mu_{3}(s ; \lambda) d s}+\mathbf{O}\left(\left|\lambda^{1 / 2} \ln \lambda\right|\right) e^{\mu_{3}^{-}(\lambda) x-\int_{K}^{y} \mu_{3}(s ; \lambda) d s} \\
& +\mathbf{O}(1) e^{\mu_{3}^{-}(\lambda) x-\sqrt{\lambda} y}+\bar{u}_{x}(x)\left(\mathbf{O}_{a}\left(\left|\lambda^{-1}\right|\right)+\mathbf{O}\left(\left|\lambda^{-1 / 2} \ln \lambda\right|\right)\right) e^{-\int_{K}^{y} \mu_{3}(s ; \lambda) d s} \\
& +\bar{u}_{x}(x)\left(\mathbf{O}_{a}\left(\left|\lambda^{-1}\right|\right)+\mathbf{O}\left(\left|\lambda^{-1 / 2} \ln \lambda\right|\right)\right) e^{-\sqrt{\lambda} y}, \\
\partial_{y} G_{\lambda}(x, y)= & \mathbf{O}_{a}(|\lambda|) e^{\mu_{3}^{-}(\lambda) x-\int_{K}^{y} \mu_{3}(s ; \lambda) d s}+\mathbf{O}\left(\left|\lambda^{3 / 2} \ln \lambda\right|\right) e^{\mu_{3}^{-}(\lambda) x-\int_{K}^{y} \mu_{3}(s ; \lambda) d s} \\
& +\mathbf{O}(|\lambda|) \mathbf{O}(|y|) e^{\mu_{3}^{-}(\lambda) x-\sqrt{\lambda} y}+\bar{u}_{x}(x)\left(\mathbf{O}_{a}(1)+\mathbf{O}\left(\left|\lambda^{1 / 2} \ln \lambda\right|\right)\right) e^{-\int_{K}^{y} \mu_{3}(s ; \lambda) d s} \\
& +\bar{u}_{x}(x) \mathbf{O}(|y|) e^{-\sqrt{\lambda} y} .
\end{aligned}
$$

(iv) $y \leq 0<K \leq x$ :

$$
\begin{aligned}
G_{\lambda}(x, y)= & \mathbf{O}\left(\left|\lambda^{-1 / 2}\right|\right) \mathbf{O}\left(|x|^{-1}\right) e^{-\sqrt{\lambda} x-\mu_{2}^{-}(\lambda) y}+\mathbf{O}\left(\left|\lambda^{-1 / 2} \ln \lambda\right|\right) \mathbf{O}\left(|x|^{-2}\right) e^{-\sqrt{\lambda} x-\mu_{2}^{-}(\lambda) y} \\
& +\bar{u}_{x}(x) \mathbf{O}_{a}\left(\left|\lambda^{-1}\right|\right) e^{-\mu_{2}^{-}(\lambda) y} \\
\partial_{y} G_{\lambda}(x, y)= & \mathbf{O}\left(\left|\lambda^{1 / 2}\right|\right) \mathbf{O}\left(|x|^{-1}\right) e^{-\sqrt{\lambda} x-\mu_{2}^{-}(\lambda) y}+\mathbf{O}\left(\left|\lambda^{1 / 2} \ln \lambda\right|\right) e^{\sqrt{\lambda} x-\mu_{2}^{-}(\lambda) y} \\
& +\bar{u}_{x}(x) \mathbf{O}_{a}(1) e^{-\mu_{2}^{-}(\lambda) y} .
\end{aligned}
$$

(v) $0<K \leq y \leq x$ :

$$
\begin{aligned}
& G_{\lambda}(x, y) \\
& \begin{aligned}
=e^{-\sqrt{\lambda}|x-y|}[ & \mathbf{O}\left(\mid \lambda^{-1 / 2}\right) \mathbf{O}\left(|x|^{-1}\right) \mathbf{O}(|y|)+\bar{u}_{x}(x) \mathbf{O}_{a}\left(\left|\lambda^{-1}\right|\right) \\
& \left.+\mathbf{O}\left(\left|\lambda^{-1 / 2}(\ln \lambda)^{2}\right|\right) \mathbf{O}\left(|x|^{-2}\right) \mathbf{O}(|y|)\right]
\end{aligned}
\end{aligned}
$$




$$
\begin{aligned}
& +e^{-\sqrt{\lambda} x-\int_{K}^{y} \mu_{3}(s ; \lambda) d s}\left[\mathbf{O}\left(\left|\lambda^{-1 / 2}\right|\right) \mathbf{O}\left(|x|^{-1}\right)+\mathbf{O}\left(|x|^{-2}\right) \mathbf{O}\left(\left|\lambda^{-1 / 2} \ln \lambda\right|\right)\right] \\
& +\mathbf{O}_{a}(1) e^{\int_{y}^{x} \mu_{1}(s ; \lambda) d s}+\bar{u}_{x}(x) \mathbf{O}_{a}\left(\left|\lambda^{-1}\right|\right) e^{-\int_{K}^{y} \mu_{3}(s ; \lambda) d s}, \\
\partial_{y} G_{\lambda}(x, y) & \\
= & e^{-\sqrt{\lambda}|x-y|}\left[\mathbf{O}\left(|x|^{-2}\right) \mathbf{O}(|y|)+\mathbf{O}\left(\left|\lambda^{1 / 2} \ln \lambda\right|\right) \mathbf{O}\left(|x|^{-1}\right)+\mathbf{O}\left(\left|\lambda^{1 / 2}\right|\right) \mathbf{O}\left(|x|^{-1}\right) \mathbf{O}(|y|)\right. \\
& \left.+\mathbf{O}(|\ln \lambda|) \mathbf{O}\left(|x|^{-2}\right)\right]+\mathbf{O}\left(\left|\lambda^{1 / 2}\right|\right) \mathbf{O}\left(|x|^{-1}\right) e^{-\sqrt{\lambda} x-\int_{K}^{y} \mu_{3}(s ; \lambda) d s} \\
& +\bar{u}_{x}(x) \mathbf{O}_{a}(1) e^{-\int_{K}^{y} \mu_{3}(s ; \lambda) d s}+\mathbf{O}_{a}(1) e^{\int_{y}^{x} \mu_{1}(s ; \lambda) d s}+\mathbf{O}\left(\left|\lambda^{1 / 2} \ln \lambda\right|\right) e^{\int_{y}^{x} \mu_{1}(s ; \lambda) d s} .
\end{aligned}
$$

(vi) $0<K \leq x \leq y$ :

$$
\begin{aligned}
G_{\lambda}(x, y)= & e^{-\sqrt{\lambda}|x-y|}\left[\mathbf{O}\left(\left|\lambda^{-1 / 2}\right|\right) \mathbf{O}\left(|x|^{-1}\right) \mathbf{O}(|y|)+\bar{u}_{x}(x) \mathbf{O}_{a}\left(\left|\lambda^{-1}\right|\right) \mathbf{O}\left(|x|^{-2}\right)\right. \\
& \left.+\mathbf{O}\left(\left|\lambda^{-1 / 2}(\ln \lambda)^{2}\right|\right) \mathbf{O}\left(|x|^{-1}\right)\right]+e^{-\int_{x}^{y} \mu_{3}(s ; \lambda) d s}\left[\mathbf{O}_{a}(1)+\mathbf{O}\left(\left|\lambda^{1 / 2} \ln \lambda\right|\right)\right] \\
& +e^{-\sqrt{\lambda} x-\int_{K}^{y} \mu_{3}(s ; \lambda) d s}\left[\mathbf{O}\left(\left|\lambda^{-1 / 2}\right|\right) \mathbf{O}\left(|x|^{-1}\right)+\mathbf{O}\left(|x|^{-2}\right) \mathbf{O}\left(\left|\lambda^{-1 / 2} \ln \lambda\right|\right)\right] \\
& +\bar{u}_{x}(x) \mathbf{O}_{a}\left(\left|\lambda^{-1}\right|\right) e^{-\int_{K}^{y} \mu_{3}(s ; \lambda) d s}, \\
\partial_{y} G_{\lambda}(x, y)= & e^{-\sqrt{\lambda}|x-y|} \mathbf{O}\left(|x|^{-1}\right) \mathbf{O}(|y|)+\mathbf{O}_{a}(|\lambda|) e^{-\int_{x}^{y} \mu_{3}(s ; \lambda) d s} \\
& +\mathbf{O}\left(\left|\lambda^{1 / 2}\right|\right) \mathbf{O}\left(|x|^{-1}\right) e^{-\sqrt{\lambda} x-\int_{K}^{y} \mu_{3}(s ; \lambda) d s}+\bar{u}_{x}(x) \mathbf{O}_{a}(1) e^{-\int_{K}^{y} \mu_{3}(s ; \lambda) d s} \\
& +\mathbf{O}\left(\left|\lambda^{3 / 2} \ln \lambda\right|\right) e^{-\int_{x}^{y} \mu_{3}(s ; \lambda) d s} .
\end{aligned}
$$

For $0 \leq y \leq K$, the expressions $e^{\int_{K}^{y} \mu_{3}(s ; \lambda) d s}$ can be replaced by $\mathbf{O}(1)$ and similarly for $x$.

Remarks on Lemma 3.1. The fundamentally new aspect of the estimates of Lemma 3.1 with respect to analogous results for nondegenerate waves is the loss of analyticity in a neighborhood of $\lambda=0$. On the degenerate side $(x>0)$, our linear equation behaves like a heat equation, and we expect such loss of analyticity, at least with terms of the form $\sqrt{\lambda}$. Even on the nondegenerate side, however, the Green's function is constructed from solutions that decay at both $\pm \infty$ and our expansion coefficients carry $\sqrt{\lambda}$ and $\sqrt{\lambda} \ln \lambda$ behavior.

Proof of Lemma 3.1. Following Zumbrun and Howard (1998/2002), we establish estimates on $G_{\lambda}(x, y)$ through the useful representation

$$
\left(\begin{array}{cc}
G_{\lambda} & G_{\lambda_{y}} \\
G_{\lambda_{x}} & G_{\lambda_{x y}}
\end{array}\right)= \begin{cases}\Phi^{+}(x ; \lambda) M^{+}(\lambda) \widetilde{\Psi}^{-}(y ; \lambda), & x>y \\
\Phi^{-}(x ; \lambda) M^{-}(\lambda) \widetilde{\Psi}^{+}(y ; \lambda), & x<y,\end{cases}
$$

where

$$
\Phi^{ \pm}=\left(\begin{array}{cc}
\Phi_{1}^{ \pm} & \Phi_{2}^{ \pm} \\
\Phi_{1}^{ \pm \prime} & \Phi_{2}^{ \pm \prime}
\end{array}\right)
$$

denote solutions to the eigenvalue $\operatorname{ODE}(2.1)$, while

$$
\widetilde{\Psi}^{ \pm}=\left(\begin{array}{cc}
\widetilde{\Psi}_{1}^{ \pm} & \widetilde{\Psi}_{1}^{ \pm \prime} \\
\widetilde{\Psi}_{2}^{ \pm} & \widetilde{\Psi}_{2}^{ \pm \prime}
\end{array}\right)
$$


denote solutions to the dual ODE (2.4). According to Zumbrun and Howard $(1998 / 2002)$ and the methods described there, we have the additional representations,

$$
\left(\begin{array}{ll}
G_{\lambda} & G_{\lambda_{y}} \\
G_{\lambda_{x}} & G_{\lambda_{x y}}
\end{array}\right)= \begin{cases}-\left(0, \Phi^{-}(x ; \lambda)\right)\left(\Phi^{+}(y ; \lambda), \Phi^{-}(y ; \lambda)\right)^{-1} S(y)^{-1}, & x \leq y \\
\left(\Phi^{+}(x ; \lambda), 0\right)\left(\Phi^{+}(y ; \lambda), \Phi^{-}(y ; \lambda)\right)^{-1} S(y)^{-1}, & x \geq y\end{cases}
$$

and

$$
\left(\begin{array}{cc}
G_{\lambda} & G_{\lambda_{y}} \\
G_{\lambda_{x}} & G_{\lambda_{x y}}
\end{array}\right)=\left\{\begin{array}{cc}
-S(x)^{-1}\left(\begin{array}{c}
\widetilde{\Psi}^{-}(x ; \lambda) \\
\widetilde{\Psi}^{+}(x ; \lambda)
\end{array}\right)^{-1}\left(\begin{array}{c}
0 \\
\widetilde{\Psi}^{+}(y ; \lambda)
\end{array}\right), & x \leq y \\
S(x)^{-1}\left(\begin{array}{c}
\widetilde{\Psi}^{-}(x ; \lambda) \\
\tilde{\Psi}^{+}(x ; \lambda)
\end{array}\right)^{-1}\left(\begin{array}{c}
\widetilde{\Psi}^{-}(y ; \lambda) \\
0
\end{array}\right), & x \geq y .
\end{array}\right.
$$

In order to see the validity of these relationships, we briefly recall the development of Zumbrun and Howard (1998/2002). Observing the jump condition,

$$
\left[\begin{array}{cc}
G_{\lambda} & G_{\lambda_{y}} \\
G_{\lambda_{x}} & G_{\lambda_{x y}}
\end{array}\right]=\left(\begin{array}{cc}
0 & -I \\
I & -A
\end{array}\right)=: S^{-1}
$$

we have

$$
\Phi^{+}(y ; \lambda) M^{+}(\lambda) \widetilde{\Psi}^{+}(y ; \lambda)-\Phi^{-}(y ; \lambda) M^{-}(\lambda) \widetilde{\Psi}^{+}(y ; \lambda)=S(y)^{-1}
$$

or in matrix form

$$
\left(\Phi^{+}(y ; \lambda), \Phi^{-}(y ; \lambda)\right)\left(\begin{array}{cc}
M^{+}(\lambda) & 0 \\
0 & -M^{-}(\lambda)
\end{array}\right)\left(\begin{array}{l}
\widetilde{\Psi}^{-}(y ; \lambda) \\
\widetilde{\Psi}^{+}(y ; \lambda)
\end{array}\right)=S(y)^{-1},
$$

from which we conclude the critical relation

$$
\left(\begin{array}{cc}
M^{+}(\lambda) & 0 \\
0 & -M^{-}(\lambda)
\end{array}\right)=\left(\Phi^{+}(y ; \lambda), \Phi^{-}(y ; \lambda)\right)^{-1} S(y)^{-1}\left(\begin{array}{c}
\tilde{\Psi}^{-}(y ; \lambda) \\
\tilde{\Psi}^{+}(y ; \lambda)
\end{array}\right)^{-1}
$$

In order to establish (3.1), we rewrite this last relationship as

$$
\left(\Phi^{+}(y ; \lambda), \Phi^{-}(y ; \lambda)\right)^{-1} S(y)^{-1}=\left(\begin{array}{cc}
M^{+}(\lambda) & 0 \\
0 & -M^{-}(\lambda)
\end{array}\right)\left(\begin{array}{l}
\widetilde{\Psi}^{-}(y ; \lambda) \\
\widetilde{\Psi}^{+}(y ; \lambda)
\end{array}\right),
$$

and for $x>y$ multiply on both sides by $\left(\Phi^{+}(x ; \lambda), 0\right)$ and for $x<y$ multiply on both sides by $-\left(0, \Phi^{-}(y ; \lambda)\right)$. The case of (3.2) is similar.

Case (i) $y \leq x \leq 0$. In the case $y \leq x \leq 0$, we compare representations

$$
\left(\begin{array}{cc}
G_{\lambda} & G_{\lambda_{y}} \\
G_{\lambda_{x}} & G_{\lambda_{x y}}
\end{array}\right)=\sum_{j, k=1}^{2} \phi_{k}^{-}(x ; \lambda) d_{k j}^{-}(\lambda) \tilde{\psi}_{j}^{-}(y ; \lambda)+\sum_{j, k=1}^{2} \psi_{k}^{-}(x ; \lambda) e_{k j}^{-}(\lambda) \tilde{\psi}_{j}^{-}(y ; \lambda)
$$


and

$$
\begin{aligned}
\left(\begin{array}{cc}
G_{\lambda} & G_{\lambda_{y}} \\
G_{\lambda_{x}} & G_{\lambda_{x y}}
\end{array}\right) & =S(x)^{-1}\left(\begin{array}{c}
\widetilde{\Psi}^{-}(x ; \lambda) \\
\widetilde{\Psi}^{+}(x ; \lambda)
\end{array}\right)^{-1}\left(\begin{array}{c}
\widetilde{\Psi}^{-}(y ; \lambda) \\
0
\end{array}\right) \\
& =\sum_{j=1}^{2} S(x)^{-1}\left(\begin{array}{c}
\widetilde{\Psi}^{-}(x ; \lambda) \\
\tilde{\Psi}^{+}(x ; \lambda)
\end{array}\right)^{-1} E_{j} \tilde{\psi}_{j}^{-}(y ; \lambda)
\end{aligned}
$$

to obtain the relation

$$
\sum_{k=1}^{2} \phi_{k}^{-}(x ; \lambda) d_{k j}^{-}(\lambda)+\sum_{k=1}^{2} \psi_{k}^{-}(x ; \lambda) e_{k j}^{-}(\lambda)=S(x)^{-1}\left(\begin{array}{c}
\tilde{\Psi}^{-}(x ; \lambda) \\
\tilde{\Psi}^{+}(x ; \lambda)
\end{array}\right)^{-1} E_{j}
$$

Multiplying (3.3) on the left by $\tilde{\psi}_{l}^{-}(x ; \lambda) S(x)$, and recalling relations $(2.7)$, we find

$$
e_{l j}^{-}(\lambda)=\tilde{\psi}_{l}^{-}(x ; \lambda)\left(\begin{array}{c}
\tilde{\Psi}^{-}(x ; \lambda) \\
\tilde{\Psi}^{+}(x ; \lambda)
\end{array}\right)^{-1} E_{j}
$$

for which $e_{l j}^{-}(\lambda)$ is the $j$ th component of the vector $J$ satisfying

$$
\left(\begin{array}{c}
\widetilde{\Psi}^{-}(x ; \lambda) \\
\widetilde{\Psi}^{+}(x ; \lambda)
\end{array}\right) J=\tilde{\psi}_{l}^{-}(x ; \lambda) .
$$

Proceeding by Cramer's rule, we have for $j=1$

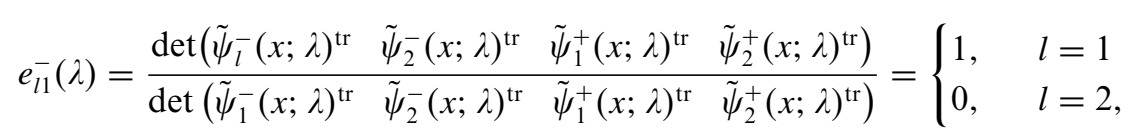

and similarly for $j=2$, so that $e_{l j}^{-}(\lambda)=\delta_{l}^{j}$, where $\delta_{l}^{j}$ represents the Kronecker delta.

Alternatively, multiplying (3.3) on the left by $\tilde{\phi}_{l}^{-}(x ; \lambda) S(x)$ we find

$$
d_{l j}^{-}(\lambda)=\tilde{\phi}_{l}^{-}(x ; \lambda)\left(\begin{array}{l}
\tilde{\Psi}^{-}(x ; \lambda) \\
\tilde{\Psi}^{+}(x ; \lambda)
\end{array}\right)^{-1} E_{j}
$$

Proceeding again by Cramer's rule and observing the estimates of Lemma 2.5 $\widetilde{\Phi}_{1}^{-\prime}(x ; \lambda)=\mathbf{O}(|\lambda|)$ and $\widetilde{\Psi}_{2}^{-1}(x ; \lambda)=\mathbf{O}(|\lambda|)$, we find the estimates $d_{11}^{-}(\lambda)=\mathbf{O}_{a}(|\lambda|)+$ $\mathbf{O}\left(\left|\lambda^{3 / 2} \ln \lambda\right|\right), \quad d_{12}^{-}(\lambda)=\mathbf{O}_{a}(1)+\mathbf{O}\left(\left|\lambda^{1 / 2} \ln \lambda\right|\right), \quad d_{21}(\lambda)=\mathbf{O}_{a}(1)+\mathbf{O}\left(\left|\lambda^{1 / 2} \ln \lambda\right|\right), \quad$ and $d_{22}^{-}(\lambda)=\mathbf{O}_{a}\left(\left|\lambda^{-1}\right|\right)+\mathbf{O}\left(\left|\lambda^{-1 / 2} \ln \lambda\right|\right)$. Collecting these observations, we have

$$
\begin{aligned}
& \left(\begin{array}{c}
\Psi_{1}^{-}(x ; \lambda) \widetilde{\Psi}_{1}^{-}(y ; \lambda) \\
\Psi_{1}^{-}(x ; \lambda) \widetilde{\Psi}_{1}^{-\prime}(y ; \lambda)
\end{array}\right)=\left(\begin{array}{c}
\mathbf{O}_{a}(1) e^{\mu_{1}^{-}(\lambda)(x-y)} \\
\mathbf{O}_{a}(1) e^{\mu_{1}^{-}(\lambda)(x-y)}
\end{array}\right), \\
& \left(\begin{array}{c}
\Psi_{2}^{-}(x ; \lambda) \widetilde{\Psi}_{2}^{-}(y ; \lambda) \\
\Psi_{2}^{-}(x ; \lambda) \widetilde{\Psi}_{2}^{-\prime}(y ; \lambda)
\end{array}\right)=\left(\begin{array}{c}
\mathbf{O}_{a}(1) e^{\mu_{2}^{-}(\lambda)(x-y)} \\
\mathbf{O}_{a}(|\lambda|) e^{\mu_{2}^{-}(\lambda)(x-y)}
\end{array}\right),
\end{aligned}
$$




$$
\begin{aligned}
\left(\begin{array}{c}
\Phi_{1}^{-}(x ; \lambda) d_{11}^{-}(\lambda) \widetilde{\Psi}_{1}^{-}(y ; \lambda) \\
\Phi_{1}^{-}(x ; \lambda) d_{11}^{-}(\lambda) \widetilde{\Psi}_{1}^{-\prime}(y ; \lambda)
\end{array}\right)=\left(\begin{array}{c}
\left(\mathbf{O}_{a}(|\lambda|)+\mathbf{O}\left(\left|\lambda^{3 / 2} \ln \lambda\right|\right)\right) e^{\mu_{3}^{-}(\lambda) x-\mu_{1}^{-}(\lambda) y} \\
\left(\mathbf{O}_{a}(|\lambda|)+\mathbf{O}\left(\left|\lambda^{3 / 2} \ln \lambda\right|\right)\right) e^{\mu_{3}^{-}(\lambda) x-\mu_{1}^{-}(\lambda) y}
\end{array}\right) \\
\left(\begin{array}{c}
\Phi_{1}^{-}(x ; \lambda) d_{12}^{-}(\lambda) \widetilde{\Psi}_{2}^{-}(y ; \lambda) \\
\Phi_{1}^{-}(x ; \lambda) d_{12}^{-}(\lambda) \widetilde{\Psi}_{2}^{-\prime}(y ; \lambda)
\end{array}\right)=\left(\begin{array}{c}
\left(\mathbf{O}_{a}(1)+\mathbf{O}\left(\left|\lambda^{1 / 2} \ln \lambda\right|\right)\right) e^{\mu_{3}^{-}(\lambda) x-\mu_{2}^{-}(\lambda) y} \\
\left(\mathbf{O}_{a}(|\lambda|)+\mathbf{O}\left(\left|\lambda^{3 / 2} \ln \lambda\right|\right)\right) e^{\mu_{3}^{-}(\lambda) x-\mu_{2}^{-}(\lambda) y}
\end{array}\right) \\
\left(\begin{array}{c}
\Phi_{2}^{-}(x ; \lambda) d_{21}^{-}(\lambda) \widetilde{\Psi}_{1}^{-}(y ; \lambda) \\
\Phi_{2}^{-}(x ; \lambda) d_{21}^{-}(\lambda) \widetilde{\Psi}_{1}^{-\prime}(y ; \lambda)
\end{array}\right)=\left(\begin{array}{c}
\left(\mathbf{O}_{a}(1)+\mathbf{O}\left(\left|\lambda^{1 / 2} \ln \lambda\right|\right)\right) e^{\mu_{4}^{-}(\lambda) x-\mu_{1}^{-}(\lambda) y} \\
\left(\mathbf{O}_{a}(1)+\mathbf{O}\left(\left|\lambda^{1 / 2} \ln \lambda\right|\right)\right) e^{\mu_{4}^{-}(\lambda) x-\mu_{1}^{-}(\lambda) y}
\end{array}\right) \\
\left(\begin{array}{c}
\Phi_{2}^{-}(x ; \lambda) d_{22}^{-}(\lambda) \widetilde{\Psi}_{2}^{-}(y ; \lambda) \\
\Phi_{2}^{-}(x ; \lambda) d_{22}^{-}(\lambda) \widetilde{\Psi}_{2}^{-\prime}(y ; \lambda)
\end{array}\right)=\left(\begin{array}{c}
\left(\mathbf{O}_{a}\left(\left|\lambda^{-1}\right|\right)+\mathbf{O}\left(\left|\lambda^{-1 / 2} \ln \lambda\right|\right)\right) e^{\mu_{4}^{-}(\lambda) x-\mu_{2}^{-}(\lambda) y} \\
\left(\mathbf{O}_{a}(1)+\mathbf{O}\left(\left|\lambda^{1 / 2} \ln \lambda\right|\right)\right) e^{\mu_{4}^{-}(\lambda) x-\mu_{2}^{-}(\lambda) y}
\end{array}\right) .
\end{aligned}
$$

Finally, observing that $\Phi_{1}^{-}(x ; 0)$ does not decay as $x \rightarrow-\infty$, we conclude that

$$
\Phi_{2}^{-}(x ; 0)=P \bar{u}_{x}(x),
$$

for some projection constant $P$, from which the estimates of Case (i) are immediate.

Case (ii) $x \leq y \leq 0$. The analysis of Case (ii) is almost identical to the analysis of Case (i). The only new terms are $\Phi_{1}^{-}(x ; \lambda) \widetilde{\Phi}_{1}^{-}(x ; \lambda)$ and $\Phi_{2}^{-}(x ; \lambda) \widetilde{\Phi}_{2}^{-}(x ; \lambda)$, for which we find

$$
\begin{aligned}
& \left(\begin{array}{c}
\Phi_{1}^{-}(x ; \lambda) \widetilde{\Phi}_{1}^{-}(y ; \lambda) \\
\Phi_{1}^{-}(x ; \lambda) \widetilde{\Phi}_{1}^{-\prime}(y ; \lambda)
\end{array}\right)=\left(\begin{array}{c}
\mathbf{O}_{a}(1) e^{\mu_{3}^{-}(\lambda)(x-y)} \\
\mathbf{O}_{a}(|\lambda|) e^{\mu_{3}^{-}(\lambda)(x-y)}
\end{array}\right), \\
& \left(\begin{array}{c}
\Phi_{2}^{-}(x ; \lambda) \widetilde{\Phi}_{2}^{-}(y ; \lambda) \\
\Phi_{2}^{-}(x ; \lambda) \widetilde{\Phi}_{2}^{-\prime}(y ; \lambda)
\end{array}\right)=\left(\begin{array}{l}
\mathbf{O}_{a}(1) e^{\mu_{4}^{-}(\lambda)(x-y)} \\
\mathbf{O}_{a}(1) e^{\mu_{4}^{-}(\lambda)(x-y)}
\end{array}\right) .
\end{aligned}
$$

Case (iii) $x \leq 0<K \leq y$. In the case $x \leq 0<K \leq y$, we compare the representations

$$
\left(\begin{array}{cc}
G_{\lambda} & G_{\lambda_{y}} \\
G_{\lambda_{x}} & G_{\lambda_{x y}}
\end{array}\right)=\sum_{j, k=1}^{2} \phi_{k}^{-}(x ; \lambda) d_{k j}(\lambda) \tilde{\psi}_{j}^{+}(y ; \lambda)
$$

and

$$
\begin{aligned}
\left(\begin{array}{cc}
G_{\lambda} & G_{\lambda_{y}} \\
G_{\lambda_{x}} & G_{\lambda_{x y}}
\end{array}\right) & =-\left(0, \Phi^{-}(x ; \lambda)\right)\left(\Phi^{+}(y ; \lambda), \Phi^{-}(y ; \lambda)\right)^{-1} S(y)^{-1} \\
& =-\sum_{k=1}^{2} \phi_{k}^{-}(x ; \lambda) E_{2+k}^{\mathrm{tr}}\left(\Phi^{+}(y ; \lambda), \Phi^{-}(y ; \lambda)\right)^{-1} S(y)^{-1}
\end{aligned}
$$

to obtain the relation

$$
\sum_{j=1}^{2} d_{k j}^{-}(\lambda) \tilde{\psi}_{j}^{+}(y ; \lambda)=-E_{2+k}^{\mathrm{tr}}\left(\Phi^{+}(y ; \lambda), \Phi^{-}(y ; \lambda)\right)^{-1} S(y)^{-1} .
$$

(We observe that the scattering coefficients $d_{k j}(\lambda)$ here are not necessarily the same as the coefficients $d_{k j}^{-}(\lambda)$ from Cases (i) and (ii).) Multiplying this last equation on 
the right by $S(y) \psi_{l}^{+}(y ; \lambda)$, we find

$$
d_{k l}(\lambda) \tilde{\psi}_{l}^{+}(y ; \lambda) S(y) \psi_{l}^{+}(y ; \lambda)=-E_{2+k}^{\mathrm{tr}}\left(\Phi^{+}(y ; \lambda), \Phi^{-}(y ; \lambda)\right)^{-1} \psi_{l}^{+}(y ; \lambda) .
$$

Proceeding as in Case (i) and the proof of Lemma 2.3, we find

$$
\begin{aligned}
& d_{11}(\lambda)=\frac{\operatorname{det}\left(\phi_{1}^{+}, \phi_{2}^{+}, \psi_{1}^{+}, \phi_{2}^{-}\right)}{\operatorname{det}\left(\phi_{1}^{+}, \phi_{2}^{+}, \phi_{1}^{-}, \phi_{2}^{-}\right)}=\mathbf{O}_{a}(1)+\mathbf{O}\left(\left|\lambda^{1 / 2} \ln \lambda\right|\right) \\
& d_{21}(\lambda)=\frac{\operatorname{det}\left(\phi_{1}^{+}, \phi_{2}^{+}, \phi_{1}^{-}, \psi_{1}^{+}\right)}{\operatorname{det}\left(\phi_{1}^{+}, \phi_{2}^{+}, \phi_{1}^{-}, \phi_{2}^{-}\right)}=\mathbf{O}_{a}\left(\left|\lambda^{-1}\right|\right)+\mathbf{O}\left(\left|\lambda^{-1 / 2} \ln \lambda\right|\right) .
\end{aligned}
$$

On the other hand, for $d_{12}(\lambda)$ and $d_{22}(\lambda)$ we have, due to our scaling (2.6),

$$
\begin{aligned}
& d_{12}(\lambda)=\lambda^{-3 / 2} \frac{\operatorname{det}\left(\phi_{1}^{+}, \phi_{2}^{+}, \psi_{2}^{+}, \phi_{2}^{-}\right)}{\operatorname{det}\left(\phi_{1}^{+}, \phi_{2}^{+}, \phi_{1}^{-}, \phi_{2}^{-}\right)}, \\
& d_{21}(\lambda)=\lambda^{-3 / 2} \frac{\operatorname{det}\left(\phi_{1}^{+}, \phi_{2}^{+}, \phi_{1}^{-}, \psi_{2}^{+}\right)}{\operatorname{det}\left(\phi_{1}^{+}, \phi_{2}^{+}, \phi_{1}^{-}, \phi_{2}^{-}\right)} .
\end{aligned}
$$

In this case, we observe that the fast growth solution $\psi_{2}^{+}$can without loss of generality be constructed by determining the fast growth solution to the integrated equation (2.3) and computing its derivative. Constructed in this way, $\psi_{2}^{+}$clearly satisfies

$$
\left.\left(\Psi_{2}^{+^{\prime}}-A(x) \Psi_{2}^{+}\right)\right|_{\lambda=0}=0
$$

according to which

$$
\operatorname{det}\left(\phi_{1}^{+}, \phi_{2}^{+}, \psi_{2}^{+}, \phi_{2}^{-}\right)=\operatorname{det}\left(\begin{array}{cccc}
\Phi_{1}^{+} & \Phi_{2}^{+} & \Psi_{2}^{+} & \Phi_{2}^{-} \\
\lambda \mathscr{W}_{1}^{+} & \lambda \mathscr{W}_{2}^{+} & \lambda \mathscr{W}_{4}^{+} & \lambda \mathscr{W}_{2}^{-}
\end{array}\right) .
$$

Proceeding as in Lemma 2.3 and observing cancellation between the degenerate solutions $\Phi_{2}^{+}$and $\Psi_{2}^{+}$as in Lemma 2.6, we conclude

$$
d_{12}(\lambda)=\mathbf{O}_{a}(1)+\mathbf{O}\left(\left|\lambda^{1 / 2} \ln \lambda\right|\right)
$$

and similarly,

$$
d_{22}(\lambda)=\mathbf{O}_{a}\left(\left|\lambda^{-1}\right|\right)+\mathbf{O}\left(\left|\lambda^{-1 / 2} \ln \lambda\right|\right)
$$

Gathering these observations, we have the estimates,

$$
\begin{aligned}
\left(\begin{array}{c}
\Phi_{1}^{-}(x ; \lambda) d_{11}(\lambda) \widetilde{\Psi}_{1}^{+}(y ; \lambda) \\
\Phi_{1}^{-}(x ; \lambda) d_{11}(\lambda) \widetilde{\Psi}_{1}^{+\prime}(y ; \lambda)
\end{array}\right) & =\left(\begin{array}{c}
\left(\mathbf{O}_{a}(1)+\mathbf{O}\left(\left|\lambda^{1 / 2} \ln \lambda\right|\right)\right) e^{\mu_{3}^{-}(\lambda) x-\int_{K}^{y} \mu_{3}(s ; \lambda) d s} \\
\left(\mathbf{O}_{a}(|\lambda|)+\mathbf{O}\left(\left|\lambda^{3 / 2} \ln \lambda\right|\right)\right) e^{\mu_{3}^{-}(\lambda) x-\int_{K}^{y} \mu_{3}(s ; \lambda) d s}
\end{array}\right), \\
\left(\begin{array}{c}
\Phi_{1}^{-}(x ; \lambda) d_{12}(\lambda) \widetilde{\Psi}_{2}^{+}(y ; \lambda) \\
\Phi_{1}^{-}(x ; \lambda) d_{12}(\lambda) \widetilde{\Psi}_{2}^{+\prime}(y ; \lambda)
\end{array}\right) & =\left(\begin{array}{c}
\mathbf{O}(1) e^{\mu_{3}^{-}(\lambda) x-\sqrt{\lambda} y} \\
\mathbf{O}(|\lambda|) \mathbf{O}(|y|) e^{\mu_{3}^{-}(\lambda) x-\sqrt{\lambda} y}
\end{array}\right),
\end{aligned}
$$




$$
\begin{aligned}
& \left(\begin{array}{c}
\Phi_{2}^{-}(x ; \lambda) d_{21}(\lambda) \widetilde{\Psi}_{1}^{+}(y ; \lambda) \\
\Phi_{2}^{-}(x ; \lambda) d_{21}(\lambda) \widetilde{\Psi}_{1}^{+\prime}(y ; \lambda)
\end{array}\right)=\left(\begin{array}{c}
\left(\mathbf{O}_{a}\left(\left|\lambda^{-1}\right|\right)+\mathbf{O}\left(\left|\lambda^{-1 / 2} \ln \lambda\right|\right)\right) e^{\mu_{4}^{-}(\lambda) x-\int_{K}^{y} \mu_{3}(s ; \lambda) d s} \\
\left(\mathbf{O}_{a}(1)+\mathbf{O}\left(\left|\lambda^{1 / 2} \ln \lambda\right|\right)\right) e^{\mu_{4}^{-}(\lambda) x-\int_{K}^{y} \mu_{3}(s ; \lambda) d s}
\end{array}\right), \\
& \left(\begin{array}{c}
\Phi_{2}^{-}(x ; \lambda) d_{22}(\lambda) \widetilde{\Psi}_{2}^{+}(y ; \lambda) \\
\Phi_{2}^{-}(x ; \lambda) d_{22}(\lambda) \widetilde{\Psi}_{2}^{+\prime}(y ; \lambda)
\end{array}\right)=\left(\begin{array}{c}
\left(\mathbf{O}_{a}\left(\left|\lambda^{-1}\right|\right)+\mathbf{O}\left(\left|\lambda^{-1 / 2} \ln \lambda\right|\right)\right) e^{\mu_{4}^{-}(\lambda) x-\sqrt{\lambda} y} \\
\mathbf{O}(|y|) e^{\mu_{4}^{-}(\lambda) x-\sqrt{\lambda} y}
\end{array}\right) .
\end{aligned}
$$

Case (iv) $y \leq 0<K \leq x$. In the case $y \leq 0<K \leq x$, we compare representations

$$
\left(\begin{array}{cc}
G_{\lambda} & G_{\lambda_{y}} \\
G_{\lambda_{x}} & G_{\lambda_{x y}}
\end{array}\right)=\sum_{j, k=1}^{2} \phi_{k}^{+}(x ; \lambda) \bar{d}_{k j}(\lambda) \tilde{\psi}_{j}^{-}(y ; \lambda)
$$

and

$$
\begin{aligned}
\left(\begin{array}{cc}
G_{\lambda} & G_{\lambda_{y}} \\
G_{\lambda_{x}} & G_{\lambda_{x y}}
\end{array}\right) & =\left(\Phi^{+}(x ; \lambda), 0\right)\left(\Phi^{+}(y ; \lambda), \Phi^{-}(y ; \lambda)\right)^{-1} S(y)^{-1} \\
& =\sum_{k=1}^{2} \phi_{k}^{+}(x ; \lambda) E_{k}^{\operatorname{tr}}\left(\Phi^{+}(y ; \lambda), \Phi^{-}(y ; \lambda)\right)^{-1} S(y)^{-1},
\end{aligned}
$$

to obtain the relation

$$
\sum_{j=1}^{2} \bar{d}_{k j}(\lambda) \tilde{\psi}_{j}^{-}(y ; \lambda)=E_{k}^{\mathrm{tr}}\left(\Phi^{+}(y ; \lambda), \Phi^{-}(y ; \lambda)\right)^{-1} S(y)^{-1} .
$$

Multiplying Equation (3.5) by $S(y) \psi_{l}^{-}(y ; \lambda)$ on the right, we find

$$
\bar{d}_{k l}(\lambda)=E_{k}^{\mathrm{tr}}\left(\Phi^{+}(y ; \lambda), \Phi^{-}(y ; \lambda)\right)^{-1} \psi_{l}^{-}(y ; \lambda) .
$$

Proceeding as in Case (iii), we determine $\bar{d}_{11}(\lambda)=\mathbf{O}_{a}(1)+\mathbf{O}\left(\left|\lambda^{1 / 2} \ln \lambda\right|\right), \bar{d}_{21}(\lambda)=$ $\mathbf{O}_{a}(1)+\mathbf{O}\left(\left|\lambda^{1 / 2} \ln \lambda\right|\right)$, and $\bar{d}_{22}(\lambda)=\mathbf{O}_{a}\left(\left|\lambda^{-1}\right|\right)+\mathbf{O}\left(\left|\lambda^{-1 / 2} \ln \lambda\right|\right)$. Moreover, for the critical term $\bar{d}_{12}(\lambda)$, we have

$$
\bar{d}_{12}(\lambda)=\frac{\operatorname{det}\left(\psi_{2}^{-}, \phi_{2}^{+}, \phi_{1}^{-}, \phi_{2}^{-}\right)}{\operatorname{det}\left(\phi_{1}^{+}, \phi_{2}^{+}, \phi_{1}^{-}, \phi_{2}^{-}\right)} .
$$

According to our scaling, $\phi_{2}^{+}(x ; 0)$ and $\phi_{2}^{-}(x ; 0)$ are both proportional to $\bar{u}_{x}(x)$, and the numerator vanishes at $\lambda=0$, giving

$$
\bar{d}_{12}(\lambda)=\mathbf{O}\left(\left|\lambda^{-1 / 2} \ln \lambda\right|\right)+\mathbf{O}(|\ln \lambda|) .
$$

Collecting these observations, we find

$$
\begin{aligned}
\left(\begin{array}{c}
\Phi_{1}^{+}(x ; \lambda) \bar{d}_{11}(\lambda) \widetilde{\Psi}_{1}^{-}(y ; \lambda) \\
\Phi_{1}^{+}(x ; \lambda) \bar{d}_{11}(\lambda) \widetilde{\Psi}_{1}^{-\prime}(y ; \lambda)
\end{array}\right)=\left(\begin{array}{c}
\left(\mathbf{O}_{a}(1)+\mathbf{O}\left(\left|\lambda^{1 / 2} \ln \lambda\right|\right)\right) e^{\int_{K}^{x} \mu_{1}(s ; \lambda) d s-\mu_{1}^{-}(\lambda) y} \\
\left(\mathbf{O}_{a}(1)+\mathbf{O}\left(\left|\lambda^{1 / 2} \ln \lambda\right|\right)\right) e^{\int_{K}^{x} \mu_{1}(s ; \lambda) d s-\mu_{1}^{-}(\lambda) y}
\end{array}\right), \\
\left(\begin{array}{c}
\Phi_{1}^{+}(x ; \lambda) \bar{d}_{12}(\lambda) \widetilde{\Psi}_{2}^{-}(y ; \lambda) \\
\Phi_{1}^{+}(x ; \lambda) \bar{d}_{12}(\lambda) \widetilde{\Psi}_{2}^{-\prime}(y ; \lambda)
\end{array}\right)=\left(\begin{array}{c}
\left(\mathbf{O}\left(\left|\lambda^{-1 / 2} \ln \lambda\right|\right)+\mathbf{O}(|\ln \lambda|)\right) e^{\int_{K}^{x} \mu_{1}(s ; \lambda) d s-\mu_{2}^{-}(\lambda) y} \\
\left(\mathbf{O}_{a}(1)+\mathbf{O}\left(\left|\lambda^{1 / 2} \ln \lambda\right|\right) e^{\int_{K}^{x} \mu_{1}(s ; \lambda) d s-\mu_{2}^{-}(\lambda) y}\right.
\end{array}\right),
\end{aligned}
$$




$$
\begin{aligned}
& \left(\begin{array}{c}
\Phi_{2}^{+}(x ; \lambda) \bar{d}_{21}(\lambda) \widetilde{\Psi}_{1}^{-}(y ; \lambda) \\
\Phi_{2}^{+}(x ; \lambda) \bar{d}_{21}(\lambda) \widetilde{\Psi}_{1}^{-1}(y ; \lambda)
\end{array}\right)=\left(\begin{array}{c}
\left(\mathbf{O}\left(\left|\lambda^{1 / 2}\right|\right) \mathbf{O}\left(|x|^{-1}\right)+\mathbf{O}\left(|x|^{-2}\right)\right) e^{-\sqrt{\lambda} x-\mu_{1}^{-}(\lambda) y} \\
\left(\mathbf{O}\left(\left|\lambda^{1 / 2}\right|\right) \mathbf{O}\left(|x|^{-1}\right)+\mathbf{O}\left(|x|^{-2}\right)\right) e^{-\sqrt{\lambda} x-\mu_{1}^{-}(\lambda) y}
\end{array}\right), \\
& \left(\begin{array}{c}
\Phi_{2}^{+}(x ; \lambda) \bar{d}_{22}(\lambda) \widetilde{\Psi}_{2}^{-}(y ; \lambda) \\
\Phi_{2}^{+}(x ; \lambda) \bar{d}_{22}(\lambda) \widetilde{\Psi}_{2}^{-\prime}(y ; \lambda)
\end{array}\right) \\
& =\left(\begin{array}{c}
{\left[\mathbf{O}_{a}\left(\left|\lambda^{-1}\right|\right) \mathbf{O}\left(|x|^{-2}\right)+\mathbf{O}\left(\left|\lambda^{-1 / 2}\right|\right) \mathbf{O}\left(|x|^{-1}\right)+\mathbf{O}\left(\left|\lambda^{-1 / 2} \ln \lambda\right|\right) \mathbf{O}\left(|x|^{-2}\right)\right] e^{-\sqrt{\lambda} x-\mu_{2}^{-}(\lambda) y}} \\
{\left[\mathbf{O}\left(\left|\lambda^{1 / 2}\right|\right) \mathbf{O}\left(|x|^{-1}\right)+\mathbf{O}\left(|x|^{-2}\right)\right] e^{-\sqrt{\lambda} x-\mu_{2}^{-}(\lambda) y}}
\end{array}\right) .
\end{aligned}
$$

Case (v) $0<K \leq y \leq x$. In the case $0<K \leq y \leq x$, we compare representations

$$
\left(\begin{array}{cc}
G_{\lambda} & G_{\lambda_{y}} \\
G_{\lambda_{x}} & G_{\lambda_{x y}}
\end{array}\right)=\sum_{j, k=1}^{2} \phi_{k}^{+}(x ; \lambda) d_{k j}^{+}(\lambda) \tilde{\psi}_{j}^{+}(y ; \lambda)+\sum_{j, k=1}^{2} \phi_{k}^{+}(x ; \lambda) e_{k j}^{+}(\lambda) \tilde{\phi}_{j}^{+}(y ; \lambda)
$$

and

$$
\begin{aligned}
\left(\begin{array}{cc}
G_{\lambda} & G_{\lambda_{y}} \\
G_{\lambda_{x}} & G_{\lambda_{x y}}
\end{array}\right) & =\left(\Phi^{+}(x ; \lambda), 0\right)\left(\Phi^{+}(y ; \lambda), \Phi^{-}(y ; \lambda)\right)^{-1} S(y)^{-1} \\
& =\sum_{k=1}^{2} \phi_{k}^{+}(x ; \lambda) E_{k}^{\operatorname{tr}}\left(\Phi^{+}(y ; \lambda), \Phi^{-}(y ; \lambda)\right)^{-1} S(y)^{-1},
\end{aligned}
$$

to obtain the relation

$$
\sum_{j=1}^{2} d_{k j}^{+}(\lambda) \tilde{\psi}_{j}^{+}(y ; \lambda)+\sum_{j=1}^{2} e_{k j}^{+}(\lambda) \tilde{\phi}_{j}^{+}(y ; \lambda)=E_{k}^{\mathrm{tr}}\left(\Phi^{+}(y ; \lambda), \Phi^{-}(y ; \lambda)\right)^{-1} S(y)^{-1} .
$$

Multiplying Equation $(3.8)$ by $S(y) \phi_{l}^{+}(y ; \lambda)$ on the right and employing relations (2.6), we obtain

$$
e_{k l}^{+}(\lambda) \tilde{\phi}_{l}^{+}(y ; \lambda) S(y) \phi_{l}^{+}(y ; \lambda)=E_{k}^{\operatorname{tr}}\left(\Phi^{+}(y ; \lambda), \Phi^{-}(y ; \lambda)\right)^{-1} \phi_{l}^{+}(y ; \lambda) .
$$

In the case $k=l=2$, we have

$$
\lambda^{3 / 2} e_{22}^{+}(\lambda)=(0,1,0,0)\left(\Phi^{+}(y ; \lambda), \Phi^{-}(y ; \lambda)\right)^{-1} \phi_{2}^{+}(y ; \lambda) .
$$

Observing that $J=\left(\Phi^{+}(y ; \lambda), \Phi^{-}(y ; \lambda)\right)^{-1} \phi_{2}^{+}(y ; \lambda)$ is a matrix equation, we apply Cramer's rule to determine

$$
e_{22}^{+}(\lambda)=\lambda^{-3 / 2} J_{2}(\lambda)=\lambda^{-3 / 2} \frac{\operatorname{det}\left(\begin{array}{llll}
\phi_{1}^{+} & \phi_{2}^{+} & \phi_{1}^{-} & \phi_{2}^{-}
\end{array}\right)}{\operatorname{det}\left(\begin{array}{llll}
\phi_{1}^{+} & \phi_{2}^{+} & \phi_{1}^{-} & \phi_{2}^{-}
\end{array}\right)}=\lambda^{-3 / 2} .
$$

Similarly, $e_{12}^{+}(\lambda)=e_{21}^{+}(\lambda)=0$, and $e_{11}^{+}(\lambda)=1$. We analyze the $d_{k j}^{+}(\lambda)$ similarly, arriving at $d_{11}^{+}(\lambda)=\mathbf{O}\left(\left|\lambda^{-1 / 2} \ln \lambda\right|\right), \quad d_{12}^{+}(\lambda)=\mathbf{O}\left(\left|\lambda^{-1 / 2} \ln \lambda\right|\right), \quad d_{21}^{+}(\lambda)=\mathbf{O}_{a}\left(\left|\lambda^{-1}\right|\right)+$ $\mathbf{O}\left(\left|\lambda^{1 / 2} \ln \lambda\right|\right)$, and $d_{22}^{+}(\lambda)=\lambda^{-3 / 2}+m(\lambda)$, where $m(\lambda)=\mathbf{O}\left(\left|\lambda^{-1} \ln \lambda\right|\right)$. (This last decomposition is as in the case of Lemma 2.6.) 
Combining these observations and expanding $G_{\lambda}(x ; y)$ in detail, we have (for $0 \leq y \leq x)$

$$
\begin{aligned}
\left(\begin{array}{cc}
G_{\lambda} & G_{\lambda_{y}} \\
G_{\lambda_{x}} & G_{\lambda_{x y}}
\end{array}\right)= & \phi_{1}^{+}(x ; \lambda) d_{11}^{+}(\lambda) \tilde{\psi}_{1}^{+}(y ; \lambda)+\phi_{1}^{+}(x ; \lambda) d_{12}^{+}(\lambda) \tilde{\psi}_{2}^{+}(y ; \lambda) \\
& +\phi_{2}^{+}(x ; \lambda) d_{21}^{+}(\lambda) \tilde{\psi}_{1}^{+}(y ; \lambda)+\phi_{2}^{+}(x ; \lambda) d_{22}^{+}(\lambda) \tilde{\psi}_{2}^{+}(y ; \lambda) \\
& +\phi_{1}^{+}(x ; \lambda) \tilde{\phi}_{1}^{+}(y ; \lambda)+\lambda^{-3 / 2} \phi_{2}^{+}(x ; \lambda) \tilde{\phi}_{2}^{+}(y ; \lambda)
\end{aligned}
$$

Computing directly from Lemma 2.2 and from Lemma 2.6, we have

$$
\begin{aligned}
\Phi_{1}^{+}(x ; \lambda) d_{11}^{+}(\lambda) \widetilde{\Psi}_{1}^{+}(y ; \lambda)= & \left(\mathbf{O}\left(\left|\lambda^{-1 / 2} \ln \lambda\right|\right)\right) e^{\int_{K}^{x} \mu_{1}(s ; \lambda) d s-\int_{K}^{y} \mu_{3}(s ; \lambda) d s}, \\
\Phi_{1}^{+}(x ; \lambda) d_{11}^{+}(\lambda) \widetilde{\Psi}_{1}^{+\prime}(y ; \lambda)= & \mathbf{O}\left(\left|\lambda^{1 / 2} \ln \lambda\right|\right) e^{\int_{K}^{x} \mu_{1}(s ; \lambda) d s-\int_{K}^{y} \mu_{3}(s ; \lambda) d s}, \\
\Phi_{1}^{+}(x ; \lambda) d_{12}^{+}(\lambda) \widetilde{\Psi}_{2}^{+}(y ; \lambda)= & \left(\mathbf{O}\left(\left|\lambda^{-1 / 2} \ln \lambda\right|\right)+\mathbf{O}(|y|) \mathbf{O}(|\ln \lambda|)\right) e^{\int_{K}^{x} \mu_{1}(s ; \lambda) d s-\sqrt{\lambda y}}, \\
\Phi_{1}^{+}(x ; \lambda) d_{12}^{+}(\lambda) \widetilde{\Psi}_{2}^{+\prime}(y ; \lambda)= & \mathbf{O}\left(\left|\lambda^{1 / 2} \ln \lambda\right|\right) \mathbf{O}(|y|) e^{\int_{K}^{x} \mu_{1}(s ; \lambda) d s-\sqrt{\lambda y}}, \\
\Phi_{1}^{+}(x ; \lambda) \widetilde{\Phi}_{1}^{+}(y ; \lambda)= & \mathbf{O}_{a}(1) e^{\int_{y}^{x} \mu_{1}(s ; \lambda) d s}+\mathbf{O}\left(\left|\lambda^{1 / 2} \ln \lambda\right|\right) e^{\int_{y}^{x} \mu_{1}(s ; \lambda) d s}, \\
\Phi_{1}^{+}(x ; \lambda) \widetilde{\Phi}_{1}^{+\prime}(y ; \lambda)= & \mathbf{O}_{a}(1) e^{\int_{y}^{x} \mu_{1}(s ; \lambda) d s}+\mathbf{O}\left(\left|\lambda^{1 / 2} \ln \lambda\right|\right) e^{\int_{y}^{x} \mu_{1}(s ; \lambda) d s}, \\
\Phi_{2}^{+}(x ; \lambda) d_{21}^{+}(\lambda) \widetilde{\Psi}_{1}^{+}(y ; \lambda)= & \left(\mathbf{O}_{a}\left(\left|\lambda^{-1}\right|\right) \mathbf{O}\left(|x|^{-2}\right)+\mathbf{O}\left(\left|\lambda^{-1 / 2}\right|\right) \mathbf{O}\left(|x|^{-1}\right)+\mathbf{O}\left(|x|^{-2}\right)\right. \\
& \left.\times \mathbf{O}\left(\left|\lambda^{-1 / 2} \ln \lambda\right|\right)\right) e^{-\sqrt{\lambda} x-\int_{K}^{y} \mu_{3}(s ; \lambda) d s}, \\
\Phi_{2}^{+}(x ; \lambda) d_{21}^{+}(\lambda) \widetilde{\Psi}_{1}^{+\prime}(y ; \lambda)= & \left(\mathbf{O}\left(|x|^{-2}\right)+\mathbf{O}\left(|x|^{-2}\left|\lambda^{1 / 2} \ln \lambda\right|\right)+\mathbf{O}\left(\left|\lambda^{1 / 2}\right|\right) \mathbf{O}\left(|x|^{-1}\right)\right) \\
& \times e^{-\sqrt{\lambda} x-\int_{K}^{y} \mu_{3}(s ; \lambda) d s},
\end{aligned}
$$

and finally, according to Lemma 2.6,

$$
\begin{aligned}
& \Phi_{2}^{+}(x ; \lambda)\left(d_{22}^{+}(\lambda) \widetilde{\Psi}_{2}^{+}(y ; \lambda)+\lambda^{-3 / 2} \widetilde{\Phi}_{2}^{+}(y ; \lambda)\right) \\
& =e^{-\sqrt{\lambda}|x-y|}\left[\mathbf{O}\left(\left|\lambda^{-1 / 2}\right|\right) \mathbf{O}\left(|x|^{-1}\right) \mathbf{O}(|y|)+\frac{P_{1}^{+} \bar{u}_{x}}{\lambda}+\mathbf{O}\left(\left|\lambda^{-1 / 2}(\ln \lambda)^{2}\right|\right) \mathbf{O}\left(|x|^{-2}\right) \mathbf{O}(|y|)\right] \\
& \Phi_{2}^{+}(x ; \lambda)\left(d_{22}^{+}(\lambda) \widetilde{\Psi}_{2}^{+\prime}(y ; \lambda)+\lambda^{-3 / 2} \widetilde{\Phi}_{2}^{+\prime}(y ; \lambda)\right) \\
& =e^{-\sqrt{\lambda}|x-y|}\left[\mathbf{O}\left(|x|^{-2}\right) \mathbf{O}(|y|)+\mathbf{O}\left(\left|\lambda^{1 / 2} \ln \lambda\right|\right) \mathbf{O}\left(|x|^{-1}\right)+\mathbf{O}\left(\left|\lambda^{1 / 2}\right|\right) \mathbf{O}\left(|x|^{-1}\right) \mathbf{O}(|y|)\right. \\
& \left.+\mathbf{O}(|\ln \lambda|) \mathbf{O}\left(|x|^{-2}\right)\right] .
\end{aligned}
$$

Case (vi) $0<K \leq x \leq y$. The case $0<K \leq x \leq y$ is similar to Case (v), with only two new terms, namely,

$$
\begin{aligned}
\Psi_{1}^{+}(x ; \lambda) \widetilde{\Psi}_{1}^{+}(y ; \lambda) & =\mathbf{O}(1) e^{-\int_{x}^{y} \mu_{3}(s ; \lambda) d s}+\mathbf{O}\left(\left|\lambda^{1 / 2} \ln \lambda\right|\right) e^{-\int_{x}^{y} \mu_{3}(s ; \lambda) d s}, \\
\Psi_{1}^{+}(x ; \lambda) \widetilde{\Psi}_{1}^{+\prime}(y ; \lambda) & =\mathbf{O}_{a}(|\lambda|) e^{-\int_{x}^{y} \mu_{3}(s ; \lambda) d s}+\mathbf{O}\left(\left|\lambda^{1 / 2} \ln \lambda\right|\right) e^{-\int_{x}^{y} \mu_{3}(s ; \lambda) d s},
\end{aligned}
$$


and, according to Lemma 2.6,

$$
\begin{aligned}
& \left(\Phi_{2}^{+}(x ; \lambda) d_{22}(\lambda)-\lambda^{-3 / 2} \Psi_{2}^{+}(x ; \lambda)\right) \widetilde{\Psi}_{2}^{+}(y ; \lambda) \\
& =e^{-\sqrt{\lambda}|x-y|}\left[\mathbf{O}\left(\left|\lambda^{-1 / 2}\right|\right) \mathbf{O}\left(|x|^{-1}\right) \mathbf{O}(|y|)\right. \\
& \left.\quad+\mathbf{O}_{a}\left(\left|\lambda^{-1}\right|\right) \mathbf{O}\left(|x|^{-2}\right)+\mathbf{O}\left(\left|\lambda^{-1 / 2}(\ln \lambda)^{2}\right|\right) \mathbf{O}\left(|x|^{-1}\right)\right] \\
& \left(\Phi_{2}^{+}(x ; \lambda) d_{22}(\lambda)-\lambda^{-3 / 2} \Psi_{2}^{+}(x ; \lambda)\right) \widetilde{\Psi}_{2}^{+^{\prime}}(y ; \lambda)=e^{-\sqrt{\lambda}|x-y|} \mathbf{O}\left(|x|^{-1}\right) \mathbf{O}(|y|)
\end{aligned}
$$

Lemma 3.2 (Large $|\lambda| G_{\lambda}(x, y)$ estimates). For $\lambda$ bounded to the right of $\Gamma_{d}$ and for $|\lambda| \geq M$, some constant $M$ sufficiently large, there exist constants $C, \beta>0$ so that

$$
\begin{aligned}
\left|G_{\lambda}(x, y)\right| & \leq C\left|\lambda^{-1 / 2}\right| e^{-\beta^{-1 / 2}\left|\lambda^{1 / 2}\right||x-y|}, \\
\left|\partial_{y} G_{\lambda}(x, y)\right| & \leq C e^{-\beta^{-1 / 2}\left|\lambda^{1 / 2}\right||x-y|} .
\end{aligned}
$$

Proof. The large $|\lambda|$ behavior of $G_{\lambda}(x, y)$ depends on viscosity rather than convection and can be developed exactly as in the nondegenerate case. See in particular Zumbrun and Howard (1998/2002), p. 806.

Lemma 3.3 (Medium $|\lambda| G_{\lambda}(x, y)$ estimates). For $\lambda$ bounded to the right of $\Gamma_{d}$ and for $r \leq|\lambda| \leq R$, any fixed constants $R>r>0$, there exists $C>0$ so that

$$
\begin{aligned}
\left|G_{\lambda}(x, y)\right| & \leq C, \\
\left|\partial_{y} G_{\lambda}(x, y)\right| & \leq C .
\end{aligned}
$$

Proof. The medium $|\lambda|$ estimates follow from decay properties rather than decay rates and can be developed exactly as in the nondegenerate case. See in particular Zumbrun and Howard (1998/2002), p. 805.

\section{Estimates on $G(t, x ; y)$}

We now employ the estimates of Lemmas 3.1, 3.2, and 3.3 to derive estimates on the Green's function $G(t, x ; y)$ through the inverse Laplace transform representation

$$
G(t, x ; y)=\frac{1}{2 \pi i} \int_{\Gamma} e^{\lambda t} G_{\lambda}(x, y) d \lambda,
$$

where the contour of integration $\Gamma$ must encircle the poles of $G_{\lambda}$ (which occur at point spectrum for the operator $L$ ).

Before beginning the detailed proof of Theorem 1.1, we give a brief overview of the approach taken and set some notation. The analysis is divided into two cases, $|x-y| \leq \bar{K} t$ and $|x-y| \geq \bar{K} t$, where $\bar{K}$ is a constant chosen sufficiently large in the analysis. Generally speaking, in the case $|x-y| \leq \bar{K} t$, we use the estimates of Lemma 3.1 , while in the case $|x-y| \geq \bar{K} t$, we use the estimates of Lemma 3.2, though in all cases contours must eventually involve large $|\lambda|$ estimates.

In the cases for which we use Lemma 3.1, the estimates on $G_{\lambda}(x, y)$ are divided into a number of terms that can be integrated separately against $e^{\lambda t}$. For every such term, the contour of integration, $\Gamma$, will be chosen to depend on $t, x$, and $y$. In the 


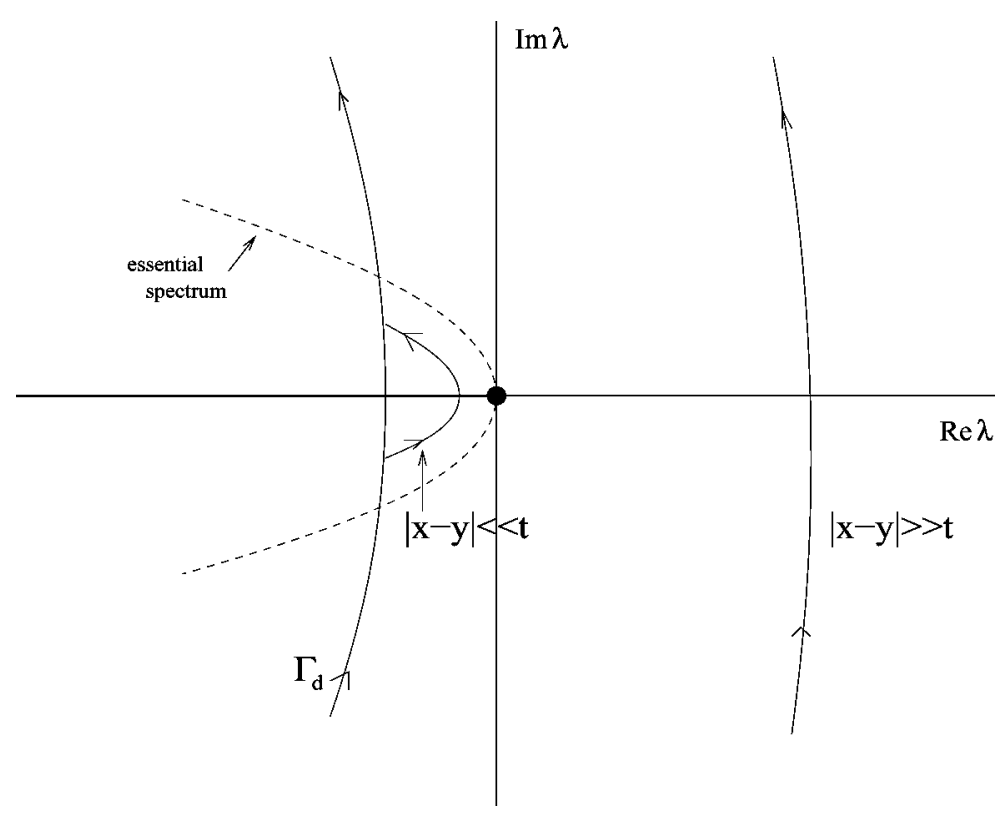

Figure 1. Contours in the case of analyticity at $\lambda=0$.

event that $|x-y| \gg t$, it will typically be advantageous to take a (parabolic) contour that crosses the real axis far to the right of the imaginary axis, while for $|x-y| \ll t$, it will typically be advantageous to take a contour to the left of the imaginary axis (see Figure 1). In either case, we only follow our contour of choice until it strikes the contour $\Gamma_{d}$, defined in (1.8), which aside from the point $\lambda=0$ lies to the right of the point spectrum of $L$. Throughout the analysis, then, for chosen contour $\Gamma$, we will use the notation $\Gamma^{*}$ to indicate the truncated portion of the contour that stops at $\Gamma_{d}$.

While there are a great many terms in $G_{\lambda}(x, y)$ to analyze, the analysis of several is similar. Two particularly important terms are the scattering and excited terms from the case $0 \leq x \leq y(|x-y| \leq \bar{K} t)$,

$$
\begin{aligned}
& S_{\lambda}(x, y)=\mathbf{O}\left(\left|\lambda^{-1 / 2}\right|\right) \mathbf{O}\left(|x|^{-1}\right) \mathbf{O}(|y|) e^{-\sqrt{\lambda}|x-y|}, \quad \text { and } \\
& E_{\lambda}(x, y)=\bar{u}_{x}(x) \mathbf{O}_{a}\left(\left|\lambda^{-1}\right|\right) e^{-\sqrt{\lambda}|x-y|}
\end{aligned}
$$

Though several estimates will be more technical than these, the fundamental ideas are all contained here. For the first we take the heat-equation-like contour defined through

$$
\sqrt{\lambda(k)}=\frac{|x-y|}{L t}+i k
$$

for which

$$
\lambda(k)=\frac{|x-y|^{2}}{L^{2} t^{2}}+2 i k \frac{|x-y|}{L t}-k^{2} ; \quad d \lambda=\left(2 i \frac{|x-y|}{L t}-2 k\right) d k,
$$




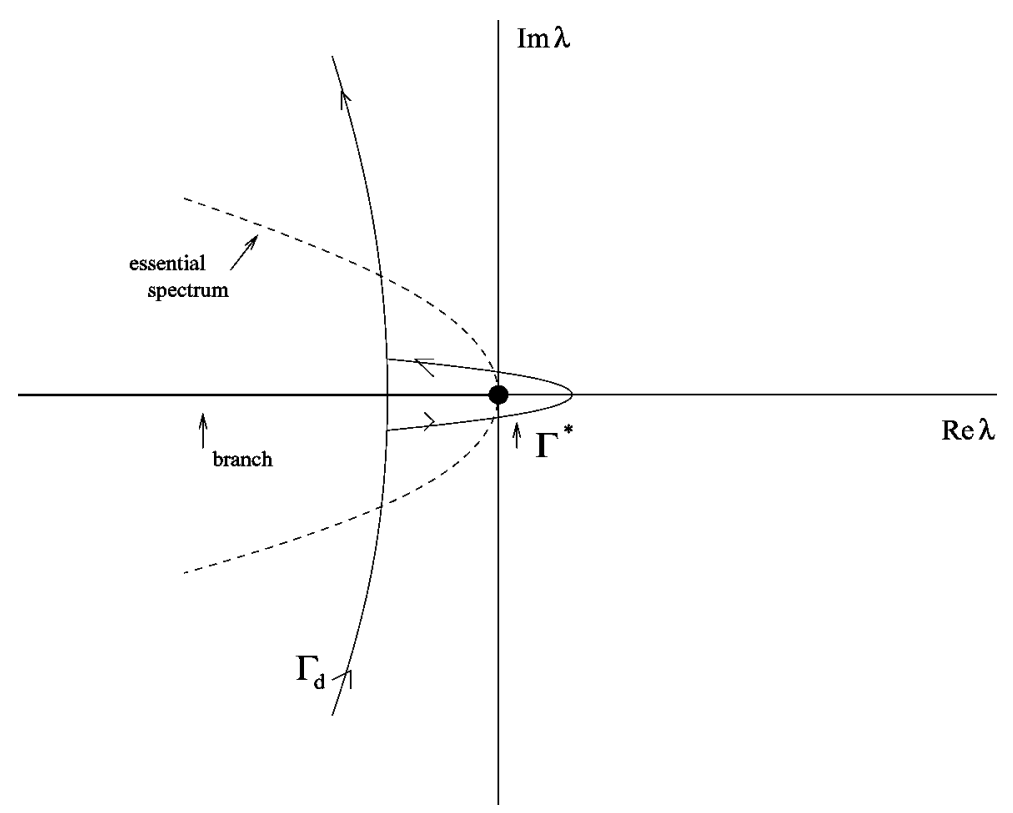

Figure 2. Contours in the case of a branch along $\operatorname{Re} \lambda<0$.

where $L$ is chosen large enough so that for $k$ sufficiently small, this contour remains in a small neighborhood of the origin. We follow this contour until it strikes $\Gamma_{d}$, and then follow $\Gamma_{d}$ out to the point at $\infty$ (see Figure 2).

Observe in particular that though our contour of choice in this case always crosses the real axis to the right of the imaginary axis, it moves rapidly into essential spectrum. Letting $\pm k^{*}$ represent the values of $k$ for which we strike $\Gamma_{d}$, we have

$$
\begin{aligned}
\left|\int_{\Gamma^{*}} e^{\lambda t} S_{\lambda}(x, y) d \lambda\right| & =\mathbf{O}\left(|x|^{-1}\right) \mathbf{O}(|y|) \int_{-k^{*}}^{+k^{*}} e^{\lambda(k) t} \mathbf{O}\left(|\lambda(k)|^{-1 / 2}\right) e^{-\sqrt{\lambda(k)}|x-y|} d \lambda \\
& =\mathbf{O}\left(|x|^{-1}\right) \mathbf{O}(|y|) \int_{-k^{*}}^{+k^{*}} e^{\frac{|x-y|^{2}}{L^{2} t^{2}} t-k^{2} t-\frac{|x-y|^{2}}{L t}}\left|\frac{2 i \frac{|x-y|}{L t}-2 k}{\frac{|x-y|}{L t}+i k}\right| d k \\
& =\mathbf{O}\left(t^{-1 / 2}\right) \mathbf{O}\left(|x|^{-1}\right) \mathbf{O}(|y|) e^{-\frac{(x-y)^{2}}{M t}} .
\end{aligned}
$$

Finally, along $\Gamma_{d}$, we observe that $\operatorname{Re} \sqrt{\lambda} \geq 0$ and $\mathbf{O}\left(\left|\lambda(k)^{-1 / 2}\right|\right)=\mathbf{O}(1)$, so that our integral decays at exponential rate in time, $e^{-\eta t}, \eta>0$. Since $|x-y| \leq \bar{K} t$, we have

$$
e^{-\eta t} \leq e^{-\eta t \frac{|x-y|^{2}}{K^{2} t^{2}}}=e^{-\frac{\eta}{K^{2}} \frac{(x-y)^{2}}{t}}
$$

which leads to our final estimate on this term

$$
\mathbf{O}\left(t^{-1 / 2}\right) \mathbf{O}\left(|x|^{-1}\right) \mathbf{O}(|y|) e^{-\frac{(x-y)^{2}}{M t}}
$$


For $E_{\lambda}(x, y)$, we again begin along the contour defined through $\sqrt{\lambda(k)}=\frac{|x-y|}{L t}+i k$, along which we have

$$
\begin{aligned}
\left|\bar{u}_{x}(x) \int_{\Gamma^{*}} \mathbf{O}_{a}\left(\left|\lambda^{-1}\right|\right) e^{\lambda t-\sqrt{\lambda}|x-y|} d \lambda\right| & =\bar{u}_{x}(x) \int_{-k^{*}}^{+k^{*}} e^{-\frac{(x-y)^{2}}{M t}-k^{2} t} \frac{\left|2 i \frac{|x-y|}{L t}-2 k\right|}{\left|\frac{|x-y|^{2}}{L^{2} t^{2}}+i k \frac{|x-y|}{t}-k^{2}\right|}|d k| \\
& =\mathbf{O}\left(|x|^{-2}\right) \mathbf{O}\left(\frac{t}{|x-y|}\right) \mathbf{O}\left(t^{-1 / 2}\right) e^{-\frac{(x-y)^{2}}{M t}}
\end{aligned}
$$

In the event that $|x-y| \geq \epsilon_{0} \sqrt{t}$, we observe that $\frac{t^{1 / 2}}{|x-y|} \leq \frac{1}{\epsilon_{0}}$, so that we have an estimate by (recalling that we are considering the case $0 \leq x \leq y$ )

$$
\mathbf{O}\left(t^{-1 / 2}\right) \mathbf{O}\left(|x|^{-2}\right) \mathbf{O}(|x-y|) e^{-\frac{(x-y)^{2}}{M t}}=\mathbf{O}\left(t^{-1 / 2}\right) \mathbf{O}\left(|x|^{-2}\right) \mathbf{O}(|y|) e^{-\frac{(x-y)^{2}}{M t}} .
$$

In the event that $|x-y| \leq \epsilon_{0} \sqrt{t}$, we proceed as in Howard (2002a,b) and divide the integrand into an analytic term and an error, as

$$
\begin{aligned}
& \frac{1}{2 \pi i} \int_{\Gamma^{*}} \frac{P_{+} \bar{u}_{x}(x) e^{\lambda t-\sqrt{\lambda}|x-y|}}{\lambda} d \lambda \\
& \quad=\frac{1}{2 \pi i} \int_{\Gamma^{*}} \frac{P_{+} \bar{u}_{x}(x) e^{\lambda t}}{\lambda} d \lambda+\frac{1}{2 \pi i} \int_{\Gamma^{*}} \frac{P_{+} \bar{u}_{x}(x) e^{\lambda t}\left(e^{-\sqrt{\lambda}|x-y|}-1\right)}{\lambda} d \lambda .
\end{aligned}
$$

Here, we have observed that for $\mathbf{O}_{a}\left(\left|\lambda^{-1}\right|\right)$ meromorphic in $\lambda$ in a neighborhood of the origin, we have

$$
\mathbf{O}_{a}\left(\left|\lambda^{-1}\right|\right)=\frac{P_{+}}{\lambda}+\mathbf{O}_{a}(1)
$$

whence

$$
E_{\lambda}(x, y)=\bar{u}_{x}(x) \mathbf{O}_{a}\left(\left|\lambda^{-1}\right|\right) e^{-\sqrt{\lambda}|x-y|}=\frac{P_{+} \bar{u}_{x}(x)}{\lambda} e^{-\sqrt{\lambda}|x-y|}+\mathbf{O}\left(|x|^{-2}\right) e^{-\sqrt{\lambda}|x-y|},
$$

the second of which can be subsumed into $S_{\lambda}(x, y)$. For the first integral on the righthand side of Equation (4.1), we employ analyticity of numerator and denominator to proceed similarly as in the nondegenerate analysis of Zumbrun and Howard (1998/2002) and shift our contour to the left of the imaginary axis, using Cauchy's integral formula to compute the residue. Our estimate on this term becomes

$$
P_{+} \bar{u}_{x}(x) I_{\left\{|x-y| \leq \epsilon_{0} \sqrt{t}\right\}}
$$

For the second integral, we first consider the strip of $\Gamma^{*}$ over which $|\sqrt{\lambda}(x-y)| \leq 1$, for which we have

$$
\begin{aligned}
\left|\int_{\Gamma^{*}} \frac{\bar{u}_{x}(x) e^{\lambda t}\left(e^{-\sqrt{\lambda}|x-y|}-1\right)}{\lambda} d \lambda\right| & =\mathbf{O}\left(|x|^{-2}\right)\left|\int_{\Gamma^{*}} \frac{e^{\lambda t} \mathbf{O}(|\sqrt{\lambda}(x-y)|)}{\lambda} d \lambda\right| \\
& =\mathbf{O}\left(|x|^{-2}\right) \mathbf{O}(|y|) \int_{\Gamma^{*}} \mathbf{O}\left(\left|\lambda^{-1 / 2}\right|\right) e^{\lambda t} d \lambda \\
& =\mathbf{O}\left(|x|^{-2}\right) \mathbf{O}(|y|) \mathbf{O}\left(t^{-1 / 2}\right) e^{-\frac{(x-y)^{2}}{L t}},
\end{aligned}
$$


where we have made use of the observation that for $|x-y| \leq \epsilon_{0} \sqrt{t}, \quad 1 \leq$ $C e^{-\frac{(x-y)^{2}}{L t}}$. On the other hand, for $|\sqrt{\lambda}(x-y)|>1$, we have $\left|\lambda^{-1 / 2}\right|=\mathbf{O}(|x-y|)$ and consequently the estimate

$$
\left|\int_{\Gamma^{*}} \frac{\bar{u}_{x}(x) e^{\lambda t}\left(e^{-\sqrt{\lambda}|x-y|}-1\right)}{\lambda} d \lambda\right|=\mathbf{O}\left(|x|^{-2}\right)\left|\int_{\Gamma^{*}} e^{\lambda t} \mathbf{O}\left(\left|\lambda^{-1 / 2}\right|\right) \mathbf{O}(|x-y|) d \lambda\right|,
$$

as above. Our final estimate becomes

$$
\mathbf{O}\left(t^{-1 / 2}\right) \mathbf{O}\left(|x|^{-2}\right) \mathbf{O}(|y|) e^{-\frac{(x-y)^{2}}{M t}}+P_{+} \bar{u}_{x}(x) I_{\left\{|x-y| \leq \epsilon_{0} \sqrt{t}\right\}} .
$$

Note in particular that in this analysis we have determined critical tracking information regarding how rapidly mass in the far field contributes to a shift of the shock wave. According to our choice of shift $\delta(t)$ in Equation (1.4), we will have a linear term of the form

$$
\delta(t) \sim P_{+} \int_{0}^{\epsilon_{0} \sqrt{t}} v_{0}(y) d y,
$$

for which we observe that the window of mass that has accumulated in the shock layer at time $t$ is $\left[0, \epsilon_{0} \sqrt{t}\right]$.

Proof of Theorem 1.1. In the small time case $|x-y| \geq \bar{K} t$, some $\bar{K}$ sufficiently large, we can proceed via the large $|\lambda|$ estimates of Lemma 3.2, which are exactly the same estimates as in Zumbrun and Howard (1998/2002). In this case, we take the contour defined through

$$
\sqrt{\lambda(k)}=\frac{|x-y|}{2 \sqrt{\beta} t}+i k
$$

from which an analysis precisely as that of the heat kernel yields estimates of the form

$$
|G(t, x ; y)| \leq C t^{-1 / 2} e^{-\frac{(x-y)^{2}}{4 \beta t}}
$$

which can be subsumed into the estimates of Theorem 1.1.

For the case $|x-y| \leq \bar{K} t$, we proceed in a number of subcases.

Case (i) $y \leq x \leq 0$. According to Lemma 3.1, we have five integrals to evaluate in the case $y \leq x \leq 0$, beginning with the integrands $\mathbf{O}_{a}(1) e^{\mu_{2}^{-}(\lambda)(x-y)}$, $\mathbf{O}_{a}(1) e^{\mu_{3}^{-}(\lambda) x-\mu_{2}^{-}(\lambda) y}$, and $\bar{u}_{x}(x) \overline{\mathbf{O}}_{a}\left(\left|\lambda^{-1}\right|\right) e^{-\mu_{2}^{-}(\lambda) y}$. Each of these arises in the case of nondegenerate waves and can be analyzed as in Zumbrun and Howard (1998/2002). Summarizing, we have

$$
\begin{gathered}
\int_{\Gamma} \mathbf{O}_{a}(1) e^{\lambda t+\mu_{2}^{-}(\lambda)(x-y)} d \lambda=\mathbf{O}\left(t^{-1 / 2}\right) e^{-\frac{\left(x-y-a_{2}^{-} t\right)^{2}}{M t}}, \\
\int_{\Gamma} \mathbf{O}_{a}(1) e^{\lambda t+\mu_{3}^{-}(\lambda) x-\mu_{2}^{-}(\lambda) y} d \lambda=\mathbf{O}\left(t^{-1 / 2}\right) e^{-\frac{\left(x-\frac{a_{1}^{-}}{a_{2}} y-a_{1}^{-} t\right.}{M t}}, \\
\bar{u}_{x}(x) \int_{\Gamma} \mathbf{O}_{a}\left(\left|\lambda^{-1}\right|\right) e^{\lambda t-\mu_{2}^{-}(\lambda) y} d \lambda=\bar{u}_{x}(x)\left(\mathbf{O}(1) e^{-\frac{\left(y+a_{2}^{-} t\right)^{2}}{M t}}+\mathbf{O}(1) I_{\left\{|y| \leq\left|a_{2}^{-}\right| t\right\}}\right) .
\end{gathered}
$$


For the term $\mathbf{O}\left(\left|\lambda^{1 / 2} \ln \lambda\right|\right) e^{\mu_{3}^{-}(\lambda) x-\mu_{2}^{-}(\lambda) y}$, we must alter the analysis employed above so as to avoid $\lambda \in \mathbb{R}_{-}$, where we lose analyticity. Expanding $\mu_{2}^{-}(\lambda)$ and $\mu_{3}^{-}(\lambda)$, we have

$$
\begin{aligned}
& \mu_{2}^{-}(\lambda)=\frac{a_{2}^{-}-\sqrt{\left(a_{2}^{-}\right)^{2}+4 \lambda}}{2}=-\frac{1}{a_{2}^{-}} \lambda+\frac{1}{\left(a_{2}^{-}\right)^{3}} \lambda^{2}+\mathbf{O}\left(\lambda^{3}\right), \quad\left(\text { Recall }: a_{2}^{-}>0\right) \\
& \mu_{3}^{-}(\lambda)=\frac{a_{1}^{-}+\sqrt{\left(a_{1}^{-}\right)^{2}+4 \lambda}}{2}=-\frac{1}{a_{1}^{-}} \lambda+\frac{1}{\left(a_{1}^{-}\right)^{3}} \lambda^{2}+\mathbf{O}\left(\lambda^{3}\right), \quad\left(\text { Recall }: a_{1}^{-}<0\right) .
\end{aligned}
$$

Our principal contour will be chosen as in Zumbrun and Howard (1998/2002) by the relation

$$
\begin{aligned}
& \left(-\frac{x}{a_{1}^{-}}+\frac{y}{a_{2}^{-}}\right) \lambda(k)+\left(\frac{x}{\left(a_{1}^{-}\right)^{3}}-\frac{y}{\left(a_{2}^{-}\right)^{3}}\right) \lambda(k)^{2} \\
& =\left(-\frac{x}{a_{1}^{-}}+\frac{y}{a_{2}^{-}}\right) \lambda_{R}+\left(\frac{x}{\left(a_{1}^{-}\right)^{3}}-\frac{y}{\left(a_{2}^{-}\right)^{3}}\right) \lambda_{R}^{2}+i\left(-\frac{x}{a_{1}^{-}}+\frac{y}{a_{2}^{-}}\right) k,
\end{aligned}
$$

where $\lambda_{R}$ is the critical point where the contour crosses the real axis. Expanding $\lambda(k)=\lambda_{R}+\lambda_{1} k+\lambda_{2} k^{2}+\cdots$, we immediately find $\lambda_{1}=i\left(1+\mathbf{O}\left(\lambda_{R}\right)\right)$ and

$$
\lambda_{2}=\frac{\frac{x}{\left(a_{1}^{-}\right)^{3}}-\frac{y}{\left(a_{2}^{-}\right)^{3}}}{-\frac{x}{a_{1}^{-}}+\frac{y}{a_{2}^{-}}}\left(1+\mathbf{O}\left(\lambda_{R}\right)\right)
$$

for which $\lambda_{2} \leq-\gamma<0$, some fixed $\gamma$. We choose $\lambda_{R}$ optimally by minimizing the exponent function

$$
g\left(\lambda_{R}\right)=\lambda_{R} t+\left(-\frac{x}{a_{1}^{-}}+\frac{y}{a_{2}^{-}}\right) \lambda_{R}+\left(\frac{x}{\left(a_{1}^{-}\right)^{3}}-\frac{y}{\left(a_{2}^{-}\right)^{3}}\right) \lambda_{R}^{2},
$$

for

$$
\lambda_{R}=\frac{\bar{\alpha}_{-}}{\bar{p}_{-}} ; \quad \bar{\alpha}_{-}:=\frac{x-\frac{a_{1}^{-}}{a_{2}^{-}} y-a_{1}^{-} t}{2 t} ; \quad \bar{p}_{-}:=\left(\frac{x}{\left(a_{1}^{-}\right)^{2} t}-\frac{a_{1}^{-} y}{\left(a_{2}^{-}\right)^{3} t}\right) \leq 0 .
$$

Note in particular that our contour of choice is entirely determined by our choice of $\lambda_{R}$. Following Zumbrun and Howard (1998/2002) in the case $\frac{\bar{\alpha}_{-}}{\bar{p}_{-}} \geq 0$ (that is, for $\left.\left|x-\frac{a_{1}^{-}}{a_{2}^{-}} y\right| \geq\left|a_{1}^{-}\right| t\right)$, we take

$$
\lambda_{R}=\left\{\begin{array}{lc}
+\epsilon, & \epsilon \leq \frac{\bar{\alpha}_{-}}{\bar{p}_{-}} \leq M, \\
\frac{\bar{\alpha}_{-},}{\bar{p}_{-}} & t^{-1 / 2} \leq \frac{\bar{\alpha}_{-}}{\bar{p}_{-}} \leq \epsilon, \\
t^{-1 / 2}, & 0 \leq \frac{\bar{\alpha}_{-}}{\bar{p}_{-}} \leq t^{-1 / 2}
\end{array}\right.
$$

to obtain an estimate by

$$
\mathbf{O}\left(t^{-3 / 4} \ln t\right) e^{-\frac{\left(x-\frac{a_{1}^{-}}{a_{2}} y-a_{1}^{-} t\right.}{M t}},
$$


which can be subsumed into estimates (4.2). The difficulty arises in the case $\frac{\bar{\alpha}_{-}}{\bar{p}_{-}}<0$ (that is, $\left|x-\frac{a_{1}^{-}}{a_{2}^{-}} y\right| \leq\left|a_{1}^{-}\right| t$ ), when the nondegenerate analysis would select $\lambda_{R}<0$, according to

$$
\lambda_{R}= \begin{cases}-\epsilon, & -\epsilon \geq \frac{\bar{\alpha}_{-}}{\bar{p}_{-}} \\ \bar{\alpha}_{-}, & -t^{-1 / 2} \geq \frac{\bar{\alpha}_{-}}{\bar{p}_{-}} \geq-\epsilon \\ -t^{-1 / 2}, & 0 \geq \frac{\bar{\alpha}_{-}}{\bar{p}_{-}} \geq-t^{-1 / 2},\end{cases}
$$

which is precluded by our branch along $\lambda \in \mathbb{R}_{-}$. At this point, we arrive at the primary new feature of the degenerate-wave contour analysis. The idea in the nondegenerate case was, for $t \gg|x-y|$, to take contours that remain entirely in the negative real half-plane and thus take advantage of exponential time decay, which dominates the exponential growth in $|x|$ and $|y|$. In the degenerate case, we cannot avoid passing our contours through the positive real axis, and our new approach will be to move our contours quickly into the negative real half-plane.

We first observe that we can integrate along essential spectrum

$$
\Gamma_{\text {ess }}=\left\{\lambda: \text { Either } \operatorname{Re} \mu_{2}^{-}(\lambda)=0 \text { or } \operatorname{Re} \mu_{3}^{-}(\lambda)=0\right\}
$$

to obtain an estimate

$$
\mathbf{O}\left(t^{-3 / 4} \ln t\right) I_{\left\{\left|x-\frac{a_{1}^{-}}{a_{2}^{-}} y\right| \leq\left|a_{1}^{-}\right| t\right\}} \cdot
$$

Alternatively, we can move more rapidly into essential spectrum by taking the heat-equation-like contour

$$
\sqrt{\lambda(k)}=t^{-1}+i k
$$

which we denote $\Gamma_{D}$, until it strikes the non-degenerate contour described above. Along $\Gamma_{D}$ we have

$$
\begin{aligned}
\lambda(k) & =t^{-2}+2 i k t^{-1}-k^{2} ; \quad d \lambda=\left(2 i t^{-1}-2 k\right) d k ; \quad \text { and } \\
\operatorname{Re} \lambda(k)^{2} & =t^{-4}+k^{4}-6 k^{2} t^{-2},
\end{aligned}
$$

so that

$$
\begin{aligned}
\left|\int_{\Gamma_{D}^{*}} \mathbf{O}\left(\left|\lambda^{1 / 2} \ln \lambda\right|\right) e^{\lambda t+\mu_{3}^{-}(\lambda) x-\mu_{2}^{-}(\lambda) y} d \lambda\right| \leq & C \int_{-k^{*}}^{+k^{*}}\left|2 i t^{-1}-2 k \|\left(t^{-1}+i k\right)\left[\ln \left(t^{-1}+i k\right)\right]\right| \\
& \times e^{-k^{2}\left(t-\frac{x}{a_{1}^{-}}+\frac{y}{a_{2}^{2}}\right)+k^{4}\left(\frac{x}{\left(a_{1}^{-}\right)^{3}}-\frac{y}{\left(a_{2}^{-}\right)^{3}}\right)} d k
\end{aligned}
$$

We observe here that for $|k|$ sufficiently large, we cannot get a good estimate along this contour. The index $k^{*}$, then, where we cross the nondegenerate contour, is 
critical. Indexing our nondegenerate contour by $l$, we have

$$
\lambda(l)=\lambda_{R}+i l\left(1+\mathbf{O}\left(\lambda_{R}\right)\right)+\frac{\left(\frac{x}{\left(a_{1}^{-}\right)^{3}}-\frac{y}{\left(a_{2}^{-}\right)^{3}}\right)}{\left(-\frac{x}{a_{1}^{-}}+\frac{y}{a_{2}^{-}}\right)} l^{2}\left(1+\mathbf{O}\left(\lambda_{R}\right)\right)+\mathbf{O}\left(l^{3}\right),
$$

for which the intersection with $\Gamma_{D}$ occurs for

$$
k^{2}=\frac{t^{-2}-\lambda_{R}}{1+\mathbf{O}\left(t^{-2}\right)},
$$

for which we recall that in this case $\lambda_{R}<0$. In the first case, $t^{-1 / 2} \leq \frac{\bar{\alpha}_{-}}{\bar{p}_{-}} \leq 0$, we choose $\lambda_{R}=-t^{-1 / 2}$, for which (for $t \geq 1$ ) we have $|k| \leq C t^{-1 / 4}$. In this case, since $\left|x-\frac{a_{1}^{-}}{a_{2}^{-}} y\right| \leq\left|a_{1}^{-}\right| t$, the exponent $k^{4}\left(\frac{x}{\left(a_{1}^{-}\right)^{3}}-\frac{y}{\left(a_{2}^{-}\right)^{3}}\right)$ is bounded, and we can integrate over $|k| \leq C t^{-1 / 4}$ to obtain an estimate by

$$
\mathbf{O}\left(\left|x-\frac{a_{1}^{-}}{a_{2}^{-}} y-a_{1}^{-} t\right|^{-3 / 2} \ln t\right) I_{\left\{\left|x-\frac{a_{1}^{-}}{a_{2}^{2}} y\right| \leq\left|a_{1}^{-}\right| t\right\}} .
$$

In the case $-\epsilon \leq \frac{\bar{\alpha}_{-}}{\bar{p}_{-}} \leq-t^{-1 / 2}$, we choose

$$
\lambda_{R}=\frac{\bar{\alpha}_{-}}{\bar{p}_{-}}=-\frac{1}{2} \frac{\left(t-\frac{x}{a_{1}^{-}}+\frac{y}{a_{2}^{-}}\right)}{\frac{x}{\left(a_{1}^{-}\right)^{3}}-\frac{y}{\left(a_{2}^{-}\right)^{3}}}
$$

for which our degenerate contour intersects with our nondegenerate contour for

$$
k^{2} \leq \frac{1}{2} \frac{\left(t-\frac{x}{a_{1}^{-}}+\frac{y}{a_{2}^{-}}\right)}{\frac{x}{\left(a_{1}^{-}\right)^{3}}-\frac{y}{\left(a_{2}^{-}\right)^{3}}}+\mathbf{O}\left(t^{-2}\right) .
$$

For this range of $k$, we have

$$
\begin{aligned}
-k^{2}\left(t-\frac{x}{a_{1}^{-}}+\frac{y}{a_{2}^{-}}\right)+k^{4}\left(\frac{x}{\left(a_{1}^{-}\right)^{3}}-\frac{y}{\left(a_{2}^{-}\right)^{3}}\right) & =-\frac{k^{2}}{\frac{x}{\left(a_{1}^{-}\right)^{3}}-\frac{y}{\left(a_{2}^{-}\right)^{3}}}\left(\frac{t-\frac{x}{a_{1}^{-}}+\frac{y}{a_{2}^{-}}}{\frac{x}{\left(a_{1}^{-}\right)^{3}}-\frac{y}{\left(a_{2}^{-}\right)^{3}}}-k^{2}\right) \\
& \leq-\frac{1}{2} k^{2}\left(t-\frac{x}{a_{1}^{-}}+\frac{y}{a_{2}^{-}}\right)+\mathbf{O}\left(t^{-1}\right),
\end{aligned}
$$

for which we can integrate over $k$ as above. In the final case, $\frac{\bar{\alpha}_{-}}{\bar{p}_{-}} \leq-\epsilon$, we observe that

$$
\operatorname{Re}\left(-k^{2}\left(t-\frac{x}{a_{1}^{-}}+\frac{y}{a_{2}^{-}}\right)+k^{4}\left(\frac{x}{\left(a_{1}^{-}\right)^{3}}-\frac{y}{\left(a_{2}^{-}\right)^{3}}\right)\right)=-\frac{k^{2}}{\frac{x}{\left(a_{1}^{-}\right)^{3}}-\frac{y}{\left(a_{2}^{-}\right)^{3}}}\left(-2 \frac{\bar{\alpha}_{-}}{\bar{p}_{-}}-k^{2}\right),
$$

for which we have decay as in the previous cases for $k^{2} \leq \epsilon$. We can choose $d_{0}$ (for the contour $\Gamma_{d}$ defined in (1.8)) sufficiently small so that we strike $\Gamma_{d}$ for $k^{2} \leq \epsilon$. The nondegenerate analysis of Zumbrun and Howard (1998/2002) applies along $\Gamma_{d}$, and we obtain an estimate that can be subsumed into those above. 
The remaining term in this case, $\mathbf{O}\left(\left|\lambda^{-1 / 2} \ln \lambda\right|\right) e^{-\mu_{2}^{-}(\lambda) y}$, can be analyzed as in the case

$$
\mathbf{O}\left(\lambda^{1 / 2} \ln \lambda\right) e^{\mu_{3}^{-}(\lambda) x-\mu_{2}^{-}(\lambda) y},
$$

with $x$ set to zero. The estimates on $G_{y}(t, x ; y)$ follow similarly.

Case (ii) $x \leq y \leq 0$. According to Lemma 3.1, we have five integrands to evaluate in the case $x \leq y \leq 0$, though the only integrand not examined in the analysis of Case (i) is $\mathbf{O}_{a}(1) e^{\mu_{3}^{-}(\lambda)(x-y)}$, which arises in the nondegenerate case and has been analyzed in Zumbrun and Howard (1998/2002), with an estimate by

$$
\mathbf{O}\left(t^{-1 / 2}\right) e^{-\frac{\left(x-y-a_{1}^{-} t\right)^{2}}{M t}}
$$

Estimates on $G_{y}(t, x ; y)$ in this case follow similarly.

Case (iii) $x \leq 0<K \leq y$. According to Lemma 3.1, we have seven integrals to evaluate in the case $x \leq 0<K \leq y$, beginning with the integrand $\mathbf{O}_{a}(1) e^{\mu_{3}^{-}(\lambda) x-\int_{K}^{y} \mu_{3}(s ; \lambda) d s}$. Since $\mu_{3}(x ; \lambda)$ is analytic in $\lambda$ in a neighborhood of $\lambda=0$, this integral can be analyzed similarly as in the nondegenerate case. We must, however, keep track of the $y$-dependence in $\mu_{3}$ through the Taylor expansion

$$
\int_{K}^{y} \mu_{3}(s ; \lambda) d s=-\lambda \int_{K}^{y} \frac{d s}{a_{1}(s)}+\lambda^{2} \int_{K}^{y} \frac{d s}{a_{1}(s)^{3}}+\mathbf{O}\left(\lambda^{3}\right),
$$

for which we take $y \geq K$. (In the event $y \leq K$, we can subsume $y$ behavior into $\mathbf{O}_{a}(1)$ and proceed with an estimate for the case $y=0$.) Consequently, we choose our contour $\lambda(k)$ through

$$
\begin{aligned}
& \left(-\frac{x}{a_{1}^{-}}+\int_{K}^{y} \frac{d s}{a_{1}(s)}\right) \lambda(k)+\left(\frac{x}{\left(a_{1}^{-}\right)^{3}}-\int_{K}^{y} \frac{d s}{a_{1}(s)^{3}}\right) \lambda(k)^{2} \\
& \quad=\left(-\frac{x}{a_{1}^{-}}+\int_{K}^{y} \frac{d s}{a_{1}(s)}\right) \lambda_{R}+\left(\frac{x}{\left(a_{1}^{-}\right)^{3}}-\int_{K}^{y} \frac{d s}{a_{1}(s)^{3}}\right) \lambda_{R}^{2}+i k\left(-\frac{x}{a_{1}^{-}}+\int_{K}^{y} \frac{d s}{a_{1}(s)}\right),
\end{aligned}
$$

for which

$$
\lambda(k)=\lambda_{R}+i k\left(1+\mathbf{O}\left(\lambda_{R}\right)\right)+\gamma(x, y) k^{2}\left(1+\mathbf{O}\left(\lambda_{R}\right)\right)+\mathbf{O}\left(k^{3}\right)
$$

where

$$
\gamma(x, y)=\frac{\left(\frac{x}{\left(a_{1}^{-}\right)^{3}}-\int_{K}^{y} \frac{d s}{a_{1}(s)^{3}}\right)}{\left(-\frac{x}{a_{1}^{-}}+\int_{K}^{y} \frac{d s}{a_{1}(s)}\right)} \leq \gamma_{0}<0 .
$$

Similarly as in the analysis of Case (i), we choose our principal value of $\lambda_{R}$ to minimize

$$
g\left(\lambda_{R}\right)=\lambda_{R} t+\left(-\frac{x}{a_{1}^{-}}+\int_{K}^{y} \frac{d s}{a_{1}(s)}\right) \lambda_{R}+\left(\frac{x}{\left(a_{1}^{-}\right)^{3}}-\int_{K}^{y} \frac{d s}{a_{1}(s)^{3}}\right) \lambda_{R}^{2},
$$


so that

$$
\lambda_{R}=\frac{\tilde{\alpha}}{\tilde{p}} ; \quad \tilde{\alpha}:=\frac{x-a_{1}^{-} \int_{K}^{y} \frac{d s}{a_{1}(s)}-a_{1}^{-} t}{2 t} ; \quad \tilde{p}:=\frac{\left(\frac{x}{\left(a_{1}^{-}\right)^{2}}-a_{1}^{-} \int_{K}^{y} \frac{d s}{a_{1}(s)^{3}}\right)}{t} .
$$

We can now proceed as in Case (i) with

$$
\lambda_{R}=\left\{\begin{array}{lc} 
\pm \epsilon, & \pm \epsilon \lesseqgtr \frac{\tilde{\alpha}}{\tilde{p}} \lesseqgtr \pm M \\
\frac{\tilde{\alpha}}{\tilde{p}}, & t^{-1 / 2} \leq\left|\frac{\tilde{\alpha}}{\tilde{p}}\right| \leq \epsilon . \\
\pm t^{-1 / 2}, & 0 \lesseqgtr \frac{\tilde{\alpha}}{\tilde{p}} \lesseqgtr \pm t^{-1 / 2}
\end{array}\right.
$$

The final estimate becomes

$$
\mathbf{O}\left(t^{-1 / 2}\right) e^{-\frac{\left(x-a_{1}^{-} \int_{K}^{y} \frac{d s}{a_{1}(s)}-a_{1}^{-} t\right)^{2}}{M t}}
$$

For the integrand $\bar{u}_{x}(x) \mathbf{O}_{a}\left(\left|\lambda^{-1}\right|\right) e^{-\int_{K}^{y} \mu_{3}(s ; \lambda) d s}$, we can proceed again as in the case of nondegenerate waves, with $\mu_{3}(x ; \lambda)$ treated as above. We find

$$
\int_{\Gamma} \mathbf{O}_{a}\left(\left|\lambda^{-1}\right|\right) \bar{u}_{x}(x) e^{\lambda t-\int_{K}^{y} \mu_{3}(s ; \lambda) d s} d \lambda=\bar{u}_{x}(x)\left(\mathbf{O}(1) e^{-\frac{\left(\int_{K}^{y} \frac{d s}{a_{1}(s)}+a_{1}^{-} t\right)^{2}}{M t}}+\mathbf{O}(1) I_{\left\{\left|\int_{K}^{y} \frac{d s}{a_{1}(s)}\right| \leq t\right\}}\right) .
$$

For the integrands $\mathbf{O}\left(\left|\lambda^{1 / 2} \ln \lambda\right|\right) e^{\mu_{3}^{-}(\lambda) x-\int_{K}^{y} \mu_{3}(s ; \lambda) d s} \quad$ and $\quad \bar{u}_{x}(x) \mathbf{O}\left(\left|\lambda^{-1 / 2} \ln \lambda\right|\right)$ $e^{-\int_{K}^{y} \mu_{3}(s ; \lambda) d s}$, we proceed as in the analysis of $\mathbf{O}\left(\left|\lambda^{1 / 2} \ln \lambda\right|\right) e^{\mu_{3}^{-}(\lambda) x-\mu_{2}^{-}(\lambda) y}$ in Case (i). We obtain estimates that can be subsumed into those above, as well as the additional estimate

$$
\left[\mathbf{O}\left(t^{-3 / 4} \ln t\right) \wedge \mathbf{O}\left(\left|x-a_{1}^{-} \int_{K}^{y} \frac{d s}{a_{1}(s)}-a_{1}^{-} t\right|^{-3 / 2} \ln t\right)\right] I_{\left\{\left|x-a_{1}^{-} \int_{K}^{y} \frac{d s}{a_{1}(s)}\right| \leq\left|a_{1}^{-}\right| t\right\}},
$$

which we observe for $t$ bounded away from zero can be alternatively recorded in the useful form,

$$
\mathbf{O}\left(\left(1+\left|x-a_{1}^{-} \int_{K}^{y} \frac{d s}{a_{1}(s)}-a_{1}^{-} t\right|+t^{1 / 2}\right)^{-3 / 2} \ln (e+t)\right) I_{\left\{\left|x-a_{1}^{-} \int_{K}^{y} \frac{d s}{a_{1}(s)}\right| \leq\left|a_{1}^{-}\right| t\right\}} .
$$

The fundamentally new terms in this case are $\mathbf{O}(1) e^{\mu_{3}^{-}(\lambda) x-\sqrt{\lambda} y}$ and

$$
\bar{u}_{x}(x)\left(\mathbf{O}_{a}\left(\left|\lambda^{-1}\right|\right)+\mathbf{O}\left(\left|\lambda^{-1 / 2} \ln \lambda\right|\right)\right) e^{-\sqrt{\lambda} y} .
$$

In both cases, we first observe that for $\frac{\tilde{\alpha}}{\tilde{p}} \geq 0$, we may take advantage of the observation that along the contour chosen for the integrand $\mathbf{O}_{a}(1) e^{\mu_{3}^{-}(\lambda) x-\int_{K}^{y} \mu_{3}(s ; \lambda)}$, and for $|\lambda|$ sufficiently small, we have $\operatorname{Re} \sqrt{\lambda} y \geq \operatorname{Re} \int_{K}^{y} \mu_{3}(s ; \lambda) d s$, so that the estimates obtained can be subsumed into those above. For $\frac{\tilde{\alpha}}{\tilde{p}}<0$, we begin with the integrand $\mathbf{O}(1) e^{\mu_{3}^{-}(\lambda) x-\sqrt{\lambda} y}$, for which we proceed similarly as in the Case (i) analysis of $\mathbf{O}\left(\left|\lambda^{1 / 2} \ln \lambda\right|\right) e^{\mu_{3}^{-}(\lambda) x-\mu_{2}^{-}(\lambda) y}$, by taking the degenerate contour defined through 
$\sqrt{\lambda(k)}=\frac{y}{L t}+i k$ (denoted, again, $\Gamma_{D}$ ), until it strikes the nondegenerate contour defined above for the term $\mathbf{O}_{a}(1) e^{\mu_{3}^{-}(\lambda) x-\int_{K}^{y} \mu_{3}(s ; \lambda) d s}$ (denote here $\Gamma_{N D}$ ). Along $\Gamma_{D}$, we have

$$
\lambda(k)=\frac{y^{2}}{L^{2} t^{2}}+2 i k \frac{y}{L t}-k^{2} ; \quad \operatorname{Re} \lambda(k)^{2}=\frac{y^{4}}{L^{4} t^{4}}-6 \frac{y^{2}}{L^{2} t^{2}} k^{2}+k^{4},
$$

and consequently

$$
\operatorname{Re}\left(\lambda t+\mu_{3}^{-}(\lambda) x-\sqrt{\lambda} y\right) \leq-\frac{y^{2}}{M t}-k^{2}\left(t-\frac{x}{a_{1}^{-}}\right)+\frac{x}{\left(a_{1}^{-}\right)^{3}} k^{4} .
$$

In this case, our contours $\Gamma_{D}$ and $\Gamma_{N D}$ intersect for

$$
k^{2}=\frac{\frac{y^{2}}{L^{2} t^{2}}-\lambda_{R}}{1+\mathbf{O}\left(\frac{y^{2}}{L^{2} t^{2}}\right)} \text {. }
$$

In the case $-t^{-1 / 2} \leq \frac{\tilde{\alpha}}{\tilde{p}} \leq 0$, we have $\lambda_{R}=-t^{-1 / 2}$, and the growth term $\frac{x}{\left(a_{1}^{-}\right)^{3}} k^{4}$ remains bounded. For the second case $-\epsilon \leq \frac{\tilde{\alpha}}{\tilde{p}} \leq-t^{-1 / 2}$, we choose

$$
\lambda_{R}=\frac{\tilde{\alpha}}{\tilde{p}}=\frac{1}{2} \frac{\frac{x}{a_{1}^{-}}-\int_{K}^{y} \frac{d s}{a_{1}(s)}-t}{\frac{x}{\left(a_{1}^{-}\right)^{3}}-\int_{K}^{y} \frac{d s}{a_{1}(s)^{3}}}
$$

for which $\Gamma_{D}$ and $\Gamma_{N D}$ intersect for

$$
k^{2}=-\frac{1}{2} \frac{\frac{x}{a_{1}^{-}}-\int_{K}^{y} \frac{d s}{a_{1}(s)}-t}{\frac{x}{\left(a_{1}^{-}\right)^{3}}-\int_{K}^{y} \frac{d s}{a_{1}(s)^{3}}}+\mathbf{O}\left(\frac{y^{2}}{L^{2} t^{2}}\right)
$$

We have, then,

$$
\begin{aligned}
-\frac{y^{2}}{M t}-k^{2}\left(t-\frac{x}{a_{1}^{-}}\right)+\frac{x}{\left(a_{1}^{-}\right)^{3}} k^{4} & \leq-\frac{y^{2}}{M t}-k^{2} \frac{x}{\left(a_{1}^{-}\right)^{3}}\left(\frac{t-\frac{x}{a_{1}^{-}}}{\frac{x}{\left(a_{1}^{-}\right)^{3}}}-k^{2}\right) \\
& \leq-\frac{y^{2}}{M t}-\frac{1}{2} k^{2}\left(t-\frac{x}{a_{1}^{-}}\right) .
\end{aligned}
$$

Our estimate becomes

$$
\mathbf{O}\left(t^{-1 / 2}\right) \wedge\left[\mathbf{O}\left(\left|x-a_{1}^{-} t\right|^{-1}\right) e^{-\frac{y^{2}}{M t}}\right] I_{\left\{\left|x-\int_{K}^{y} \frac{d s}{a_{1}(s)}\right| \leq\left|a_{1}^{-}\right| t\right\}},
$$

where the time decay $\mathbf{O}\left(t^{-1 / 2}\right)$ can be obtained in the usual way by integrating along essential spectrum. Finally, we observe that for $y>0$

$$
e^{-\sqrt{\lambda} y}=1+\mathbf{O}(|\sqrt{\lambda} y|)
$$

for which we have

$$
\mathbf{O}_{a}(1) e^{\mu_{3}^{-}(\lambda) x-\sqrt{\lambda} y}=\mathbf{O}_{a}(1) e^{\mu_{3}^{-}(\lambda) x}+\mathbf{O}(|\sqrt{\lambda} y|) e^{\mu_{3}^{-}(\lambda) x} .
$$


Proceeding through the nondegenerate analysis with $\mathbf{O}_{a}(1) e^{\mu_{3}^{-}(\lambda) x}$, and proceeding as above for the estimates $\mathbf{O}(|\sqrt{\lambda} y|) e^{\mu_{3}^{-}(\lambda) x}$, we obtain an alternative estimate of

$$
\mathbf{O}\left(t^{-1 / 2}\right) e^{-\frac{\left(x-a_{1}^{-} t\right)^{2}}{M t}}+\left[\mathbf{O}\left(t^{-3 / 4}\right) \wedge\left(\mathbf{O}(|y|) \mathbf{O}\left(\left|x-a_{1}^{-} t\right|^{-3 / 2}\right)\right)\right] .
$$

We next consider the term $\bar{u}_{x}(x) \mathbf{O}_{a}\left(|\lambda|^{-1}\right) e^{-\sqrt{\lambda} y}$ in the case $\frac{\tilde{\alpha}}{\tilde{p}}<0$, for which we take the contour defined through $\sqrt{\lambda(k)}=\frac{y}{L t}+i k$. Following Howard (2002b), we divide the integrand into an analytic piece plus a non-analytic error,

$$
\int_{\Gamma^{*}} \frac{e^{\lambda t-\sqrt{\lambda} y}}{\lambda} d \lambda=\int_{\Gamma^{*}} \frac{e^{\lambda t}}{\lambda} d \lambda+\int_{\Gamma^{*}} \frac{e^{\lambda t}\left(e^{-\sqrt{\lambda} y}-1\right)}{\lambda} d \lambda .
$$

For the first we proceed as in the nondegnerate case by taking a contour that passes to the left of the imaginary axis (and to the right of $\Gamma_{d}$ ). By Cauchy's integral formula, we obtain an estimate by

$$
\bar{u}_{x}(x) I_{\left\{\left|x-\int_{L}^{y} \frac{d s}{a_{1}(s)}\right| \leq\left|a_{1}^{-}\right| t\right\}}+\bar{u}_{x}(x) \mathbf{O}\left(e^{-\eta t}\right) .
$$

For the second, we observe that in the case $|\sqrt{\lambda} y|<1$, we have

$$
\left|\int_{\Gamma^{*}} \frac{e^{\lambda t}\left(e^{-\sqrt{\lambda} y}-1\right)}{\lambda} d \lambda\right|=\left|\int_{\Gamma^{*}} \frac{e^{\lambda t} \mathbf{O}(|\sqrt{\lambda} y|)}{\lambda} d \lambda\right|=\mathbf{O}\left(t^{-1 / 2}\right) \mathbf{O}(|y|),
$$

while for $|\sqrt{\lambda} y| \geq 1$, we have $\left|\frac{1}{\sqrt{\lambda}}\right| \leq|y|$, so that

$$
\left|\int_{\Gamma^{*}} \frac{e^{\lambda t}\left(e^{-\sqrt{\lambda} y}-1\right)}{\lambda} d \lambda\right|=\left|\int_{\Gamma^{*}} \frac{e^{\lambda t}\left(e^{-\sqrt{\lambda} y}-1\right) \mathbf{O}(|y|)}{\sqrt{\lambda}} d \lambda\right|=\mathbf{O}\left(t^{-1 / 2}\right) \mathbf{O}(|y|) .
$$

The final term in this case is $\bar{u}_{x}(x) \mathbf{O}\left(\left|\lambda^{-1 / 2} \ln \lambda\right|\right) e^{-\sqrt{\lambda} y}$, for which we again take the contour defined through $\sqrt{\lambda}=\frac{y}{L t}+i k$ to determine an estimate by

$$
\bar{u}_{x}(x) \mathbf{O}\left(t^{-1 / 2} \ln t\right) e^{-\frac{y^{2}}{L t}} .
$$

Derivative estimates follow similarly.

Case (iv) $y \leq 0 \leq x$. According to Lemma 3.1, we have three integrands to evaluate in the case $y \leq 0 \leq x$, beginning with $\mathbf{O}\left(\left|\lambda^{-1 / 2}\right|\right) \mathbf{O}\left(|x|^{-1}\right) e^{-\sqrt{\lambda} x-\mu_{2}^{-}(\lambda) y}$, where we recall

$$
\mu_{2}^{-}(\lambda)=\frac{a_{2}^{-}-\sqrt{\left(a_{2}^{-}\right)^{2}+4 \lambda}}{2}=-\frac{1}{a_{2}^{-}} \lambda+\frac{1}{\left(a_{2}^{-}\right)^{3}} \lambda^{2}+\mathbf{O}\left(\lambda^{3}\right) .
$$

For $|y| \geq a_{2}^{-} t$, we expect the kernel to propagate to the right with speed $a_{2}^{-}$, remaining to the right of the shock layer. Observing that along the contours employed in Case (i), we have $\operatorname{Re}(-\sqrt{\lambda}) \leq \operatorname{Re} \mu_{2}^{-}(\lambda)$, we conclude that by making this estimate we can obtain the initial bound

$$
\mathbf{O}\left(t^{-1 / 4}\right) \mathbf{O}\left(|x|^{-1}\right) e^{-\frac{\left(x-y-a_{2}^{-} t\right)^{2}}{M t}} I_{\left\{|y| \geq a_{2}^{-} t\right\}}
$$


For $|y|<a_{2}^{-} t$, we expect the kernel to cross the shock layer and begin decaying in $x$ according to the degenerate rate $e^{-\frac{x^{2}}{M t}}$. In this case, we take the contour defined through $\sqrt{\lambda}=\frac{x}{L t}+i k\left(\right.$ where $\left.\lambda(k)=\frac{x^{2}}{L^{2} t^{2}}+2 i k \frac{x}{L t}-k^{2}, \operatorname{Re} \lambda(k)^{2}=\frac{x^{4}}{L^{4} t^{4}}-\frac{6 x^{2}}{L^{2} t^{2}} k^{2}+k^{4}\right)$, where $L$ is assumed sufficiently large so that $|\lambda(k)| \leq r$ prior to striking the nondegenerate contour. Along the degenerate contour, we have

$$
\begin{aligned}
& \left|\mathbf{O}\left(|x|^{-1}\right) \int_{\Gamma^{*}} \mathbf{O}\left(\left|\lambda^{-1 / 2}\right|\right) e^{\lambda t-\sqrt{\lambda} x-\mu_{2}^{-}(\lambda) y} d \lambda\right| \\
& \quad=\mathbf{O}\left(|x|^{-1}\right) \int_{-k^{*}}^{+k^{*}} e^{\frac{x^{2}}{L^{2} t}-k^{2} t-\frac{x^{2}}{L t}+\frac{y}{a_{2}^{2}}\left(\frac{x^{2}}{L^{2} t^{2}}-k^{2}\right)-\frac{y}{(a-)^{3}}\left(\frac{x^{4}}{L^{4} t^{4}}-\frac{6 x^{2}}{L^{2} t^{2}} k^{2}+k^{4}\right)} d k \\
& \quad=\mathbf{O}\left(|x|^{-1}\right) \mathbf{O}\left(\left|y+a_{2}^{-} t\right|^{-1 / 2}\right) e^{-\frac{x^{2}}{L t}} I_{\left\{|y| \leq a_{2}^{-} t\right\}},
\end{aligned}
$$

where as in the analysis of Case (i), our contours $\Gamma_{D}$ and $\Gamma_{N D}$ intersect for $k$ sufficiently small so that the growth terms are subsumed. Finally, we observe that for $|y| \geq a_{2}^{-} t$, we have

$$
\begin{aligned}
t^{-1 / 4} e^{-\frac{\left(x-y-a_{2}^{-} t\right)^{2}}{M t}} & =\frac{\left|x-y-a_{2}^{-} t\right|^{1 / 2}}{t^{1 / 4}}\left|x-y-a_{2}^{-} t\right|^{-1 / 2} e^{-\frac{\left(x-y-a_{2}^{-} t\right)^{2}}{M t}} \\
& \leq C\left|x-y-a_{2}^{-} t\right|^{-1 / 2} e^{-\frac{\left(x-y-a_{2}^{-} t\right)^{2}}{2 M t}} \leq C\left|y+a_{2}^{-} t\right|^{-1 / 2} e^{-\frac{\left(x-y-a_{2}^{-} t\right)^{2}}{M t}}
\end{aligned}
$$

The analysis of our second term from Lemma 3.1,

$$
\mathbf{O}\left(\left|\lambda^{-1 / 2} \ln \lambda\right|\right) \mathbf{O}\left(|x|^{-2}\right) e^{-\sqrt{\lambda} x-\mu_{2}^{-}(\lambda) y},
$$

is almost identical and can be estimated by

$$
\mathbf{O}\left(t^{-1 / 4} \ln t\right) \mathbf{O}\left(|x|^{-2}\right) e^{-\frac{\left(x-y-a_{2}^{-} t\right)^{2}}{M t}} I_{\left\{|y| \leq a_{2}^{-} t\right\}}+\mathbf{O}\left(\left|y+a_{2}^{-} t\right|^{-1 / 2} \ln t\right) e^{-\frac{x^{2}}{M t}} I_{\left\{|y| \leq a_{2}^{-} t\right\}} .
$$

The excited term, $\bar{u}_{x}(x) \mathbf{O}_{a}\left(\left|\lambda^{-1}\right|\right) e^{-\mu_{2}^{-}(\lambda) y}$, can be analyzed as is its counterpart from Case (ii). Derivative estimates follow similarly.

Case (v) $0<K \leq y \leq x$. According to Lemma 3.1, we have seven integrands to evaluate in the case $0<K \leq y \leq x$, beginning with $\mathbf{O}\left(\left|\lambda^{-1 / 2}\right|\right) \mathbf{O}\left(|x|^{-1}\right) \mathbf{O}(|y|) e^{-\sqrt{\lambda}|x-y|}$ and $\mathbf{O}_{a}\left(\left|\lambda^{-1}\right|\right) \mathbf{O}\left(|x|^{-2}\right) e^{-\sqrt{\lambda}|x-y|}$, which were both analyzed in the beginning of this section, with combined estimate

$$
\mathbf{O}\left(t^{-1 / 2}\right) \mathbf{O}\left(|x|^{-1}\right) \mathbf{O}(|y|) e^{-\frac{(x-y)^{2}}{M t}}+P_{+} \bar{u}_{x}(x) I_{\left\{|x-y| \leq \epsilon_{0} \sqrt{t}\right\}} .
$$

The third integrand, $\mathbf{O}\left(\left|\lambda^{-1 / 2}(\ln \lambda)^{2}\right|\right) \mathbf{O}\left(|x|^{-2}\right) \mathbf{O}(|y|) e^{-\sqrt{\lambda}|x-y|}$, can be analyzed similarly to obtain an estimate by $\mathbf{O}\left(t^{-1 / 2}(\ln t)^{2}\right) \mathbf{O}\left(|x|^{-2}\right) \mathbf{O}(|y|) e^{-\frac{(x-y)^{2}}{M t}}$.

We next consider the integrand

$$
\mathbf{O}\left(\left|\lambda^{-1 / 2}\right|\right) \mathbf{O}\left(|x|^{-1}\right) e^{-\sqrt{\lambda} x-\int_{K}^{y} \mu_{3}(s ; \lambda) d s} .
$$

We observe that for $\left|\int_{K}^{y} \frac{d s}{a_{1}(s)}\right| \geq t$, we may use the observation that $\operatorname{Re}(-\sqrt{\lambda} x) \leq$ $\operatorname{Re}\left(-\int_{K}^{x} \mu_{3}(s ; \lambda) d s\right)$ and take a contour as in the nondegenerate analysis. In this way 
we obtain an estimate of the form

$$
\mathbf{O}\left(t^{-1 / 4}\right) \mathbf{O}\left(|x|^{-1}\right) e^{-\frac{\left(\int_{K}^{x} \frac{d s}{a_{1}(s)}+\int_{K}^{y} \frac{d s}{a_{1}(s)}+t\right)^{2}}{M t}} I_{\left\{\left|\int_{K}^{y} \frac{d s}{a_{1}(s)}\right| \geq t\right\}}
$$

In the case $\left|\int_{K}^{y} \frac{d s}{a_{1}(s)}\right|<t$, we take the contour defined through $\sqrt{\lambda(k)}=\frac{x}{L t}+i k$, with

$$
\lambda(k)=\frac{x^{2}}{L^{2} t^{2}}+2 i k \frac{x}{L t}-k^{2} ; \quad \operatorname{Re} \lambda(k)^{2}=\frac{x^{4}}{L^{4} t^{4}}-\frac{6 x^{2}}{L^{2} t^{2}} k^{2}+k^{4}
$$

until it intersects the nondegenerate contour. We have, along $\Gamma_{D}$,

$$
\begin{aligned}
& \left|\mathbf{O}\left(|x|^{-1}\right) \int_{\Gamma^{*}} \mathbf{O}\left(\left|\lambda^{-1 / 2}\right|\right) e^{\lambda t-\sqrt{\lambda} x-\int_{K}^{y} \mu_{3}(s ; \lambda) d s} d \lambda\right| \\
& =\left|\mathbf{O}\left(|x|^{-1}\right) \int_{\Gamma^{*}} \mathbf{O}\left(\left|\lambda^{-1 / 2}\right|\right) e^{\lambda t-\sqrt{\lambda} x+\lambda \int_{K}^{y} \frac{d s}{a_{1}(s)}-\lambda^{2} \int_{K}^{y} \frac{d s}{a_{1}(s)^{2}}+\mathbf{O}\left(|\lambda|^{3}\right)} d \lambda\right| \\
& =\mathbf{O}\left(|x|^{-1}\right) \int_{-k^{*}}^{+k^{*}} e^{\frac{x^{2}}{L^{2} t}-k^{2} t-\frac{x^{2}}{L t}+\left(\frac{x^{2}}{L^{2} t^{2}}-k^{2}\right) \int_{K}^{y} \frac{d s}{a_{1}(s)}-\left(\frac{x^{4}}{L^{4} t^{4}}-\frac{6 x^{2}}{L^{2} t^{2}} k^{2}+k^{4}\right) \int_{K}^{y} \frac{d s}{a_{1}(s)^{3}} d s+\mathbf{O}\left(|\lambda(k)|^{3}\right)} d k \\
& =\mathbf{O}\left(\left|\int_{K}^{y} \frac{d s}{a_{1}(s)}+t\right|^{-1 / 2}\right) \mathbf{O}\left(|x|^{-1}\right) e^{-\frac{x^{2}}{M_{t}}} I_{\left\{\left|\int_{K}^{y} \frac{d s}{a_{1}(s)}\right| \leq t\right\}},
\end{aligned}
$$

which we observe can be subsumed (in the case $x \geq y \geq 0$ ) into previous estimates.

Similarly, the summand $\mathbf{O}\left(\left|\lambda^{-1 / 2} \ln \lambda\right|\right) \mathbf{O}\left(|x|^{-2}\right) e^{-\sqrt{\lambda} x-\int_{K}^{y} \mu_{3}(s ; \lambda) d s}$ leads to an estimate by

$$
\begin{aligned}
& \mathbf{O}\left(t^{-1 / 4} \ln t\right) \mathbf{O}\left(|x|^{-2}\right) e^{-\frac{\left(\int_{K}^{x} \frac{d s}{a_{1}(s)}+f_{K}^{y} \frac{d s}{a_{1}(s)}+t\right)^{2}}{M t}} I_{\left\{\left|\int_{K}^{y} \frac{d s}{a_{1}(s)}\right| \geq t\right\}} \\
& +\mathbf{O}\left(\left|\int_{K}^{y} \frac{d s}{a_{1}(s)}+t\right| \ln t\right) \mathbf{O}\left(|x|^{-2}\right) e^{-\frac{x^{2}}{M_{t}}} I_{\left\{\left|\int_{K}^{y} \frac{d s}{a_{1}(s)}\right| \leq t\right\}},
\end{aligned}
$$

the latter of which can be subsumed into previous estimates.

The summand $\bar{u}_{x}(x) \mathbf{O}_{a}\left(\left|\lambda^{-1}\right|\right) e^{-\int_{K}^{y} \mu_{3}(s ; \lambda) d s}$, has been evaluated in Case (iii), and the summand

$$
\bar{u}_{x}(x) \mathbf{O}_{a}\left(\left|\lambda^{-1}\right|\right) e^{-\int_{K}^{y} \mu_{3}(s ; \lambda) d s}
$$

can be analyzed by nondegenerate methods to obtain an exponentially decaying excited term plus further estimates, that due to their exponential rate of decay in $x$ can be subsumed. The integrand $\mathbf{O}_{a}(1) e^{\int_{y}^{x} \mu_{1}(s ; \lambda) d s}$ can also be treated by nondegenerate methods, which lead to an estimate that can be subsumed.

Case (vi) $0<K \leq x \leq y$. According to Lemma 3.1, we have eight integrals to evaluate in the case $0<K \leq x \leq y$, beginning with the summands

$$
\mathbf{O}\left(\left|\lambda^{-1 / 2}\right|\right) \mathbf{O}\left(|x|^{-1}\right) \mathbf{O}(|y|) e^{-\sqrt{\lambda}|x-y|} \quad \text { and } \quad \mathbf{O}_{a}\left(\left|\lambda^{-1}\right|\right) \mathbf{O}\left(|x|^{-2}\right) e^{-\sqrt{\lambda}|x-y|} \text {, }
$$


which were analyzed in the introduction of this section. Following the arguments introduced there, we also find that the summand $\mathbf{O}\left(\left|\lambda^{-1 / 2}(\ln \lambda)^{2}\right|\right) \mathbf{O}\left(|x|^{-1}\right) e^{-\sqrt{\lambda}|x-y|}$ leads to an estimate by

$$
\mathbf{O}\left(t^{-1 / 2}(\ln t)^{2}\right) \mathbf{O}\left(|x|^{-1}\right) e^{-\frac{(x-y)^{2}}{M t}}
$$

For the summand $\mathbf{O}_{a}(1) e^{-\int_{x}^{y} \mu_{3}(s ; \lambda) d s}, x \geq K$, we can proceed by taking contours from the non-degenerate case to obtain an estimate by

$$
\mathbf{O}\left(t^{-1 / 2}\right) e^{-\frac{\left(\int_{x}^{y} \frac{d s}{a_{1}(s)}+t\right)^{2}}{M t}}
$$

For the summand $e^{-\int_{x}^{y} \mu_{3}(s ; \lambda) d s} \mathbf{O}\left(\left|\lambda^{1 / 2} \ln \lambda\right|\right)$, we first consider the case $\left|\int_{y}^{x} \frac{d s}{a_{1}(s)}\right| \geq t$, for which the nondegenerate contours do not cross the negative real axis, and taking them, we conclude an estimate of $\mathbf{O}\left(t^{-3 / 4} \ln t\right) e^{-\frac{\left(\int_{y}^{x} \frac{d s}{a_{1}(s)}+t\right)^{2}}{M t}}$. For the case $\left|\int_{x}^{y} \frac{d s}{a_{1}(s)}\right|<t$, we proceed as in the analysis of the summand $\mathbf{O}\left(\left|\lambda^{1 / 2} \ln \lambda\right|\right) e^{\mu_{3}^{-}(\lambda) x-\mu_{2}^{-}(\lambda) y}$ in Case (i), and take the contour defined through $\sqrt{\lambda}=t^{-1}+i k$ until it strikes the nondegenerate contour defined through

$$
-\lambda \int_{x}^{y} \frac{d s}{a_{1}(s)}+\lambda^{2} \int_{x}^{y} \frac{d s}{a_{1}(s)^{3}}=-\lambda_{R} \int_{x}^{y} \frac{d s}{a_{1}(s)}+\lambda_{R}^{2} \int_{x}^{y} \frac{d s}{a_{1}(s)^{3}}+i l \int_{x}^{y} \frac{d s}{a_{1}(s)},
$$

with $\lambda_{R}$ chosen according to

$$
\lambda_{R}= \begin{cases}-\epsilon, & -\epsilon \geq \frac{\bar{\alpha}_{+}}{\bar{p}_{+}}, \\ \frac{\bar{\alpha}_{+}}{\bar{p}_{+}}, & -t^{-1 / 2} \geq \frac{\bar{\alpha}_{+}}{\bar{p}_{+}} \geq-\epsilon \\ -t^{-1 / 2}, & 0 \geq \frac{\bar{\alpha}_{+}}{\bar{p}_{+}} \geq-t^{-1 / 2}\end{cases}
$$

where

$$
\lambda_{R}=\frac{\bar{\alpha}_{+}}{\bar{p}_{+}} ; \quad \bar{\alpha}_{+}:=\frac{\int_{x}^{y} \frac{d s}{a_{1}(s)}+t}{2 t} ; \quad \bar{p}_{+}:=\int_{x}^{y} \frac{d s}{a_{1}(s)^{3}} \leq 0 .
$$

We determine an estimate by

$$
\mathbf{O}\left(t^{-3 / 4} \ln t\right) \wedge \mathbf{O}\left(\left|t+\int_{x}^{y} \frac{d s}{a_{1}(s)}\right|^{-3 / 2} \ln t\right)
$$

which for $t$ bounded away from zero can alternatively be written in the form

$$
\mathbf{O}\left(\left(1+\left|t+\int_{x}^{y} \frac{d s}{a_{1}(s)}\right|+t^{1 / 2}\right) \ln (e+t)\right) .
$$

The remaining cases were analyzed in Case (v). 


\section{References}

Alexander, J., Gardner, R., Jones, C. K. R. T. (1990). A topological invariant arising in the analysis of traveling waves. J. Reine Angew. Math. 410:167-212.

Brin, L. (2001). Numerical testing of the stability of viscous shock waves. Math. Cont. 70(235):1071-1088.

Dodd, J. (1996). Convection stability of shock profile solutions of a modified KdV-Burgers equation. Ph.D. thesis under the direction of R. L. Pego, University of Maryland.

Evans, J. W. (1972/1975). Nerve Axon Equations I-IV. Indiana. Univ. Math. J. 21:877-885; 22:75-90; 22:577-594; 24:1169-1190.

Freistuhler, H., Szmolyan, P. (2002). Spectral stability of small shock waves. Arch. Rational Mech. Anal. 164:287-309.

Gardner, R., Zumbrun, K. (1998). The Gap Lemma and geometric criteria for instability of viscous shock profiles. Comm. Pure Appl. Math. 51:789-847.

Goodman, J. (1986). Nonlinear asymptotic stability of viscous shock profiles for conservation laws. Arch. Rational Mech. Anal. 95(4):325-344.

Howard, P. (2002a). Pointwise estimates and stability for degenerate viscous shock waves. J. Reine Angew. Math. 545:19-65.

Howard, P. (2002b). Local tracking and stability for degenerate viscous shock waves. J. Differential Equations 186:440-469.

Howard, P. Nonlinear stability of degenerate shock profiles. Preprint 2005.

Howard, P., Zumbrun, K. (2000). Pointwise estimates and stability for dispersive-diffusive shock waves. Arch. Rational Mech. Anal.155:85-169.

Humpherys, J., Zumbrun, K. (2002). Spectral stability of small amplitude shock profiles for dissipative symmetric hyperbolic-parabolic systems. Z. Angew. Math. Phys. 53: 20-34.

Howard, P., Zumbrun, K. (2004). The Evans function and stability criteria for degenerate viscous shock waves. Discrete and Continuous Dynamical Systems 10:837-855.

Jones, C. K. R. T. (1984). Stability of the traveling wave solution of the FitzHugh-Nagumo system. Trans. Amer. Math. Soc. 286(2):431-469.

Kapitula, T., Sandstede, B. (1998). Stability of bright solitary-wave solutions to perturbed nonlinear Schrodinger equations. Physica D 124:58-103.

Kawashima, S., Matsumura, A. (1985). Asymptotic stability of traveling wave solutions of systems for one-dimensional gas motion. Comm. Math. Phys. 101(1):97-127.

Kawashima, S., Matsumura, A., Nishihara, K. (1986). Asymptotic behavior of solutions for the equations of a viscous heat-conductive gas. Proc. Japan. Acad. Ser. A Math. Sci. 62(7):249-252.

Matsumura, A., Nishihara, K. (1985). On the stability of travelling waves solutions of a one-dimensional model system for compressible viscous gas. Japan. J. Appl. Math. 2(1):17-25.

Nishihara, K. (1995). Stability of traveling waves with degenerate shock for systems of a one-dimensional viscoelastic model. J. Differential Equations 120:304-318.

Oh, M., Zumbrun, K. (2003). Stability of periodic solutions of viscous conservation laws: Analysis of the Evans function. Arch. Rational Mech. Anal. 166:167-196.

Pego, R. L., Weinstein, M. I. (1992). Eigenvalues and instabilities of solitary waves. Philos. Trans. Roy. Soc. London Ser. A 340:47-94.

Plaza, R., Zumbrun, K. (2004). An Evans function approach to spectral stability of smallamplitude viscous shock profiles. Discrete Contin. Dyn. Syst. 10(4):885-924.

Sandstede, B., Scheel, A. (2004). Evans function and blow-up methods in critical eigenvalue problems. Discrete Contin. Dyn. Syst. 10(4):941-964.

Zumbrun, K., Howard, P. (1998/2002). Pointwise semigroup methods and stability of viscous shock waves. Indiana U. Math. J. 47(3):741-871. Also, Errata. Indiana U. Math. J. 51(4):1017-1021. 